\title{
Investigation of Neuronal Membrane Fusion Using Fluorescence Correlation Spectroscopy
}

\author{
Dissertation \\ for the award of the degree \\ "Doctor rerum naturalium" \\ of the Georg August University Göttingen, \\ submitted by
}

Wensi Vennekate

From Tongliaoshi, China

Göttingen 2012 
Members of the Thesis Committee:

\section{Prof. Dr. Peter Jomo Walla (Referee)}

Workgroup Biomolecular Spectroscopy and Single Molecule Detection Max Planck Institute for biophysical Chemistry, Göttingen and

Department Biophysical Chemistry

Institute for physical and theoretical Chemistry

Technische Universität Carolo-Wilhelmina zu Braunschweig

\section{Prof. Dr. Reinhard Jahn (Referee)}

Department Neurobiology

Max Planck Institute for biophysical Chemistry, Göttingen

\section{Prof. Dr. Claudia Steinem}

Institute for Organic and Biomolecular Chemistry

Georg August University, Göttingen

Date of oral examination: November 8th 2012. 
I hereby declare that the Ph.D. thesis entitled "Investigation of Neuronal Membrane Fusion using Fluorescence Correlation Spectroscopy" has been written independently and with no other sources and aids than quoted.

Göttingen, September 30th, 2012. 


\section{Contents}

\begin{tabular}{lll}
\hline Introduction & 1
\end{tabular}

1.1 Neuronal exocytosis $\ldots \ldots \ldots \ldots \ldots$. . . . . . . . . . . . . . .

1.2 The role of SNARE proteins in exocytosis $\ldots \ldots \ldots \ldots$

1.3 Calcium sensor protein: synaptotagmin-1 . . . . . . . . . . . . . 6

1.4 NSF and $\alpha$-SNAP $\ldots \ldots \ldots \ldots \ldots$

1.5 Fluorescence correlation spectroscopy $\ldots \ldots$. . . . . . . . . . . . 11

$1.5 .1 \quad$ Fluorescence correlation spectroscopy (FCS) . . . . . . . . . . . 11

1.5.2 Fluorescence cross-correlation spectroscopy (FCCS) . . . . . . . . 14

1.5.3 Two-photon excitation (TPE) . . . . . . . . . . . . . . . . 15

1.5.4 Förster resonance energy transfer (FRET) . . . . . . . . . . . . . 17

1.6 Outline of this Study $\ldots \ldots \ldots \ldots \ldots \ldots$

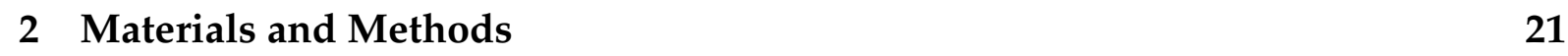

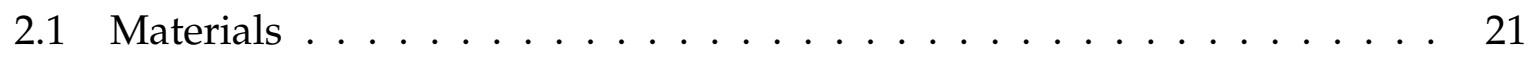

2.1 .1 Chemicals . . . . . . . . . . . . . . . . . 21

2.1 .2 Phospholipids . . . . . . . . . . . . . . . . . 21

2.1 .3 Proteins . . . . . . . . . . . . . . . . . . 22

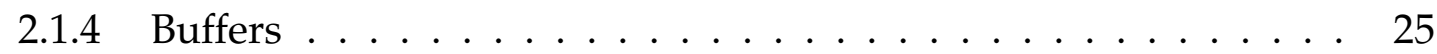


2.1 .5 Instruments, filters, columns and others . . . . . . . . . . . . . 29

2.1 .6 Software . . . . . . . . . . . . . . . . . . 30

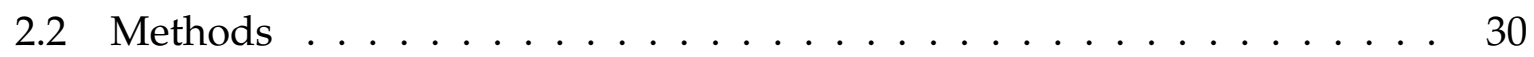

2.2.1 Expression and purification of synaptotagmin-1 . . . . . . . . . 30

2.2 .2 Determination of the protein concentration . . . . . . . . . 34

2.2.3 Determination of Rhodamine Green concentration with UV absorption spectroscopy $\ldots \ldots \ldots$. . . . . . . . . . . 35

2.2 .4 SDS-PAGE . . . . . . . . . . . . . . . . . 36

2.2 .5 Reconstitution of liposomes . . . . . . . . . . . . . . 37

2.2 .6 Purification of mouse and rat SVs $\ldots \ldots \ldots$. . . . . . . . 38

2.2 .7 Purification of chromaffin granules (CGs) . . . . . . . . . . . . . . 39

$2.2 .8 \quad$ Two-photon confocal fluorescence microscopy setup . . . . . . . 39

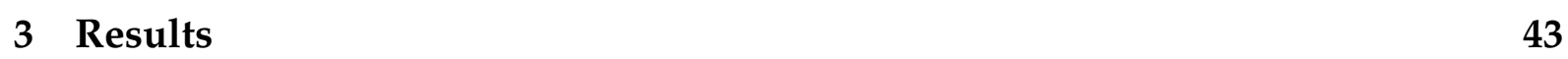

3.1 Cis- and trans-membrane interaction of synaptotagmin-1 . . . . . . . . . 43

3.1.1 Tethering assay based on FCS and FCCS . . . . . . . . . . . 44

3.1.2 Cis- and trans-interaction of Syt1 to the acidic lipid membrane . . 46

3.1.3 The soluble C2AB domain of Syt1 is able to cluster liposomes at a saturating concentration . . . . . . . . . . . . 50

3.1.4 Syt1-SNARE interaction mediated membrane tethering . . . . . . 54

$3.2 \quad$ Characterization of mouse synaptic vesicles by average protein mass . . 57

$3.2 .1 \quad$ Synaptic vesicle labeling with FM 1-43 dye . . . . . . . . . . . . . 59

3.2 .2 Concentration determination of SVs using TP-FCS. . . . . . . . . 59

3.2.3 Background correction due to the dark FM fluorescence . . . . . 61 
3.2.4 Comparing the mass of proteins per vesicle of mouse and rat SVs 62

$3.3 \quad \alpha$-SNAP inhibits SNARE-mediated membrane fusion but not partial SNARE-zippering . . . . . . . . . . . . . . . . . 64

3.3.1 Determination of CG docking by observing variations in diffusion time . . . . . . . . . . . . . . . . . . 67

$3.3 .2 \alpha$-SNAP does not fully block CG docking $\ldots \ldots \ldots$

\begin{tabular}{lll}
\hline 4 & Discussion & 73 \\
\hline
\end{tabular}

$4.1 \quad$ The conflict of cis- and trans-membrane interaction of synaptotagmin-1 . 73

$4.2 \alpha$-SNAP inhibits SNARE-mediated CG fusion, but does not fully block CG docking . . . . . . . . . . . . . . . . 78

4.3 Quantitative characterization of synaptic vesicles . . . . . . . . . . . . . 80

$\begin{array}{lll}5 & \text { Conclusions } & 81\end{array}$

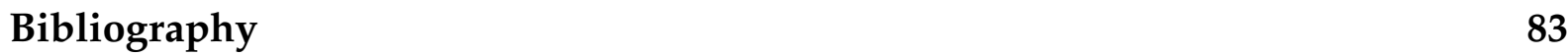

\begin{tabular}{ll}
\hline List of Figures & 101
\end{tabular}

\begin{tabular}{ll}
\hline List of Tables & 105
\end{tabular}

\begin{tabular}{|l|l|l|l|l}
\hline Abbreviations and Symbols & 107
\end{tabular}

\begin{tabular}{ll}
\hline Publication & 111
\end{tabular} 


\section{Chapter 1}

\section{Introduction}

\subsection{Neuronal exocytosis}

Neuronal communication requires fast exocytosis of synaptic vesicles. Synaptic vesicles (SVs) possess a large variety of trafficking proteins in different copy numbers and are filled with neurotransmitters in the lumen [119]. SVs function as transporters of neurotransmitters and release their neurotransmitters by rapid fusion with the presynaptic plasma membrane triggered by calcium influx upon arrival of an action potential [62, 63, 139].

Filled SVs translocate to the active zone, in which SVs target the plasma membrane (docking) and prepare themselves for fusion (priming). The priming state involves protein-protein interaction as well as protein-lipid interaction (priming). Subsequently, they fuse with the plasma membrane initiated by the local increase of the intracellular calcium concentration (fusion, see Figure 1.1] [62, 113, 117].

After fusion, new empty SVs are reformed from the plasma membrane by clathrin coated vesicle fission, a process termed endocytosis [45]. The new SVs are recycled by fusion with the early endosome and regenerated by budding from the early endosome [113]. The regenerated SVs are refilled with neurotransmitters triggered by an electrochemical gradient (NT uptake) and proceed to a new round of the exo- and 
endocytosis (Figure 1.1) [113].

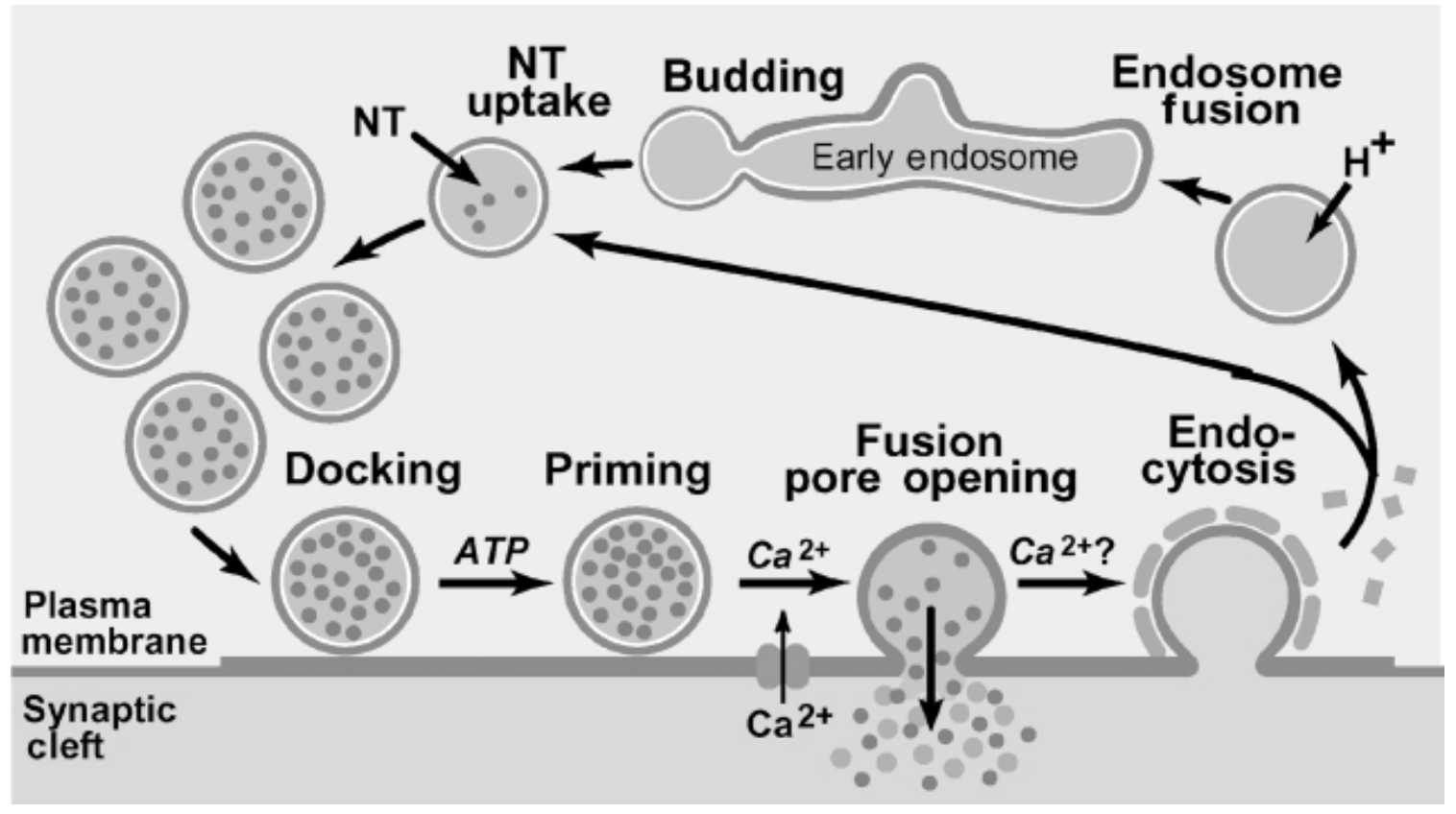

Figure 1.1: Synaptic vesicle cycle [113]. Neurotransmission is mediated by fusion of synaptic vesicles with the plasma membrane. During this process, neurotransmitters are released from the vesicle lumen into the synaptic cleft. After fusion, new SVs are reformed from the plasma membrane by endocytosis. Empty vesicles are recycled from the early endosome by endosome fusion and regenerated by budding from the early endosome. The vesicles are then filled with neurotransmitters (NT uptake) and are available for neurotransmission. Before fusion, the vesicles dock to the plasma membrane and proceed to the priming state, at which point the vesicles are ready for fusion. The fusion occurs when the calcium channels are opened upon arrival of an action potential.

\subsection{The role of SNARE proteins in exocytosis}

Neuronal exocytosis is mediated and regulated by a series of proteins, among which SNARE (soluble N-ethylmaleimide-sensitive-factor attachment receptor) proteins play a central role. The formation of a ternary complex of SNARE proteins is 
crucial for the overall process and the SNARE complex itself is the minimal machinery of neuronal membrane fusion [17, 20, 62, 102, 138]. In neuronal exocytosis, the SNARE complex consists of synaptobrevin 2 (Sb2), syntaxin 1A (Sx1A) and synaptosomalassociated protein 25 (SNAP25).

$\mathrm{Sb} 2$ is a vesicular SNARE protein [119], whereas Sx1A and SNAP25 are located in the plasma membrane and form a complex [34,38]. The core of a SNARE complex is a bundle of four $\alpha$-helices, each of them being a so-called SNARE motive [114]. Sb2 and Sx1A each provide one motif and SNAP25 contributes two (Figure 1.2).

The SNARE complex has a highly conserved layer structure; each of the layers consists of four amino acid side chains which interact with each other. Due to the composition of the layer structure, SNARE proteins are also classified as QSNAREs (Sx (Qa) and SNAP25 (Qb and Qc)) and R-SNARE (Sb, see Figure 1.3) [36]. The SNARE assembly proceeds from the cytosolic N-terminus to the membrane anchored C-terminus (zippering hypothesis) [48, 91].

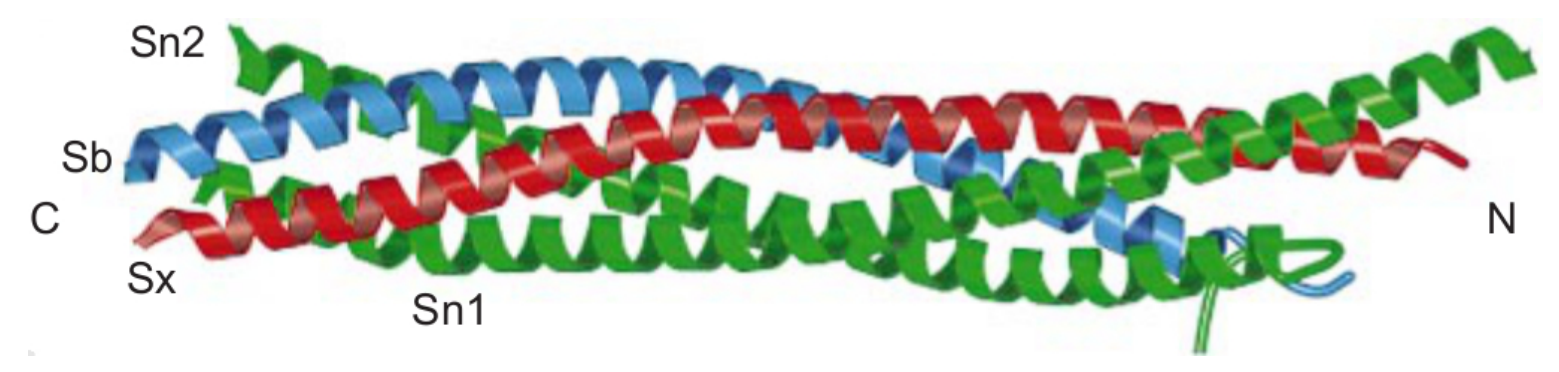

Figure 1.2: Four-helix-bundle of the core SNARE complex [114]. Sb: Synaptobrevin (blue). Sx: Syntaxin (red). Sn1: SNAP-25(N) (green). Sn2: SNAP-25(C) (green).

At the stage of the docked intermediate, SNARE proteins form the trans-complex, in which the C-terminal transmembrane domains of the proteins are in separate membranes. During fusion, the trans-complex converts into the cis-complex form, in which both transmembrane domains of $\mathrm{Sx} 1 \mathrm{~A}$ and $\mathrm{Sb} 2$ come together in the same membrane, and stimulates membrane merging [63]. The cis-complex is inactive for the further membrane fusion and can be disassembled by AAA+ ATPase protein NSF (Nethylmaleimide-sensitive factor) [77] and its cofactor SNAP proteins (soluble NSF at- 


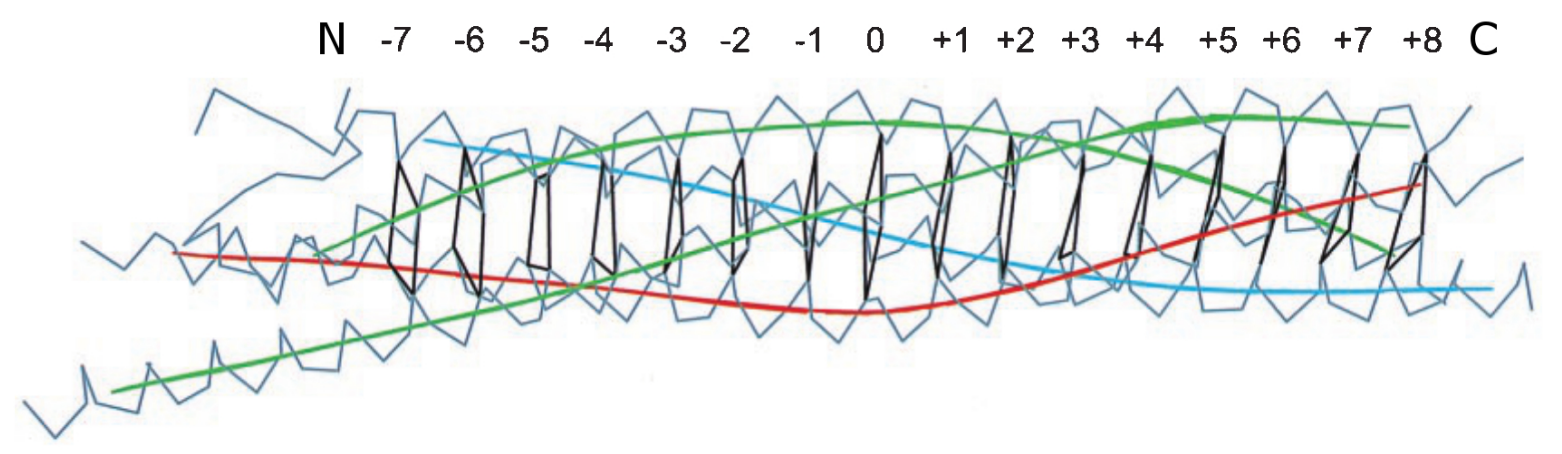

Figure 1.3: Layer structure of the ternary SNARE complex [36]. Synaptobrevin (blue), Syntaxin (red) and SNAP-25 (green) assemble a helix-bundle with a conserved layer structures, in which the +8 layer is the last layer before the C-terminal linker region and the +7 layer is the first layer at the $\mathrm{N}$-termini.

tachment proteins) [22] under ATP hydrolysis.

The SNARE disassembly will be discussed later in Section 1.4. The disassembled Sx and SNAP25 furthermore form the acceptor complex upon invocation by SM (Sec1/Munc-18) family proteins and are available for the further vesicle docking and fusion (Figure 1.4) [63]. SM proteins are not included in this study. 


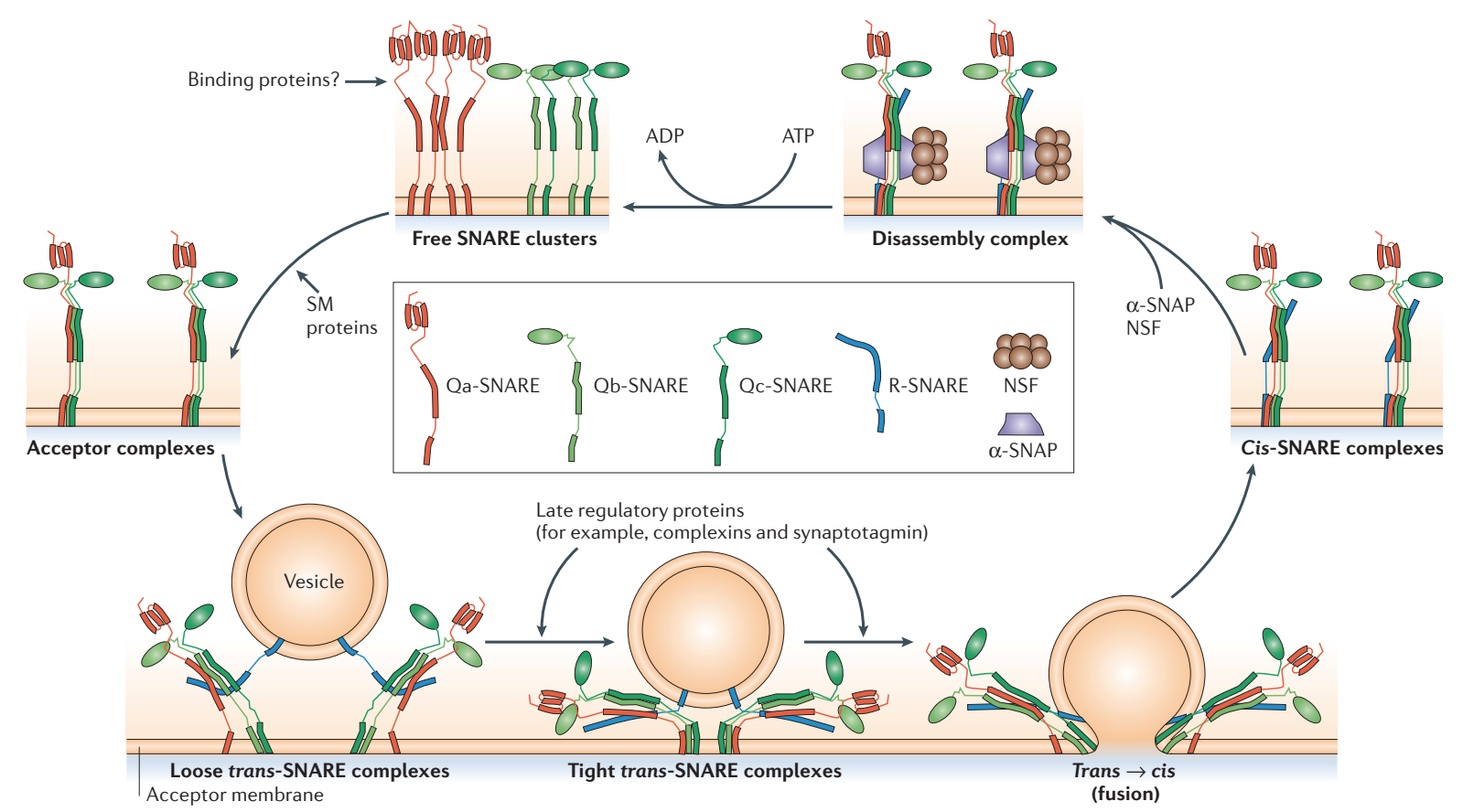

Figure 1.4: The SNARE conformational cycle during vesicle docking and fusion [63]. Three Q-SNAREs (Qa, Qb and Qc) localized in the plasma membrane and one R-SNARE localized in the synaptic vesicles form a fusion machinery. Vesicles are docked to the plasma membrane by SNARE interaction, forming a loose trans-SNARE complex. At the priming stage associated with other late regulatory proteins, SNAREs assemble to a tight trans-complex and are prepared for fusion. Upon arrival of an action potential, SNAREs fully assemble to the cis-conformation and promote membrane fusion. The cis-SNARE-complex is not active for fusion and is disassembled by AAA+ ATPase protein NSF with its cofactor $\alpha$-SNAP under ATP hydrolysis. The disassembled Q-SNAREs are transformed into the active acceptor complex by SM proteins (Sec1/Munc18-related proteins) and can further take part in membrane fusion. 


\subsection{Calcium sensor protein: synaptotagmin-1}

Neuronal exocytosis is triggered by the influx of calcium: Synaptic vesicles are docked at the plasma membrane but do not undergo fusion until an action potential causes a transient increase in the intracellular calcium concentration. The calcium influx is sensed by synaptotagmin-1 (Syt1, $65 \mathrm{kDa}$ ) [19, 37, 79], which is linked to the synaptic vesicle by their transmembrane domains (aa 58-79) in about seven copies [119]. Syt1 possesses two calcium binding C2 domains, referred to as C2A (aa 140-265) and C2B (aa 271-421), which are connected to the transmembrane domain by a long (61 residue) flexible linker [37]. C2A binds three and C2B two calcium ions (Figure 1.5] [37, 95]. Syt1 binds to the acidic phospholipids of the membrane in the presence of calcium [3, 13, 42, 56, 59, 73, 95]. C2B contains a polybasic lysine patch (aa 324-327) proximal to the calcium binding site and has a specific affinity to phosphatidylinositol-4,5-bisphosphate (PiP2) in a calcium independent manner (Figure 1.5) [6, 73, 104, 134, 141]. The polybasic stretch also binds phosphatidylserine (PS). Moreover, Syt1 interacts with syntaxin and SNARE-complex-containing syntaxin in the absence of calcium [6, 19, 21, 69, 125], albeit the binding is influenced to a limited degree by calcium [19,101].

Syt1 is well known as capable to increase the rate of exocytosis by several orders of magnitude [96], but the molecular mechanism is still unclear. Two different priming models are presently being discussed. In the first model, a partial SNARE assembly is formed and arrested at this state, which is assumed to be caused by either an energy barrier or interaction with complexin or synaptotagmin. After receiving calcium, Syt1 may (i) bind to the SNARE complex and the plasma membrane (PiP2, PS), displace the inhibitory complexin and promote the full SNARE zippering [111, 134, 135]; or (ii) bind to the plasma membrane, induce curvature stress close to the membrane contact area and lower the energy barrier [61,78, 82]; (iii) alternatively cross-link the vesicle membrane and the plasma membrane and promote fusion by compensating the membrane charge [3]. The second model proposes tethering/docking of the vesicle to the plasma membrane by Syt1 trans-binding to PiP2 contained in the plasma membrane 
without SNARE assembly. Calcium activates both $\mathrm{C} 2$ domains and thus cross-linking of the membrane to further promote the SNARE assembly. Hereby, Syt1 functions as distance regulator [111, 141].

To dissect the molecular mechanism of Syt1, SNARE-mediated fusion was performed using reconstituted liposomes. Contradictory effects of Syt1 have been reported. Several studies have shown that tethering/docking of Syt1 to the plasma membrane promotes SNARE assembly [61,78]. However, tethering was not observed as a separate intermediate because of the presence of the SNARE proteins. Inhibition of fusion has also been observed in several fusion experiments with SNAREs and Syt1 [67, 111]. This led to further complications in the study, because Syt1 may also bind in cis to the vesicular membrane and cis-binding competes with trans-binding. Since trans-binding of Syt1 is required by all of the different fusion models, a balance between cis- and trans-binding may play an essential role in neurotransmission. Therefore, this study will focus on the cis- and trans-membrane interaction of Syt1. 


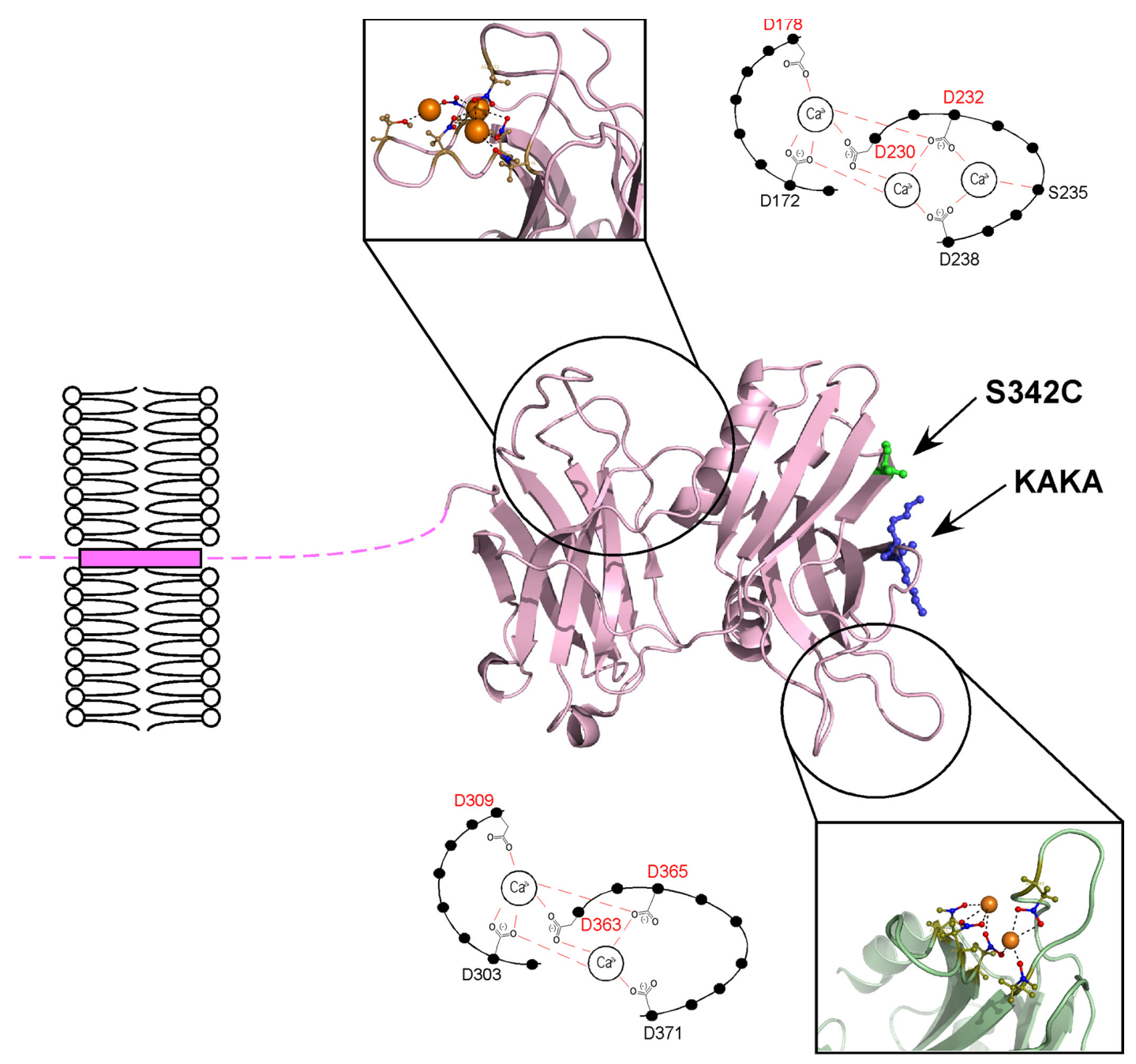

Figure 1.5: 3D structure of synaptotagmin-1. [95] Synaptotagmin-1 possesses one transmembrane domain and two C2 domains, called C2A and C2B. All the three domains are linked subsequently to each other by two flexible linkers. The C2A domain binds three calcium ions, while the C2B domain binds two calcium ions. $\mathrm{Mu}-$ tant $(\mathrm{K} 326,327 \mathrm{~A})$ of the calcium independent binding site of $\mathrm{C} 2 \mathrm{~B}$ is shown as "KAKA" (blue). S342C shows the position of the cysteine mutant, at which the fluorescence dye can be attached (green). 


\subsection{NSF and $\alpha$-SNAP}

NSF (N-ethylmaleimide-sensitive factor) belongs to the AAA+ ATPase family and its function in neuronal exocytosis is to accomplish disassembly of the SNARE complex under ATP hydrolysis [81, 115]. NSF consists of two AAA+ domains, referred to as D1 domain (aa 206-477) and C-terminus D2 domains (aa 478-744), and one N-terminus domain (aa 1-205) [118, 137]. For disassembly, NSF forms a homohexamer [39, 40, 49] and provides the energy for disassembly by its D1 domain, while the C-terminus D2 domain is mainly responsible for the hexamerization [84].

NSF does not bind to the SNARE complex directly. To transfer the energy from the ATP hydrolysis to the SNARE complex, NSF must interact with its cofactor $\alpha$ SNAP (soluble NSF attachment proteins) [22], which consists of fourteen subsequent $\alpha$-helices [80,132] and connects the SNARE complex and the NSF hexamer with two more copies of itself [131]. Altogether, NSF hexamer, three $\alpha$-SNAPs and one SNARE complex form an complex, called $20 S$ complex or 20S particle [40, 49, 57, 131]. The $\mathrm{N}$-termini of the NSF hexamer attach to the $\alpha$-SNAPs at their C-termini [8, 84, 136] and energy is transferred to the SNARE complex via interaction of the N-terminus of $\alpha$-SNAP with the C-terminal side of SNARE complex near the membrane [52]. To process the SNARE disassembly, $\alpha$-SNAP has to attach to the membrane through the hydrophobic loop located at its N-terminus (Figure 1.6) [132]. The disassembly is promoted by the conformation change of the NSF hexamer concerted with the ATP hydrolysis. This movement is transferred to the SNARE complex and the membrane by $\alpha$-SNAPs [49, 115].

Disassembly of the SNARE complex increases the number of free SNARE proteins, and thus plays a positive role for vesicle priming [18, 66, 120, 133]. On the other hand, free $\alpha$-SNAP has been shown to inhibit the vesicle fusion in Drosophila [4] and the exocytosis of dense-core vesicles in PC12 cells [9]. However, how this inhibition of membrane fusion occurs is remaining unclear. 


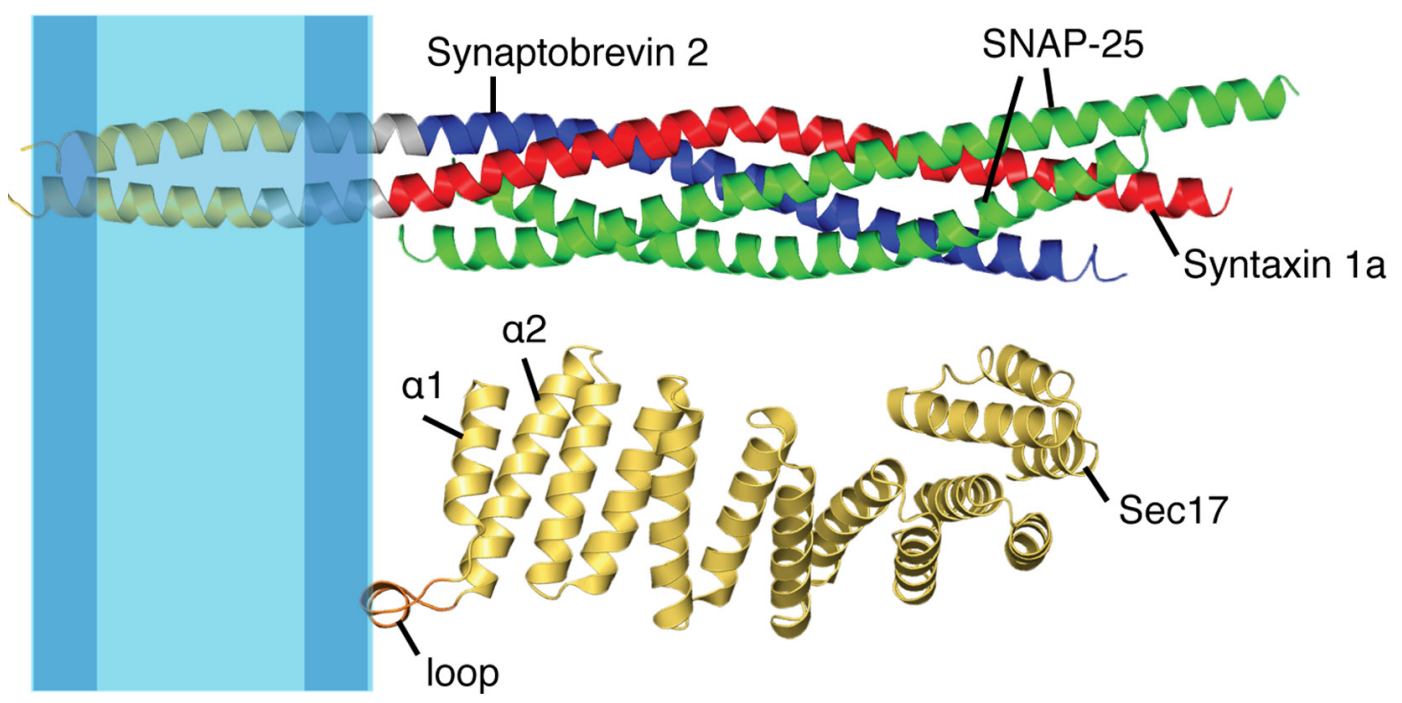

Figure 1.6: Membrane attachment site of $\alpha$-SNAP in the presence of the SNARE complex [132]. The yeast Sec17 ( $\alpha$-SNAP) from Baker's yeast is structured as fourteen subsequent $\alpha$-helices $(\alpha 1-\alpha 14)$ and consists of a hydrophobic loop localized between the $\alpha 1$ - and $\alpha 2$-helices. The loop attaches to the membrane when $\alpha$-SNAP binds the SNARE complex (Synaptobrevin 2, Syntaxin 1a and SNAP-25), and facilitates the disassembly of the SNARE complex by NSF. 


\subsection{Fluorescence correlation spectroscopy}

\subsubsection{Fluorescence correlation spectroscopy (FCS)}

Fluorescence correlation spectroscopy (FCS) is a technique with single molecule level resolution, which is used in this study to temporally characterize the molecular dynamics of biomolecules in vitro in terms of concentration, diffusion time, and interaction with other biomolecular components [5, 31, 76, 98].

The core of FCS is not the characterization of the individual fluorescence event itself-as in time-correlated single photon counting (TCSPC)-but rather the temporal fluctuation of the total fluorescence intensity $I(t)[31,74,75]$ caused by rotational- or translational diffusion [29,75], population of the triplet state [14,25, 130], chemical reactions or interactions [46, 47, 50, 93], Förster Resonance Energy Transfer (FRET) [121, 129]. The fluctuation of a single molecule's emission can be observed only when the sample is strongly diluted, so that the diffusion of a single particle into (on) and out of (off) the effective volume cannot be neglected. The ideal sample concentration for FCS measurements is from subnanomolar to submicromolar.

To analyze experimental data, the photons collected by the detector are added up in time intervals of $5 \mu$ s each and a temporal fluorescence trace $I(t)$ is constructed from these intervals. The fluctuation can be described as [31, 50]:

$$
\delta I(t)=I(t)-\langle I(t)\rangle
$$

where the $\langle I(t)\rangle$ is the mean fluorescence intensity $I(t)$ over the entire time of the experiment $(0-T)$. Autocorrelation is a characterization of data or functions in terms of the "self-similarity", in this case of the signal $I$ at time $t, I(t)$, compared to the signal $I$ at a time shift $t+\tau, I(t+\tau)$ (Figure 1.7). The autocorrelation is computed as [31, 50]:

$$
G(\tau)=\frac{\langle I(t) \cdot I(t+\tau)\rangle}{\langle I(t)\rangle^{2}}-1=\frac{\langle\delta I(t) \cdot \delta I(t+\tau)\rangle}{\langle I(t)\rangle^{2}}
$$




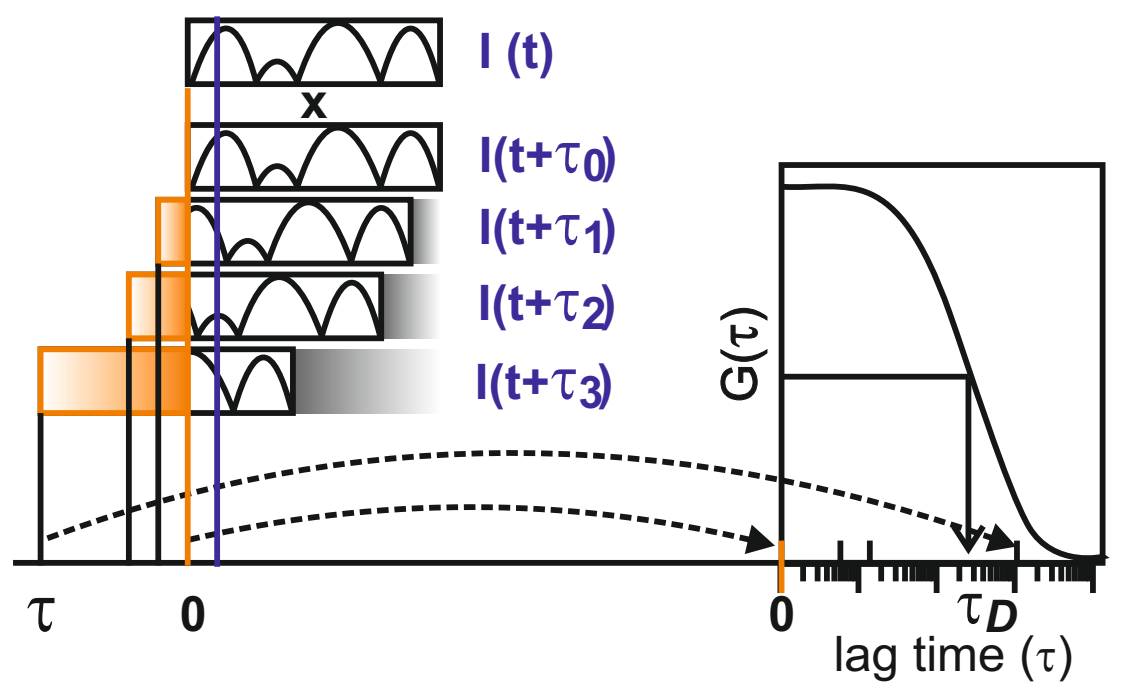

Figure 1.7: Fluorescence autocorrelation-spectroscopy. The autocorrelation function of a fluorescence signal $I(t)$ provides a quantitative measure of the similarity of this very signal with an instance of itself $I(t+\tau)$ shifted by a temporal offset $\tau$. A schematic fluorescence trace $I(t)$ and its time-shifted traces $I\left(t+\tau_{x}\right)(\mathrm{x}=0-3)$ are shown above (left). The autocorrelation $G(\tau)$ is calculated by multiplication of $I(t)$ with $I(t+\tau)$ over the entire trace (purple line), averaging the results and normalizing by the square of the average intensity. For $\tau=0, G(0)=\frac{1}{N}$ and at half of the decay $\tau$ $=\tau_{D}$. The temporal offset $\tau$, also referred to as the "correlation time", is the abscissa value and is usually represented in log scale (right). 
In this study, the liposomes and the SVs diffuse in a three-dimensional Brownian motion and the analytical expression of the autocorrelation function hence is [76]:

$$
G(\tau)=\frac{1}{\langle C\rangle \cdot \pi^{3 / 2} \cdot w_{0}^{2} \cdot z_{0}} \cdot\left(1+\frac{4 D \tau}{r_{0}^{2}}\right)^{-1} \cdot\left(1+\frac{4 D \tau}{z_{0}^{2}}\right)^{-1 / 2}
$$

In this equation, $\langle C\rangle$ is the average of the particle concentration in the effective focal volume $V_{\text {eff }}$, while $\pi^{3 / 2} \cdot w_{0}^{2} \cdot z_{0}$ is $V_{\text {eff }}$ as calculated by the integration of the emission distribution over the focal volume $V$. $D$ represents the molecular diffusion coefficient of the particle, and $w_{0}$ and $z_{0}$ the radius of the focal volume in lateral and axial directions, respectively. The focal volume is a property of the setup (laser beam and objective).

The time-independent part of this function can be expressed as $\frac{1}{N}$, where $N$ is the average particle number in $V_{\text {eff }}$ and can be calculated as $N=\langle C\rangle \cdot V_{e f f}$. The diffusion time $\tau_{D}$, for which a particle stays in the focal volume, is inversely proportional to the diffusion coefficient $D[\overline{30]}$ :

$$
\tau_{D}=\frac{w_{0}^{2}}{4 D} \quad \tau_{D, z}=\frac{z_{0}^{2}}{4 D}
$$

$\tau_{D}$ is the diffusion time in the lateral direction and $\tau_{D, z}$ in the axial direction. With these diffusion times, Equation 1.3 can be reformed as [50]:

$$
G(\tau)=\frac{1}{N} \cdot\left(1+\frac{\tau}{\tau_{D}}\right)^{-1} \cdot\left(1+\frac{\tau}{\tau_{D}} \cdot \frac{w_{0}^{2}}{z_{0}^{2}}\right)^{-1 / 2}
$$

The ratio of $\frac{w_{0}}{z_{0}}$ is a property of the experimental setup and was determined directly using immobilized gold beads $(\varnothing=20 \mathrm{~nm}$ ) on a micro-XYZ-stage for this study by Dr. W. H. Pohl [92]. The reflection of the laser beam by the gold beads can be observed only within the focal volume. The value of $\frac{w_{0}}{z_{0}}$ for the water immersion objective used in this study (UPlanSApo 60x/1.2w, Olympus) is 0.25 [92].

The time dependent part of the correlation function approaches unity at $\tau=0$, and thus the ordinate value gives the reciprocal of the particle number within the effective volume:

$$
G(0)=\frac{1}{N}
$$


At $\tau=\tau_{D}$, the time dependent part of the autocorrelation function is about 0.5 , neglecting the reciprocal square root, so that the diffusion time $\tau_{D}$ can be read off at the half-value of the decay. Figure 1.7 shows a schematic of the autocorrelation curve.

\subsubsection{Fluorescence cross-correlation spectroscopy (FCCS)}

In case of two-color experiments, two different dyes are used for labeling the sample particles, either in two different groups of sample components or at different positions of the same sample particles. The two dyes are excited simultaneously either with two (one-photon excitation, OPE) lasers or with a single (two-photon excitation, TPE) laser. Using fluorescence cross-correlation spectroscopy (FCCS), the number of interacting particles $N_{x}$ which accordingly emit both colors from spatial proximity after, for instance, fusion or tethering, can be determined. It should be noted at this point, that the interaction of the spatially close emitters might impact on the characteristics of the emission (fluorescence lifetime and intensity). However, for the immediate treatment of FCCS in this subsection, these effects shall not be considered. The FCCS function is analogous to the FCS function, so that a similarity comparison is performed between the separated fluorescence traces of two colors [50, 107]:

$$
G_{R G}(\tau)=\frac{\left\langle\delta I_{R}(t) \cdot \delta I_{G}(t+\tau)\right\rangle}{\left\langle I_{R}(t)\right\rangle \cdot\left\langle I_{G}(t)\right\rangle}
$$

$G_{R G}(\tau)$ is the amplitude of the cross-correlation. $I_{R}(t)$ and $I_{G}(t)$ are the intensity functions of the separate red and green channels (Figure 1.8).

Equation 1.5 is fitted to the cross-correlation data and, from the resulting $G_{R G}(0)$, the multicolor particle number $N_{x}$ can be calculated as [99]:

$$
G_{R G}(0)=\frac{N_{x}}{\left(N_{R}+N_{x}\right) \cdot\left(N_{G}+N_{x}\right)}
$$

$N_{R}$ and $N_{G}$ are the numbers of the free red and green emitters, respectively.

The sum of $N_{i}(i=R, G)$ and $N_{x}$ can be calculated from the autocorrelation of the 
respective channel:

$$
\begin{aligned}
& N_{R}+N_{x}=N_{R}(0)=\frac{1}{G_{R}(0)} \\
& N_{G}+N_{x}=N_{G}(0)=\frac{1}{G_{G}(0)}
\end{aligned}
$$

$N_{x}$ can subsequently be obtained as:

$$
N_{x}=G_{R G}(0) \cdot N_{R}(0) \cdot N_{G}(0)
$$

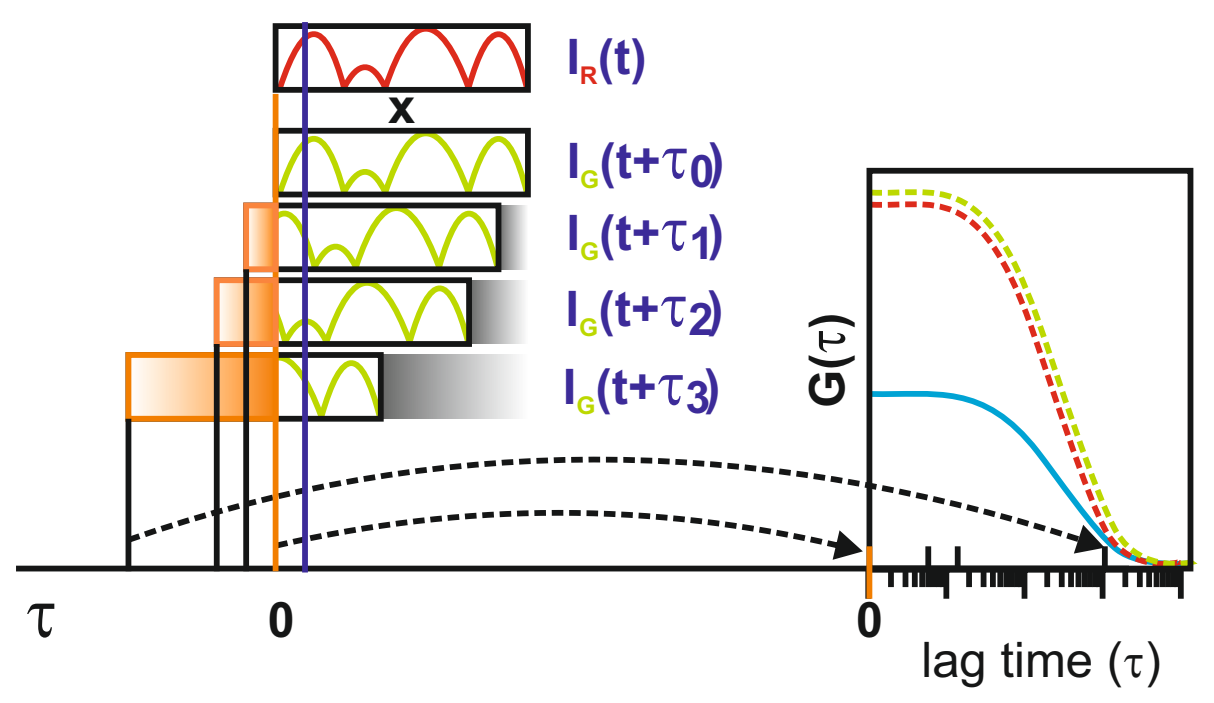

Figure 1.8: Fluorescence cross-correlation-spectroscopy. The FCCS concept is analogous to FCS but with two separate fluorescence channels (left). From the results of FCCS (blue solid line) and of FCS for each color (red and green dash lines), the number of the particles with both red and green dyes $N_{x}$ can be calculated (right).

\subsubsection{Two-photon excitation (TPE)}

FCS measurements require a low sample concentration $(\mathrm{nM})$ and a small detection volume, so that a fluctuation of the individual dye's emission can be observed clearly. 
The latter can be achieved using an objective with high numerical aperture (NA, $\mathrm{NA}=1.2$ in this study) in a confocal microscope [100], minimizing the lateral extent of the excitation beam to several $10^{2} \mathrm{~nm}$. Furthermore, a pinhole is needed to limit the axial dimension of the focal volume. Alternatively, two-photon excitation (TPE), due to its excitation property, can be used for FCS to reduce the excitation volume and thus the effective volume of the focus $[10,23,27,55]$.

During two-photon excitation, the fluorescence dye absorbs, in one excitation process, two photons of theoretically double the wavelength used for one-photon excitation within a sub-femtosecond time range (Figure 1.93) [44]. To achieve this, a pulsed laser is required for drastically increasing the photon density in the focus area. The excitation probability is proportional to the square of the laser's intensity, so that only the middle part of the focal volume can be excited. The distribution of the emission light depends on the laser beam and the objective, and can be approximated as a 3DGaussian. Thus the effective excitation volume is decreased by a factor of $\sqrt{2}$ in each direction for TPE:

$$
\begin{array}{ll}
\text { OPE: } & V_{\text {eff }}=\pi^{3 / 2} \cdot w_{0}^{2} \cdot z_{0} \quad \text { (Page 13, Subsection 1.5.1) } \\
\text { TPE: } & V_{\text {eff }}=\left(\frac{\pi}{2}\right)^{3 / 2} \cdot w_{0}^{2} \cdot z_{0}
\end{array}
$$

and the diffusion time is accordingly shorter by a factor of two in each direction (Figure $1.9 \mathrm{p})$ :

$$
\begin{aligned}
& \text { OPE: } \quad \tau_{D}=\frac{w_{0}^{2}}{4 D} \quad \tau_{D, z}=\frac{z_{0}^{2}}{4 D} \quad(\text { Page } 13 \text {, Equation 1.4 } \\
& \mathrm{TPE}: \quad \tau_{D}=\frac{w_{0}^{2}}{8 D} \quad \tau_{D, z}=\frac{z_{0}^{2}}{8 D}
\end{aligned}
$$

The typical $V_{e f f}^{O P E}$ for OPE is about $1 \mathrm{fl}$.

For our two-photon confocal microscope, the lateral radius $w_{0}$ was determined using the fluorescence dye Rhodamine Green (RG) with a known diffusion coefficient $D_{R G}=2.8 \times 10^{-6} \mathrm{~cm}^{2} \mathrm{~s}^{-1}[24,100]$ and calculated using equation 1.15 . The $V_{e f f}^{T P E}$ was calculated with equation 1.13 and the value is about $0.3 \mathrm{fl}$, which perfectly confirmed the expected decrease in detection volume by a factor of $\sqrt{8}$ ( $\sqrt{2}$ in each direction) compared to OPE. 

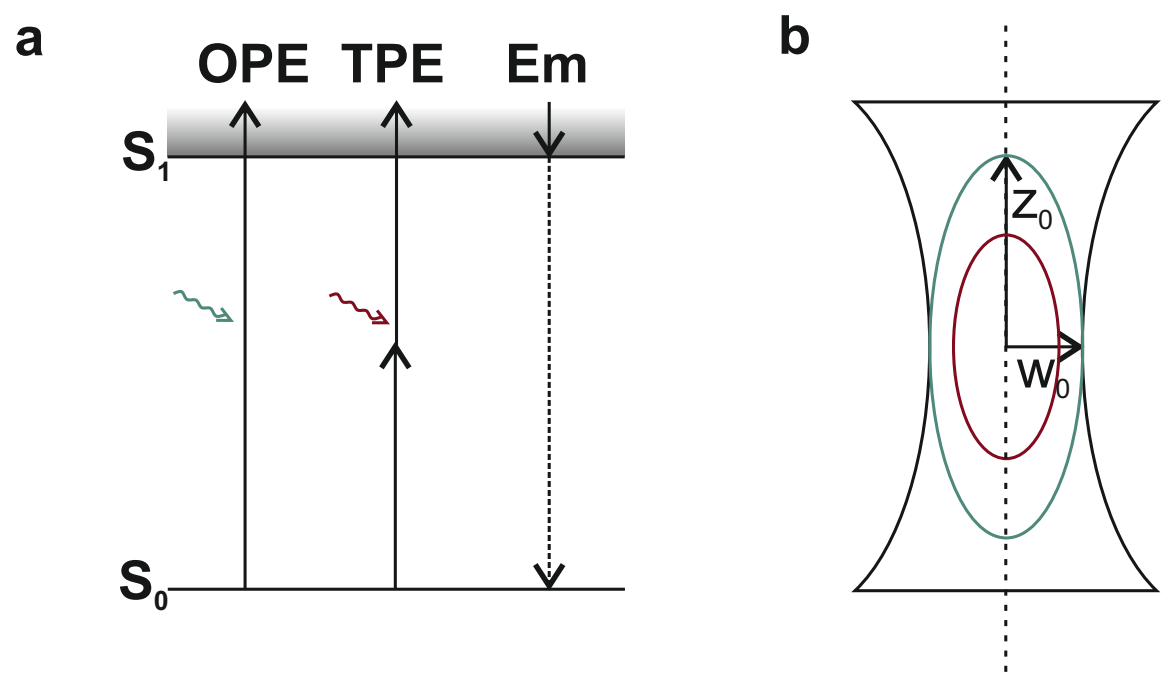

Figure 1.9: OPE compared with TPE in terms of the excitation module and the effective volume. a. Jablonski-diagram for OPE and TPE. b. Schematic of the effective excitation volume by OPE (green) and TPE (red).

Two-photon excitation allows simultaneous excitation of two fluorescence dyes with one laser beam and protects the biological sample against denaturation throughout most of the path of the laser beam due to its low photon energy and its small excitation volume.

\subsubsection{Förster resonance energy transfer (FRET)}

Förster resonance energy transfer (FRET) is a non-radiative energy transfer between a donor fluorophore and an acceptor fluorophore. The donor is excited to its electronically excited state, transfers the energy to the acceptor nearby and thus excites the acceptor [41]. In this process, the donor fluorescence is quenched and only the acceptor is able to fluoresce (Figure 1.10). For FRET to occur, the spectra of the donor emission and the acceptor absorption need to overlap, the distance between the donor and the acceptor has to be relatively short (10-100 $\AA$ ), and relative dipole-dipole orientations have to be favorable for coupling [122].

In general, the experimentally observable rate constant $k_{\exp }$ of the decay of the 


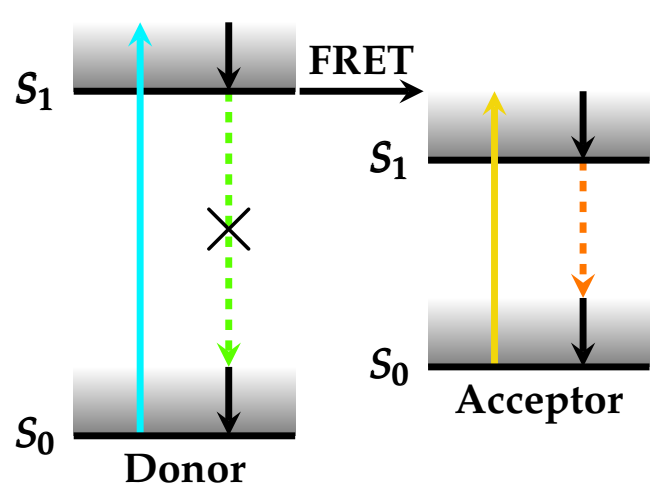

Figure 1.10: Jablonski-diagram of FRET donor and acceptor. As the excited state's energy is transferred from the donor to the acceptor, donor emission is quenched and acceptor fluorescence enhanced.

donor's (D) and acceptor's (A) respective $S_{1}$ state populations is a sum of the fluorescence ( $\mathrm{Fl})$ rate constant and other rate constants, for instance internal conversion (IC), intersystem crossing (ISC):

$$
\begin{aligned}
& k_{\text {exp }}^{D}=k_{F l}^{D}+k_{I C}^{D}+k_{I S C}^{D} \\
& k_{\text {exp }}^{A}=k_{F l}^{A}+k_{I C}^{A}+k_{I S C}^{A}
\end{aligned}
$$

If FRET occurs, the depletion of the donor's electronically excited state is accelerated:

$$
k_{\text {exp }}^{D^{\prime}}=k_{F l}^{D}+k_{I C}^{D}+k_{I S C}^{D}+k_{F R E T}
$$

The fluorescence quantum efficiency can be calculated as $k_{F l} / k_{\exp }$ and the observed fluorescence lifetime $\tau_{\exp }$ is the inversion of the emission rate constant $k_{\text {exp }}$. Thus, the donor emission count rate and lifetime $\tau_{D}$ decrease under FRET.

The FRET distance is the donor-acceptor-distance at which the FRET quantum efficiency $k_{F R E T} / k_{\text {exp }}$ is $50 \%$ [11]. FRET is, of course, most pronounced below this distance. In the Syt1 tethering experiment, the liposomes were labeled with either 1\% TR-DHPE or 1.5\% OG-DHPE in such a way that upon fusion the distance between TR and OG is about $7 \mathrm{~nm}$. According to a previous study using TR-OG FRET pair [23,55], FRET can be observed upon fusion. Figure 1.11 shows the excitation and emission spectra of TR 
and OG.

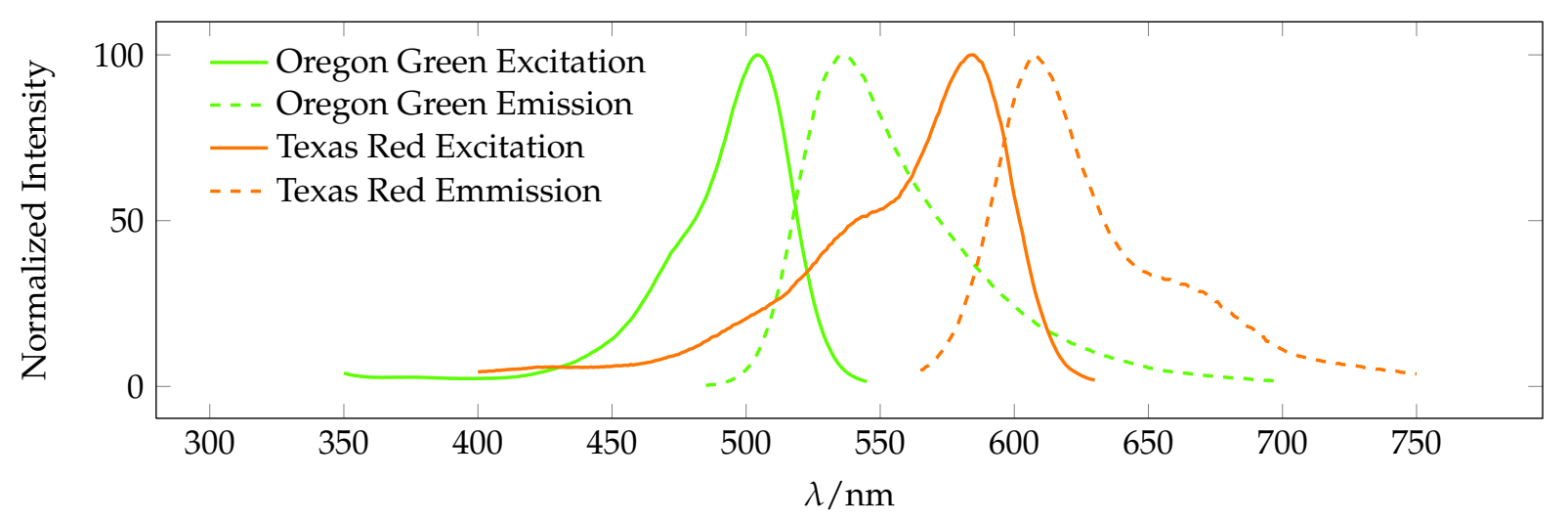

Figure 1.11: Excitation and emission spectra of TR-DHPE and OG-DHPE.

\subsection{Outline of this Study}

This study focuses on the functional mechanism of Syt1 with the particular emphasis on the cis- and trans-membrane interaction of Syt1. To achieve this, molecular requirements for the calcium dependent and independent binding properties are investigated using reconstituted liposomes with a sensitive tethering assay based on two photon-fluorescence cross correlation spectroscopy (TP-FCCS). Tethering is measured between the liposomes bearing Syt1 and the liposomes containing acidic phospholipids, in this case phosphatidylserine (PS) and phosphatidylinositol-4,5-bisphosphate (PiP2), under the influence of calcium. The host liposomes of Syt1 contain either no acidic phospholipids or $20 \%$ PS to obtain either trans- or cis-binding. Furthermore, interaction between membrane anchored Syt1 and membrane anchored SNARE proteins (Syntaxin 1A (Sx1A), a 2:1 complex consisting of two Sx1A and one SNAP-25, and a ternary complex consisting of Sx1A, SNAP-25 and Synaptobrevin 2 (Sb2) lacking transmembrane domain) is tested. To investigate the binding function of different binding sites, mutants at $\mathrm{C} 2 \mathrm{~A}$ and $\mathrm{C} 2 \mathrm{~B}$ domains of the full-length Syt 1 are used instead of the wild-type Syt1. In addition, cross-linking mediated by soluble Syt1 (aa 1-97) is measured to complete the study by comparison with the previous publications. 
Associated with the Syt1 project, two preliminary measurements are performed in this study, aimed at characterizing the mouse synaptic vesicle (SVs) by average protein mass, and monitoring the chromaffin granules (CGs) docking with the large liposomes mediated by partial SNARE assembly arrested by $\alpha$-SNAP. Purified mouse SVs are labeled with FM 1-43 fluorescence dye and measured with fluorescence correlation spectroscopy (FCS) to determine the vesicle number. Combining the protein concentration of the same sample, an average vesicular mass of proteins can be calculated. This experiment introduces a nice comparison with the previous study of rat SVs and establishes a vesicular base for the further characterization of the composition of proteins as well as lipids in a single vesicle manner.

Using FCCS tethering assay, a stable docked state between CGs and large liposomes can be monitored for the first time. $\alpha$-SNAP is reported to be able to inhibit CG fusion by the lipid mixing assay. Here, docking can be observed under the same conditions. This observation suggests a partial assembly model of the SNARE complex mediated by $\alpha$-SNAP, which inhibits CG fusion, but not CG docking. 


\section{Chapter 2}

\section{Materials and Methods}

\subsection{Materials}

\subsubsection{Chemicals}

All the standard chemicals used in this study were purchased from the following companies: Sigma (Deisenhofen, Germany), Sigma-Aldrich (St. Louis, USA), AppliChem (Darmstadt, Germany), Merck (Darmstadt, Germany), Roth (Karlsruhe, Germany), Biorad (Richmond, USA), Serva (Heidelberg, Germany), Boehringer (Ingelheim, Germany ), Fluka (Switzerland) and Anatrace (USA). The column material Sephadex G50 was purchased from GE Healthcare (Freiburg, Germany) and the molecular weight protein standards (SM0671) was purchased from MBI Fermentas (St. LeonRot, Germany).

\subsubsection{Phospholipids}

All the phospholipids used in the tethering experiments were purchased from Avanti Polar Lipids Inc. (Alabama, USA), except Texas Red phosphatidylethanolamine and Oregon Green phosphatidylethanolamine, which were purchased from Invitrogen 
Molecular Probes (see Table 2.1).

\begin{tabular}{l|ll}
\hline \hline Abbreviation & Phospholipid & Company \\
\hline PC & L- $\alpha$-Phosphatidylcholine & Avanti Polar Lipids \\
PE & L- $\alpha$-Phosphatidylethanolamine & Avanti Polar Lipids \\
PS & L- $\alpha$-Phosphatidylserine & Avanti Polar Lipids \\
PI & L- $\alpha$-Phosphatidylinositol & Avanti Polar Lipids \\
PiP2 & Phosphatidylinositol-4,5- & Avanti Polar Lipids \\
& bisphosphate & \\
TRPE & Texas Red-PE & Invitrogen Molecular Probes \\
OGPE & Oregon Green-PE & Invitrogen Molecular Probes \\
NBD-DOPE & 1,2-dioleoyl-sn-glycero-3- & Avanti Polar Lipids \\
& phosphoethanolamine- $N-(7-$ & \\
& nitrobenz-2-oxa-1,3-diazol-4-yl) & \\
Rhodamine-DOPE & 1,2-dioleoyl-sn-glycero-3- & Avanti Polar Lipids \\
& phosphoethanolamine- $N-$ & \\
& lissamine rhodamine B sulfonyl & \\
& ammonium salt & \\
\hline \hline
\end{tabular}

Table 2.1: Phospholipids used for reconstitution of liposomes.

\subsubsection{Proteins}

All the protein constructs used in this study were from Rattus norvegicus. The proteins were cloned in the pET28a (Novagen) vector and expressed in Escherichia coli strain BL21 (DE3) except the $\Delta \mathrm{N}$ complex, in which syntaxin 1A (Sx1A) (183-288) and synaptobrevin 2 (49-96) were cloned in pET Duet-1 (Novagen), and SNAP 25A was cloned in pET28a.

Expression constructs of the full-length synaptotagmin-1 (Syt1) (1-421) and of the soluble domain of Syt1 (97-421), have been described elsewhere [111]. Also the 
following calcium mutants of the full-length Syt1 have been described earlier [111]: $\mathrm{a}^{*} \mathrm{~B}(\mathrm{D} 178,230,232 \mathrm{~A}), \mathrm{Ab}^{*}(\mathrm{D} 309,363,365 \mathrm{~A}), \mathrm{a}^{*} \mathrm{~b}^{*}(\mathrm{D} 178,230,232,309,363,365 \mathrm{~A})$, and KAKA mutant (K326, 327A). The constructs for the neuronal SNAREs were the SNARE motif of syntaxin 1A with its transmembrane domain (183-288), a cysteine-free variant of SNAP-25A (1-206), and synaptobrevin 2 without its transmembrane domain (1-96). The Syt1 (97-421) single cysteine variant (S342C) was obtained after first removing the single native cysteine (C278S) and then introducing a point mutation at position 342.

All proteins were expressed in Escherichia coli strain BL21 (DE3) (Novagen) and purified using $\mathrm{Ni}^{2+}$-nitrilotriacetic acid beads (Ni-NTA, GE Healthcare) followed by ion exchange chromatography (IEXC) on the Aekta system (GE Healthcare) as described in the literature [91, 111], with a few modifications. The Syt1 single cysteine variant (97-421, S342C) was further labeled with Alexa Fluor 488 C5 maleimide. This was done by first dialyzing the proteins against the AF-labeling buffer (page 28, Table 2.3. . The dialyzed protein solution was then incubated with the fluorophore for $2 \mathrm{~h}$ at RT and separated from the free dye using a Sephadex G50 superfine column. The labeling efficiency was about $40 \%[111,140,141]$. Sx1A (183-288) and synaptobrevin 2 (1-96) were purified by IEXC in the presence of $15 \mathrm{mM} \mathrm{CHAPS}$. The binary complex (2:1) containing Sx1A (183-288) and SNAP-25A (1-206) was assembled from purified monomers and subsequently purified by IEXC in the presence of $1 \%$ CHAPS. The $\Delta \mathrm{N}$ complex was co-expressed with pET Duet-1 and pET28a vectors and purified by IEXC with $50 \mathrm{mM}$ n-octyl- $\beta$-D-glucoside [91]. The ternary SNARE complex consisting of Sx1A (183-288), SNAP-25A and synaptobrevin 2 (1-96) was generated by incubating of the binary complex and synaptobrevin $2(1-96)$ in a ratio of 1:2 over night at $4^{\circ} \mathrm{C}$. The excess synaptobrevin 2 was removed with Sephadex G50 superfine column during liposome reconstitution.

All the SNARE proteins including different SNARE complexes, and $\alpha$-SNAP and its mutants were provided by Dr. Alexander Stein and Dr. Yongsoo Park (neurobiology department) [33, 35, 91, 105, 111, 128, 132]. Full-length Syt1 (Syt1/1-421/PET28a/NdeIXhoI/His) was cloned by the neurobiology department and expressed in this study. 
The expression and purification were described in page 30, subsection 2.2.1. The Syt1 purification protocol was modified from the publications and the dissertation of Dr. Alexander Stein [95, 111]. The mutants of the full-length Syt1 and the soluble Syt1 were provided by Dr. Geert van den Bogaart [111, 140, 141]. The sequences of all the proteins are given in Table 2.2 .

\begin{tabular}{|c|c|c|c|c|}
\hline Abbreviation & Protein & Sequence & & \\
\hline $\mathrm{Sb}^{1-96}$ & Synaptobrevin 2 1-96 & $1-96$ & 35 & \\
\hline SNAP25A & SNAP 25A & $1-206, \mathrm{C} 84,85,90,92 \mathrm{~S}$ & [33] & \\
\hline Sx1A & Syntaxin $1 \mathrm{~A}$ & $183-288$ & [105] & \\
\hline$\Delta \mathrm{N}$ complex & $\begin{array}{l}\text { Sx1A } \\
\text { SNAP25A } \\
\text { Sb2 49-96 }\end{array}$ & $\begin{array}{l}183-288 \\
1-206, C 84,85,90,92 S \\
49-96\end{array}$ & [91] & \\
\hline 2:1 complex & $\begin{array}{l}\text { 2x Sx1A } \\
1 \times \text { SNAP25A }\end{array}$ & $\begin{array}{l}183-288 \\
1-206, \mathrm{C} 84,85,90,92 \mathrm{~S}\end{array}$ & {$[111$} & 128 \\
\hline Syt1 & Synaptotagmin-1 & $1-421$ & \multicolumn{2}{|c|}{ this study } \\
\hline$a^{*} B$ & Synaptotagmin $-1 \mathrm{a}^{*} \mathrm{~B}$ & $1-421, \mathrm{D} 178,230,232 \mathrm{~A}$ & & \\
\hline $\mathrm{Ab}^{*}$ & Synaptotagmin-1 $\mathrm{Ab}^{*}$ & $1-421, \mathrm{D} 309,363,365 \mathrm{~A}$ & & \\
\hline$a^{*} b^{*}$ & Synaptotagmin-1 $\mathrm{a}^{*} \mathrm{~b}^{*}$ & $\begin{array}{l}1-421, \mathrm{D} 178,230,232 \\
309,363,365 \mathrm{~A}\end{array}$ & {$[111$} & 140,141 \\
\hline KAKA & Synaptotagmin-1 KAKA & $1-421, \mathrm{~K} 326,327 \mathrm{~A}$ & & \\
\hline $\mathrm{C} 2 \mathrm{AB}$ & Soluble synaptotagmin-1 & $97-421$ & & \\
\hline $\mathrm{AF}-\mathrm{C} 2 \mathrm{AB}$ & Soluble synaptotagmin-1 & $\begin{array}{l}\text { 97-421, S342C-Alexa } \\
\text { Fluor } 488\end{array}$ & & \\
\hline$\alpha$-SNAP & $\alpha$-SNAP & $1-295$ & & \\
\hline$\alpha-\mathrm{SNAP}^{33-295}$ & $\alpha$-SNAP deletion & 33-295 & {$[132$} & \\
\hline$\alpha-\mathrm{SNAP}^{F 27,28 S}$ & $\alpha$-SNAP mutation & $1-295$, F27, $28 S$ & & \\
\hline
\end{tabular}

Table 2.2: Proteins used in this study. 


\subsubsection{Buffers}

The buffers used throughout this study are listed in Table 2.3 .

Table 2.3: Buffers used in this study.

\begin{tabular}{l|rl}
\hline \hline Buffer & Concentration & Components \\
\hline Chloroform/MeOH & $2 / 3(\mathrm{v} / \mathrm{v})$ & Chloroform \\
& $1 / 3(\mathrm{v} / \mathrm{v})$ & MeOH \\
\hline DTT stock solution & $1 \mathrm{mM}$ & DTT in ddH ${ }_{2} \mathrm{O}$ (Milli-Q) \\
\hline HP150 & $20 \mathrm{mM}$ & HEPES \\
& $150 \mathrm{mM}$ & KCl \\
& $2 \mathrm{mM}$ & DTT \\
\hline HPCholate5 & $20 \mathrm{mM}$ & HEPES \\
& $150 \mathrm{mM}$ & KCl \\
& $2 \mathrm{mM}$ & DTT \\
& $5 \%(\mathrm{w} / \mathrm{v})$ & Sodium Cholate \\
& $20 \mathrm{mM}$ & HEPES \\
& $150 \mathrm{mM}$ & KCl \\
& $2 \mathrm{mM}$ & DTT \\
& $1.5 \%(\mathrm{w} / \mathrm{v})$ & Sodium Cholate \\
\hline HPCholate1.5 & & \\
& &
\end{tabular}

Tethering experiments

\begin{tabular}{l|rl}
\hline EGTA buffer & $20 \mathrm{mM}$ & HEPES \\
$150 \mathrm{mM}$ & $\mathrm{KCl}$ \\
$2 \mathrm{mM}$ & DTT \\
$1 \mathrm{mM}$ & EGTA \\
\hline
\end{tabular}

continued on next page 


\begin{tabular}{l|rl}
\hline Buffer & Concentration & Components \\
\hline Calcium buffer & $20 \mathrm{mM}$ & HEPES \\
$150 \mathrm{mM}$ & $\mathrm{KCl}$ \\
$2 \mathrm{mM}$ & DTT \\
$1 \mathrm{mM}$ & EGTA \\
& $1.1 \mathrm{mM}$ & $\mathrm{CaCl}_{2}$ \\
\hline CG buffer & $20 \mathrm{mM}$ & HEPES, $\mathrm{pH} 7.4$ \\
& $120 \mathrm{mM}$ & potassium glutamate \\
& $20 \mathrm{mM}$ & potassium acetate \\
& $5 \mathrm{mM}$ & MgCl $_{2}$ \\
\hline
\end{tabular}

Purification of synaptotagmin-1 (1-421)

\begin{tabular}{l|rl}
\hline PMSF stock solution & $200 \mathrm{mM}$ & PMSF in EtOH \\
\hline Extraction buffer & $20 \mathrm{mM}$ & Tris, pH 7.4 \\
& $500 \mathrm{mM}$ & $\mathrm{NaCl}$ \\
& $20 \mathrm{mM}$ & Imidazole \\
\hline Wash buffer & $20 \mathrm{mM}$ & Tris, pH 7.4 \\
& $500 \mathrm{mM}$ & $\mathrm{NaCl}$ \\
& $20 \mathrm{mM}$ & Imidazole \\
& $1 \%(\mathrm{w} / \mathrm{v})$ & CHAPS \\
\hline Elution buffer & $20 \mathrm{mM}$ & Tris, pH 7.4 \\
& $500 \mathrm{mM}$ & NaCl \\
& $400 \mathrm{mM}$ & Imidazole \\
& $1 \%(\mathrm{w} / \mathrm{v})$ & CHAPS \\
\hline Thrombin stock solution & $5 \mathrm{mg} / \mathrm{ml}$ & Trombin in 50\% (v/v) Glycerol \\
\hline
\end{tabular}

continued on next page 


\begin{tabular}{l|rl}
\hline Buffer & Concentration & Components \\
\hline Desalting buffer & $20 \mathrm{mM}$ & Tris, $\mathrm{pH} 7.4$ \\
$300 \mathrm{mM}$ & $\mathrm{NaCl}$ \\
& $1 \%(\mathrm{w} / \mathrm{v})$ & CHAPS \\
$1 \mathrm{mM}$ & EDTA \\
& $1 \mathrm{mM}$ & DTT \\
\hline
\end{tabular}

Ion Exchange Chromatography (IEXC)

\begin{tabular}{|c|c|c|}
\hline \multirow[t]{3}{*}{ Buffer A } & $20 \mathrm{mM}$ & Tris, pH 7.4 \\
\hline & $1 \mathrm{mM}$ & DTT \\
\hline & $1 \%(\mathrm{w} / \mathrm{v})$ & CHAPS \\
\hline \multirow[t]{4}{*}{ Buffer B } & $20 \mathrm{mM}$ & Tris, pH 7.4 \\
\hline & $1 \mathrm{mM}$ & DTT \\
\hline & $1 \%(w / v)$ & CHAPS \\
\hline & $1 \mathrm{M}$ & $\mathrm{NaCl}$ \\
\hline \multicolumn{3}{|c|}{ Schägger-gel for SDS-PAGE [108] } \\
\hline \multirow[t]{5}{*}{ Sample buffer } & $50 \mathrm{mM}$ & Tris, pH 6.8 \\
\hline & $4 \%(\mathrm{w} / \mathrm{v})$ & SDS \\
\hline & $0.01 \%(\mathrm{w} / \mathrm{v})$ & Serva Blue G \\
\hline & $12 \%(\mathrm{v} / \mathrm{v})$ & Glycerol \\
\hline & $2 \%(v / v)$ & $\beta$-Mercaptoethanol \\
\hline \multirow[t]{2}{*}{ Gel buffer } & $3 \mathrm{M}$ & Tris, pH 8.45 \\
\hline & $0.3 \%(\mathrm{w} / \mathrm{v})$ & SDS \\
\hline Anode buffer & $2 \mathrm{M}$ & Tris, pH 8.9 \\
\hline \multirow[t]{3}{*}{ Cathode buffer } & $1 \mathrm{M}$ & Tris \\
\hline & $1 \mathrm{M}$ & Tricine \\
\hline & $1 \%(\mathrm{w} / \mathrm{v})$ & SDS \\
\hline
\end{tabular}




\begin{tabular}{l|rl}
\hline Buffer & Concentration & Components \\
\hline Coomassie staining solution & $0.2 \%(\mathrm{w} / \mathrm{v})$ & Coomassie Brilliant Blue $\mathrm{R}$ \\
& $25 \%(\mathrm{v} / \mathrm{v})$ & $\mathrm{EtOH}$ \\
$10 \%(\mathrm{v} / \mathrm{v})$ & Acetic acid \\
& $65 \%(\mathrm{v} / \mathrm{v})$ & $\mathrm{ddH}_{2} \mathrm{O}$ \\
\hline Coomassie destaining solution & $20 \%(\mathrm{v} / \mathrm{v})$ & EtOH \\
& $5 \%(\mathrm{v} / \mathrm{v})$ & Acetic acid \\
& $1 \%(\mathrm{v} / \mathrm{v})$ & Glycerol \\
\hline
\end{tabular}

\section{SV purification}

\begin{tabular}{|c|c|c|}
\hline \multirow[t]{3}{*}{ HB-100 } & $25 \mathrm{mM}$ & HEPES, pH 7.4 \\
\hline & $100 \mathrm{mM}$ & $\mathrm{KCl}$ \\
\hline & $1 \mathrm{mM}$ & DTT \\
\hline \multicolumn{3}{|c|}{ Lowry-Peterson protein determination $[90]$} \\
\hline \multirow[t]{4}{*}{ Lowry solution I } & $189 \mathrm{mM}$ & $\mathrm{Na}_{2} \mathrm{CO}_{3}$ \\
\hline & $68 \mathrm{mM}$ & $\mathrm{NaOH}$ \\
\hline & $8 \mathrm{mM}$ & $\mathrm{Na}_{2}$-Tartrate $\cdot 2 \mathrm{H}_{2} \mathrm{O}$ \\
\hline & $1 \%(\mathrm{w} / \mathrm{v})$ & SDS \\
\hline hline Lowry solution II & $250 \mathrm{mM}$ & $\mathrm{CuSO}_{4} \cdot 5 \mathrm{H}_{2} \mathrm{O}$ \\
\hline \multirow[t]{2}{*}{ hline Lowry solution III } & $100 \mathrm{ml}$ & Lowry solution I \\
\hline & $1 \mathrm{ml}$ & Lowry solution II \\
\hline \multirow[t]{2}{*}{ Lowry solution IV } & $2 \mathrm{~N}$ & Folin-Ciocalteu's phenol reagent \\
\hline & $1: 1$ & diluted with dd $\mathrm{H}_{2} \mathrm{O}$ \\
\hline \multicolumn{3}{|c|}{ Labeling buffers } \\
\hline FM 1-43 stock solution & $1 \mathrm{mM}$ & $\mathrm{FM} 1-43$ in $\mathrm{ddH}_{2} \mathrm{O}$ \\
\hline \multirow[t]{3}{*}{ AF-labeling buffer } & $50 \mathrm{mM}$ & HEPES, pH 7.4 \\
\hline & $500 \mathrm{mM}$ & $\mathrm{NaCl}$ \\
\hline & $100 \mu \mathrm{M}$ & Tris(2-carboxyethyl)phosphine \\
\hline
\end{tabular}




\subsubsection{Instruments, filters, columns and others}

The most important instruments and the filters as well as the columns for protein purification and for the tethering experiments are listed in Table 2.4 .

Table 2.4: Instruments, filters, columns and miscellanea.

\begin{tabular}{|c|c|}
\hline Item & Supplier \\
\hline \multicolumn{2}{|l|}{ Instruments } \\
\hline Electrophoresis chamber Mini-Protean II & Biorad (Richmond, USA) \\
\hline Power Pac 300 & Biorad (Richmond, USA) \\
\hline Nanodrop-Spectrometer 1000 & Thermo Scientific (Germany) \\
\hline Novaspec II photometer & Parmacia Biotech (Germany) \\
\hline Cary 5E, UV-Vis-NIR & Varian (Germany) \\
\hline Aekta system & GE Healthcare (Germany) \\
\hline J6-MI Centrifuge & Beckman Coulter (Germany) \\
\hline Sorvall RC-5B Refrigerated Superspeed Centrifuge & Thermo Scientific (Germany) \\
\hline Sorvall SS34 & Thermo Scientific (Germany) \\
\hline Sorvall F14-6x250y & Thermo Scientific (Germany) \\
\hline SW41T1 rotor & Beckman Coulter (Germany) \\
\hline
\end{tabular}

\section{Components for FCS measurements}

Chameleon Ti:Sa laser system

Solid State Thermoelectric Thermal Control Unit $\mathrm{T} 225 \mathrm{P}$

IX71 inverted microscope

UPlanSApo 60x/1.2w water immersion objective

Avalanche photodiode (APD, SPCM-AQR-13)

PRT 400, 4-channel router

TimeHarp200, TCSPC card
Coherent (California, USA)

Coherent (California, USA)

Olympus (Germany)

Olympus (Germany)

Perkin-Elmer (Canada)

PicoQuant GmbH (Germany)

PicoQuant GmbH (Germany) 


\begin{tabular}{|c|c|}
\hline Item & Supplier \\
\hline \multicolumn{2}{|l|}{ Filters } \\
\hline 715 DSCPXR dichroic mirror & AHF (Germany) \\
\hline 590 DCXR dichroic mirror & AHF (Germany) \\
\hline E700SP2 short pass filter & AHF (Germany) \\
\hline Ultra-broadband dielectric mirror, $650-1130 \mathrm{~nm}$ & Newport (USA) \\
\hline HQ 645/75 bandpass filter & AHF (Germany) \\
\hline HQ 535/50 bandpass filter & AHF (Germany) \\
\hline \multicolumn{2}{|l|}{ Columns } \\
\hline Sephacryl S-1000 Superfine HR & GE Healthcare (Germany) \\
\hline Econo-column, $0.5 \mathrm{~cm} \times 10 \mathrm{~cm}$ & Biorad, (Richmond, USA) \\
\hline Econo-column, $2.5 \mathrm{~cm} \times 10 \mathrm{~cm}$ & Biorad, (Richmond, USA) \\
\hline \multicolumn{2}{|l|}{ Miscellanea } \\
\hline Coverslip $18 \times 18 \mathrm{~mm}$ & Menzel-Gläser (Germany) \\
\hline $\mathrm{Ni}^{2+}$-nitrilotriacetic acid beads (Ni-NTA) & GE Healthcare (Germany) \\
\hline
\end{tabular}

\subsubsection{Software}

The software products used for data analysis were MATLAB 2009b (The MathWorks, Inc.), Origin 8.6G (MicroCal Inc.), Microsoft Office Suite 2010 (Microsoft Corp.) and SigmaPlot (Systat Software Inc.).

\subsection{Methods}

\subsubsection{Expression and purification of synaptotagmin-1}

Synaptotagmin-1 (Syt1) was cloned in the pET28a vector (Novagen) and transformed via heat shock in the expression cell Escherichia coli strain BL21 (DE3, Novagen, see page 22, subsection 2.1.3). Transformation was performed with the standard pro- 
tocol of the neurobiology department. To check the expression condition, four $50 \mathrm{ml}$ cultures were prepared in the TB-medium containing $30 \mu \mathrm{g} / \mathrm{ml}$ kanamycin. The cultures grew under shaking at $37^{\circ} \mathrm{C}$ until $\mathrm{OD}=0.8-1.0$. The expression was started with IPTG inducing at a concentration of $0.5 \mathrm{mM}$ and performed either at $37^{\circ} \mathrm{C}$ or at $22^{\circ} \mathrm{C}$ for $3 \mathrm{~h}$ as well as over night $(\mathrm{ON})$.

The expression was checked with SDS-PAGE (Figure 2.1). The best condition for the Syt 1 expression was at $22^{\circ} \mathrm{C}$ for at least $3 \mathrm{~h}$ after IPTG inducing. After expression, the culture was centrifuged (4000 RPM, $15 \mathrm{~min}, 4^{\circ} \mathrm{C}$ ) and the pellet was resuspended with a little amount of extraction buffer (all buffers used in this subsection see page 26 . Table 2.3). The resuspended cell was kept at $-20^{\circ} \mathrm{C}$.

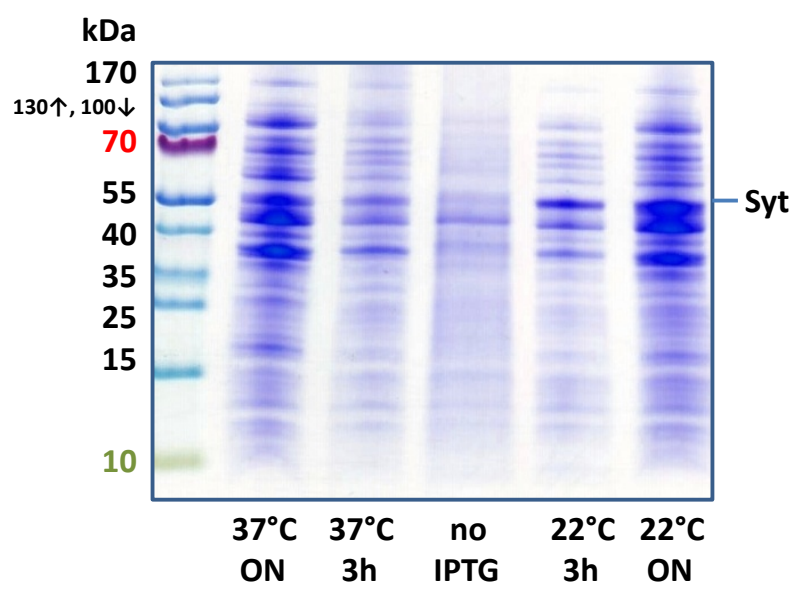

Figure 2.1: Expression test of Syt1 under different conditions. Each $50 \mathrm{ml}$ TB-KANA-culture $(30 \mu \mathrm{g} / \mathrm{ml})$ was incubated at $37^{\circ} \mathrm{C}$ until $\mathrm{OD}=0.8-1.0$. The expression was started with $0.5 \mathrm{mM}$ IPTG and performed under different conditions (shown on the gel picture). The sample in the middle ("no IPTG") was taken before adding IPTG.

Syt1 was purified with immobilized metal ion affinity chromatography (IMAC) followed by Ion Exchange Chromatography (IEXC). The resuspended cell pellet was thawed and filled with the same amount of extraction buffer with $10 \%$ sodium cholate. lysozyme (f.c. $1 \mathrm{mg} / \mathrm{ml}$ ), PMSF $1 \mathrm{mM}$ ), $\mathrm{MgCl}_{2}$ (f.c. $1 \mathrm{mM}$ ) and a few crumbs of DNAse I were added to the cell solution and the mixture was incubated under stirring for $30 \mathrm{~min}$ at RT. The lysate was furthermore homogenized with $4 \times 40$ strokes using ultra- 
sound (large Tip, 50\% duty cycle, microtip limit) and incubated for $15 \mathrm{~min}$ at RT. The solution was centrifuged at $15000 \mathrm{G}$ for $30 \mathrm{~min}$ (SLA-1500).

The supernatant was carefully collected and mixed with $9 \mathrm{ml} \mathrm{Ni-NTA-Agarose}$ slurry (for a culture of 61 ). The lysate-Ni-NTA mixture was incubated for $3 \mathrm{~h}$ at $4^{\circ} \mathrm{C}$ on the rolling incubator. The mixture was filled into $50 \mathrm{ml}$ Falcon tubes and centrifuged at $3000 \mathrm{U}$ for $10 \mathrm{~min}$ (Beckman J6-MI). The supernatant was removed into a bottle (Qiagen) and the $\mathrm{Ni}$ beads were resuspended in a small amount of supernatant and filled into a large column. The filled column was washed with $300 \mathrm{ml}$ of the washing buffer. Finally, Syt1 was eluted with the elution buffer in five fractions $(5 \times 10 \mathrm{ml})$. The eluates were directly mixed with DTT (f.c. $1 \mathrm{mM}$ ). After a rough concentration determination (Bradford) the concentrated fractions were pooled together and mixed with $50 \mu \mathrm{l}$ thrombin $(1 \mathrm{U} / \mu \mathrm{l})$ per $10 \mathrm{ml}$ of volume to cleave the histidine tag. The protein solution was dialyzed in at least 10 times the volume of sample with the desalting buffer at $4^{\circ} \mathrm{C}$ overnight. All the purification steps and the thrombin cleavage itself were checked with SDS-PAGE (Figure 2.2a).

Thrombin and other proteins were furthermore removed with IEXC using a MonoS 5/5 HR column on the Aekta purification system. After loading the protein solution onto the column, elution was programmed as first a prewash with $70 \%$ buffer $\mathrm{A}$ and $30 \%$ buffer B (page 27, Table 2.3 for two column volumes, followed by increasing buffer B's fraction from $30 \%$ to $100 \%$ for five column volumes. Finally the column was washed using 100\% buffer B for two column volumes. All of these eluates were retrieved as sets of separate fractions of $2 \mathrm{ml}$ each. The concentrated fractions were checked using SDS-PAGE (Figure 2.2b). The purification yielded ca. $1 \mathrm{mg}$ protein per 61 of culture. 

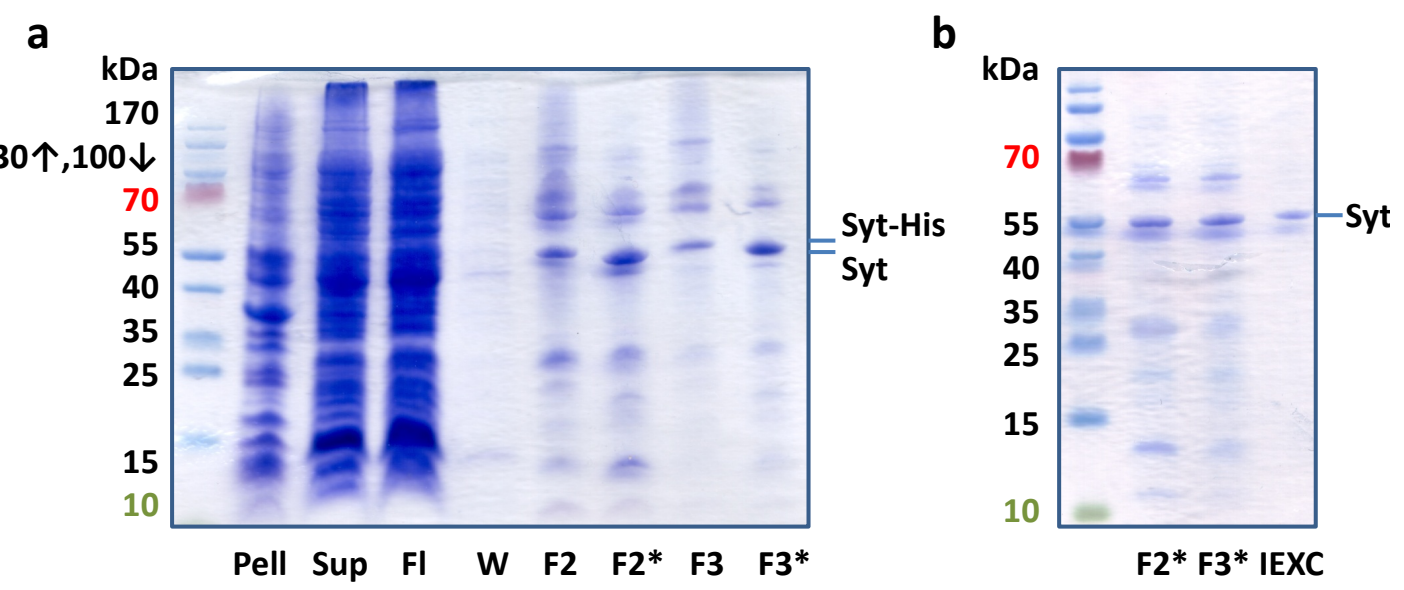

Figure 2.2: Purification of Syt1 with immobilized metal ion affinity chromatography and ion exchange chromatography. a. Purification steps using Ni-NTA beads: the pellet after cell lysis (pell), the supernatant of the lysate (Sup), the flow-through after binding on the Ni-NTA beads (Fl), washing the column with the wash buffer (W), the most concentrated fractions checked with Bradford Kit (F2 and F3) and the proteins after thrombin cleavage and dialysis $\left(\mathrm{F}^{*}\right.$ and F3*). b. Purification with IEXC: protein input $\left(\mathrm{F}^{*}\right.$ and $\left.\mathrm{F}^{*}\right)$ and IEXC output (IEXC). 


\subsubsection{Determination of the protein concentration}

\subsubsection{UV absorption}

The concentrations of the SNARE proteins, Syt1 and $\alpha$-SNAP were determined with UV absorption spectroscopy $(280 \mathrm{~nm})$ [28,88]. The molar extinction coefficient $\varepsilon$ of a protein with amino acids sequence number $n$ could be calculated as Equation 2.1][43]:

$$
\begin{gathered}
\varepsilon_{\text {Protein }}^{280}=n_{\mathrm{Tyr}} \cdot \varepsilon_{\mathrm{Tyr}}^{280}+n_{\mathrm{Trp}} \cdot \varepsilon_{\mathrm{Trp}}^{280}+n_{\mathrm{Cys}} \cdot \varepsilon_{\mathrm{Cys}}^{280} \\
\text { In water: } \quad \varepsilon_{\text {Tyr }}^{280}=1490 \mathrm{mM}^{-1} \mathrm{~cm}^{-1} \\
\varepsilon_{\mathrm{Trp}}^{280}=5500 \mathrm{mM}^{-1} \mathrm{~cm}^{-1} \\
\varepsilon_{\text {Cys }}^{280}=125 \mathrm{mM}^{-1} \mathrm{~cm}^{-1}
\end{gathered}
$$

The concentration of a homogenous protein solution with extinction coefficient $\varepsilon$ could be calculated using Lambert-Beer law (Equation 2.2), in which $c$ is the protein concentration and $d$ is the thickness of the light path.

$$
A b s=\varepsilon \cdot c \cdot d
$$

The protein concentration was measured using a Nanodrop Spectrometer (ND-1000, Thermo Scientific) and the extinction coefficients of the proteins were taken from the program "ProtParam tool" [32].

\subsubsection{Bradford assay}

By protein purification with Ni-NTA beads (page 30, subsection 2.2.1) the concentration of the different fractions of the eluates were checked tentatively with a Bradford Kit (Biorad) [15]. The Bradford assay is a sensitive photometric method for quantitative determination of the protein concentration under a denaturized condition in $\mu \mathrm{g} / \mathrm{ml}$ range. Coomassie brilliant blue G-250 is a triphenylmethane dye which can mainly 
bind to the alkaline amino acids and then turns from its cationic - to its anionic state. The absorption can be observed at $595 \mathrm{~nm}$. To qualitatively test the protein concentra-

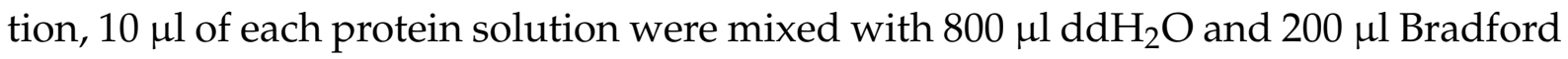
reagent $5 x$ (Biorad, USA).

\subsubsection{Modified Lowry-Peterson protein determination}

For determination of the protein concentration of the SVs, the SV samples were treated according to a modified Lowry-Peterson method [90]. Bovine serum albumin (BSA) was used as the standard in different concentrations in the range of 0 $40 \mu \mathrm{g}$. A series of different dilutions of the SV samples (1:2, 1:5, 1:10 and 1:20) and the standards were filled up with $\mathrm{ddH}_{2} \mathrm{O}$ to an end volume of $1 \mathrm{ml}$. The solutions were incubated with $100 \mu \mathrm{l}$ DOC (Deoxycholic acid, $0.15 \%$ ) for $10 \mathrm{~min}$ at RT. TCA (Trichloroacetic acid, $72 \%$ ) was added to the solutions to precipitate the proteins. The mixtures were incubated for $10 \mathrm{~min}$ on ice and centrifuged for $10 \mathrm{~min}$ at $13000 \mathrm{rpm}$ at $4^{\circ} \mathrm{C}$. The pellets were dissolved and incubated in $750 \mu$ Lowry solution III (page 28 .

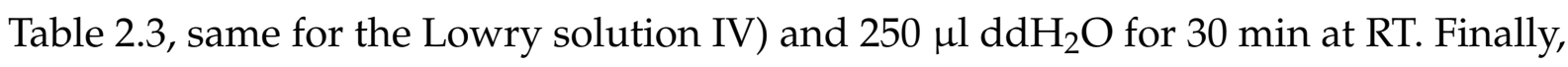
the samples were mixed with $75 \mu$ of Lowry solution IV and incubated for $45 \mathrm{~min}$ at RT. The absorbance was measured at $750 \mathrm{~nm}$ using a Novaspec II photometer (Parmacia Biotech, Germany).This part of the experiment was performed by Dr. Saheeb Ahmed (neurobiology department).

\subsubsection{Determination of Rhodamine Green concentration with UV ab- sorption spectroscopy}

To determine the mouse SVs concentration using FCS, a standard fluorescence dye Rhodamine Green $(\mathrm{RG})$ of known concentration $(10 \mathrm{nM})$ was used to analyze the data by directly comparing particle numbers. RG was solved in PBS (Sigma) and the concentration of RG was determined by UV-absorption spectroscopy (Cary 5E, Varian). The molar extinction coefficient of RG is $\varepsilon_{R G}=75000 \mathrm{M}^{-1} \mathrm{~cm}^{-1}$ at $502 \mathrm{~nm}$ [72]. The 
concentration was calculated using Equation 2.2.

\subsubsection{SDS-PAGE}

The purification steps of proteins were checked using SDS-PAGE (Sodium dodecyl sulfate polyacrylamide gel electrophoresis) [68]. The recipes for the tricine gels are listed in Table 2.5[108]. $3.4 \mathrm{ml}$ separating gel mixture (10\%) was poured between the glass plates ( $0.8 \mathrm{~mm}$ separation) and ca. $1 \mathrm{ml}$ collecting gel mixture was added on top of the separating gel until the chamber was full. A comb with either 10 or 15 wells was inserted directly into the collecting gel solution and could be removed after about $5 \mathrm{~min}$, when the polymerization was finished. The protein samples were mixed with $5 x$ sample buffer (see page 27, Table 2.3, same with the following buffers) and heated to $90^{\circ} \mathrm{C}$ for $10 \mathrm{~min}$. Of each sample, $10 \mu \mathrm{l}$ were loaded into the gel pockets. The gel tank was filled with the cathode buffer in the middle and the anode buffer outside the gel. It took about $25 \mathrm{~min}$ to pull the sample through the collecting gel using 60 Volts and the electrophoresis was promoted at 120 Volts until the blue color of the samples ran out of the gel. For staining, the gel was dipped into the coomassie staining solution, heated for $5 \mathrm{~s}$ in a microwave and rocked for $5 \mathrm{~min}$. To destain the gel, the coomassie staining solution was carefully removed and the gel was incubated in the coomassie destaining solution for another $5 \mathrm{~min}$. The gel could be furthermore destained in water overnight.

\begin{tabular}{l|c|c}
\hline Component & Collecting gel & Separating gel \\
\hline Acrylamide 30\% & $200 \mu \mathrm{l}$ & $1.66 \mathrm{ml}$ \\
Gel buffer & $375 \mu \mathrm{l}$ & $1.68 \mathrm{ml}$ \\
$\mathrm{ddH}_{2} \mathrm{O}$ & $925 \mu \mathrm{l}$ & $570 \mu \mathrm{l}$ \\
Glycerol 50\% & - & $1.06 \mathrm{ml}$ \\
TEMED & $2 \mu \mathrm{l}$ & $3 \mu \mathrm{l}$ \\
APS 10\% & $10 \mu \mathrm{l}$ & $25 \mu \mathrm{l}$ \\
\hline
\end{tabular}

Table 2.5: Tricine (Schägger)-gels. 


\subsubsection{Reconstitution of liposomes}

\subsubsection{Reconstitution of small liposomes}

For the Syt1 tethering experiments, the liposome was reconstituted using size exclusion chromatography as described in earlier papers [105, 138] with the following modifications. All the lipids components were solved in the chloroform/methanol solution (page 25, Table 2.3, the following buffers in this subsection analogously) and mixed with their composition for the liposome. The lipid solution was dried to a lipid film with nitrogen gas or with a rotary evaporator if larger amounts were used. The lipid film was resolved in HPCholate5 buffer to a final lipid concentration of $27 \mathrm{mM}$. $16.7 \mu \mathrm{l}$ lipid mixture were added with protein at a protein:lipid ratio of 1:1000 or 1:750. The lipid protein mixture was filled with HPCholate1.5 buffer to a final volume of $50 \mu \mathrm{l}$. The liposomes were formed by detergent removal using a Sephadex G50 econo column (GE Healthcare, Biorad). The running buffer for the column was HP150. The collected liposome volume was about $250 \mu \mathrm{l}$. The radius of the liposome was about $20 \mathrm{~nm}$ [138]. Figure 2.3 shows a schematic of the size exclusion chromatography. All the lipid compositions used in this study are listed in Table 2.6.

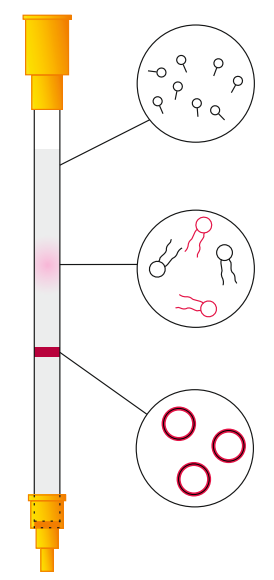

Figure 2.3: Size exclusion chromatography using sephadex G50 econo column to reconstitute small liposomes. The lipid mixture was separated by the sephades 50G column to three phases: the removed detergent (top), the remaining free lipids and proteins (center) and the formed liposomes (bottom).

\subsubsection{Reconstitution of large unilamellar vesicles}

Large unilamellar vesicles (LUVs) were prepared for fusion and docking experiments with purified chromaffin granules (CGs) by reverse phase evaporation and 


\begin{tabular}{l|ccccccc}
\hline Liposome & PC & PE & TRPE & OGPE & PS & Cholesterol & PiP2 \\
\hline TR & 70 & 19 & 1 & 0 & 0 & 10 & 0 \\
TRPS & 50 & 19 & 1 & 0 & 20 & 10 & 0 \\
TRPS5 & 65 & 19 & 1 & 0 & 5 & 10 & 0 \\
TEPS12 & 58 & 19 & 1 & 0 & 12 & 10 & 0 \\
\hline OG & 70 & 18.5 & 0 & 1.5 & 0 & 10 & 0 \\
OGPS & 50 & 18.5 & 0 & 1.5 & 20 & 10 & 0 \\
OGPiP & 49 & 18.5 & 0 & 1.5 & 20 & 10 & 1 \\
\hline
\end{tabular}

Table 2.6: Lipid composition of the liposomes in the Syt1 tethering experiments. Numbers are given in $\mathrm{mol} \%$.

extrusion through polycarbonate membranes with a pore size of $100 \mathrm{~nm}$ [23, 55, 89]. The lipid composition of the liposome was PC:PE:PS:Cholesterol:PI:PIP2:NBDDOPE:Rhodamine-DOPE $=45: 12: 10: 25: 4: 1: 1.5: 1.5$. The size of the LUVs was verified by light scattering. The $\Delta \mathrm{N}$ complex or the 2:1 complex (page 24, Table 2.2) were inserted into the preformed LUVs at a protein:lipid ratio of 1:500 using detergent $n$-octyl- $\beta$-Dglucoside followed by a overnight dialysis [23, 55]. This reconstitution was part of the investigation project of Dr. Yongsoo Park (neurobiology department).

\subsubsection{Purification of mouse and rat SVs}

Purification of mouse and rat synaptic vesicles was described previously [53, 85] and the isolation procedure was optimized by Dr. Saheeb Ahmed in his PhD thesis [1]. This purification protocol allows a one brain isolation of SVs within $24 \mathrm{~h}$ and had an optimized yield and purity.

After differential centrifugation the SVs were separated from the brain and isolated by means of size-exclusion chromatography (Sephacryl S-1000 Superfine HR, GE Healthcare, Germany). Elution was monitored at a wavelength of $280 \mathrm{~nm}$. The purity of the SV isolation was checked by a dotblot assay [64]. The average size of the SVs was 
determined by cryo electron microscopy and the average diameter of the SVs found to be around $40 \mathrm{~nm}$. The purified SVs were eluted using a HB-100 buffer (page 28 , Table 2.3 and the most concentrated fraction was used directly for the FCS measurement (see page 11, subsection 1.5.1) without centrifugation to determine the vesicle concentration. All the mouse and rat SV purifications were performed by Dr. Saheeb Ahmed.

\subsubsection{Purification of chromaffin granules (CGs)}

Purification of CGs was described previously [89]. CGs were separated from the bovine adrenal medullae by differential centrifugation and were isolated and purified with a continuous sucrose density gradient from $0.3 \mathrm{M}$ to $2.0 \mathrm{M}$ (SW41T1 rotor, $27000 \mathrm{rpm}, 1 \mathrm{~h}$ ). The purified CGs were collected from fraction 16 (pellet) and the purity was checked by western blotting [89]. The purified CGs were resuspended in CG buffer (see page 25. Table 2.3). The average diameter of CGs was about $167 \mathrm{~nm}$ determined by the cryo-electron microscopy data. All the CGs were provided by Dr. Yongsoo Park.

\subsubsection{Two-photon confocal fluorescence microscopy setup}

To simultaneously excite two different colors of liposomes, $22 \mathrm{~mW}$ of the output power of a Chameleon titanium-sapphire laser (Coherent, USA) was used for twophoton excitation. The $800 \mathrm{~nm}, 87 \mathrm{MHz}$ laser beam was expanded using a lens system and coupled to an IX71 inverted microscope (Olympus, Germany), reflected to the top of the microscope by a dichroic mirror DC1 (715 DSCPXR, AHF, Germany) and focused by a UPlanSApo 60x/1.2w water immersion objective (Olympus, Germany). The emitted photons passed through the objective and the dichroic mirror DC1.

Scattered light from the excitation beam was blocked by a two-photon rejection filter 2P-SP (E700SP2, AHF, Germany). The emission was collimated using a second lens system and reflected into the detection side of the setup using an ultrabroad band 
dielectric mirror M (650-1130 nm, Newport, USA). The emission was furthermore split by a second dichroic mirror DC2 (590 DCXR, AHF, Germany), filtered in each direction by a bandpass filter BP1/BP2 (HQ 645/75 and HQ 535/50; AHF, Germany) and focused with a lens (either L1 or L2, respectively) to an avalanche photodiode (APD1 or APD2, respectively; SPCM-AQR-13, Perkin-Elmer, Canada; see Figure 2.4 a). The TTL (transistor-transistor-logic) signals from the APD were analyzed using a 4-channel router (PRT 400, PicoQuant GmbH, Germany) and a TCSPC (time-correlated single photon counting) card (TimeHarp200, PicoQuant GmbH, Germany) and saved in PicoQuant's TTTR (time-tagged time-resolved) format. The correlation was processed using a home-made program (provided courtesy Matthias Grunwald).

Figure $2.4 \mathrm{~b}$ shows a schematic of the focus volume for two-photon excitation. The black line indicates the laser beam, and the dark red ellipse marks the excited effective volume $V_{e f f}$ of two-photon excitation (TPE). Only the samples inside $V_{e f f}$ can be excited while the surrounding volume remains dark. The effective volume of two-photon excitation in this study was approx. $0.3 \mathrm{fl}$. 
a

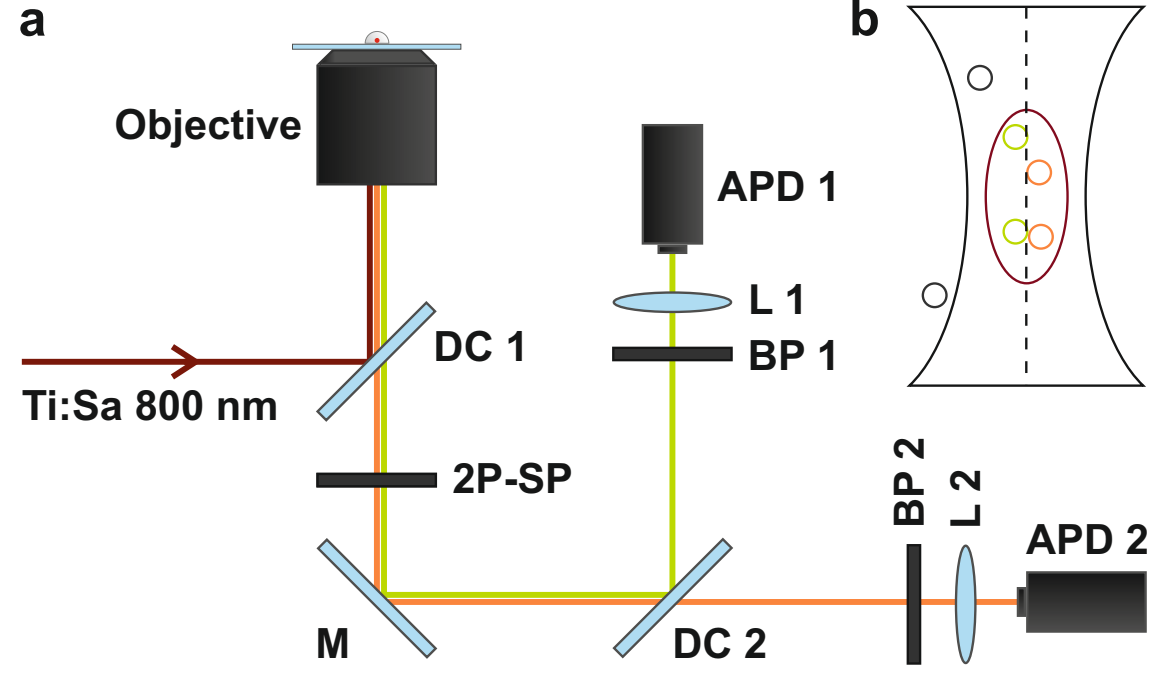

Figure 2.4: Confocal fluorescence microscopy setup for two-photon excitation. a. The pulsed $800 \mathrm{~nm}$ Ti:Sa laser beam is reflected to the objective using a dichroic mirror (DC1) and the emission passes through the objective using the same pathway. The scattered light of the laser beam is blocked by a two-photon rejection filter (2P-SP). The emission light is reflected to the detection side by an ultrabroad band dielectric mirror $(\mathrm{M})$ and split by a second dichroic mirror (DC2) to finally arrive at one of two avalanche photodiodes (APD1 or APD2) after having been filtered by a bandpass filter (BP1 or BP2) and focused by a lens (L1 or L2). b. The dark red ellipse marks the $V_{\text {eff }}$ of the TPE. 


\section{Chapter 3}

\section{Results}

\subsection{Cis- and trans-membrane interaction of synaptotag- $\min -1$}

Synaptotagmin-1 (Syt1) possesses two conserved calcium-binding domains-C2A and $\mathrm{C} 2 \mathrm{~B}-$, which serve as partial calcium coordination sites [37, 95]. The $\mathrm{C} 2$ domains mediate calcium-dependent binding to both SNARE proteins [7, 19, 21, 125] and acidic membrane lipids, with a specific affinity for the membrane lipid phosphatidylinositol4,5-bisphosphate (PiP2) [3, 6, 13, 42, 56, 59, 73, 95, 104, 134]. Separated from the calcium binding pocket, the Syt1 also possesses a poly-basic lysine stretch in C2B domain, which binds to anionic membranes containing phosphatidyl-serine (PS) or PiP2 in a calcium-independent manner [6, 19,73, 104, 134, 141]. The 3D-structure of Syt1 is shown in Figure 1.5 .

However, it is not yet clear how exactly these binding activities contribute to the acceleration of exocytosis [96]. Recent studies suggested that Syt1 may not only bind trans to the plasma membrane but also cis to its own membrane [67, 111]. Using fulllength Syt1 and its mutants, in which either the $\mathrm{Ca}^{2+}$ binding $\mathrm{C} 2$ domains or the $\mathrm{Ca}^{2+}$ independent binding site in $\mathrm{C} 2 \mathrm{~B}$ domain were disrupted, a structural requirement of the cis- and the trans-binding under the influence of PS/PiP2 and SNAREs was dis- 
sected with a sensitive tethering assay based on FCS and FCCS (see page 44, subsection 3.1.1). No SNARE proteins are inserted in the host membrane of Syt1, so that tethering will be observed in a stable state without undergoing fusion.

\subsubsection{Tethering assay based on FCS and FCCS}

The tethering experiments were performed with a two-photon confocal microscope (page 39, subsection 2.2.8) and the data were analyzed using FCS (page 11 , subsection 1.5.1) and FCCS (page 14, subsection 1.5.2) techniques. Figure 3.1 shows a schematic of the tethering experiment data. The different percentages $(0 \%, 50 \%$, and $100 \%$ ) in Figure 3.1 reflect the particle number of tethered liposomes $N_{x}$ (page 15 , equation 1.11) divided by the total number of the green liposomes $N_{g}$ (page 15, equation 1.10):

$$
\text { Tethering }=\frac{N_{x}}{N_{g}} \cdot 100
$$

The result was corrected by substituting a small percentage of the tethering experiment with Syt1-TR and OG liposomes containing no PS or, in the case of the binding assay, the TRPS and OGPS liposomes in HP150 buffer, respectively.

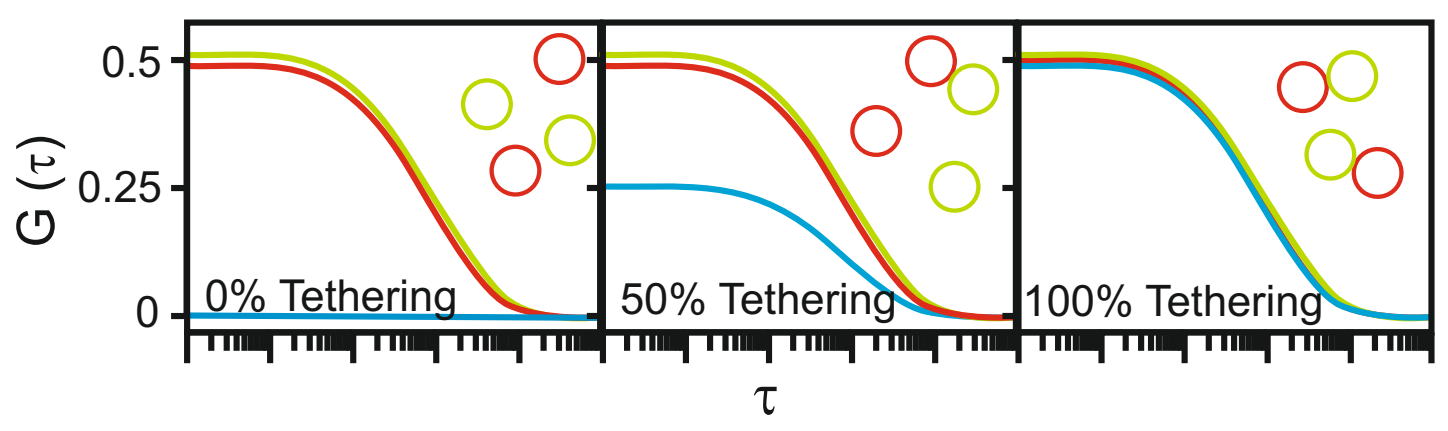

Figure 3.1: Schematic of typical FCCS curves in the tethering assay. Red and green liposomes are in 1:1 ratio. The red and green lines are the FCS measurement for each liposome color and the blue line is the simultaneous FCCS measurement. All three liposome pictures contain two red and two green particles. 
For the experiments, Texas Red (TR) and Oregon Green (OG) labeled small liposomes (page 37, subsection 2.2.5.1) were first measured with FCS at a 1:20 dilution to check their concentrations. Using HP150 buffer (for all buffers see page 25, Table 2.3 the liposomes were diluted to a stock solution with about 100 liposomes in the excitation volume for each color. The tethering experiment was performed in either EGTA buffer or Calcium buffer. The experiment was started directly after mixing (vortex) $90 \mu \mathrm{l}$ buffer and $5 \mu \mathrm{l}$ of each the red and green liposomes at RT. Each droplet $(20 \mu \mathrm{l})$ measurement took $72 \mathrm{~s}$ and the data trace was split into six fragments for the correlation.Each measurement set was repeated at least once with fresh liposomes and buffer. All the results were obtained by averaging of two fresh preparations with the error bar marking the range of the respective data points obtained. Figure 3.2 shows two sets of experimental data, one from an experiment under no-tethering conditions and on from an experiment under full-tethering conditions.
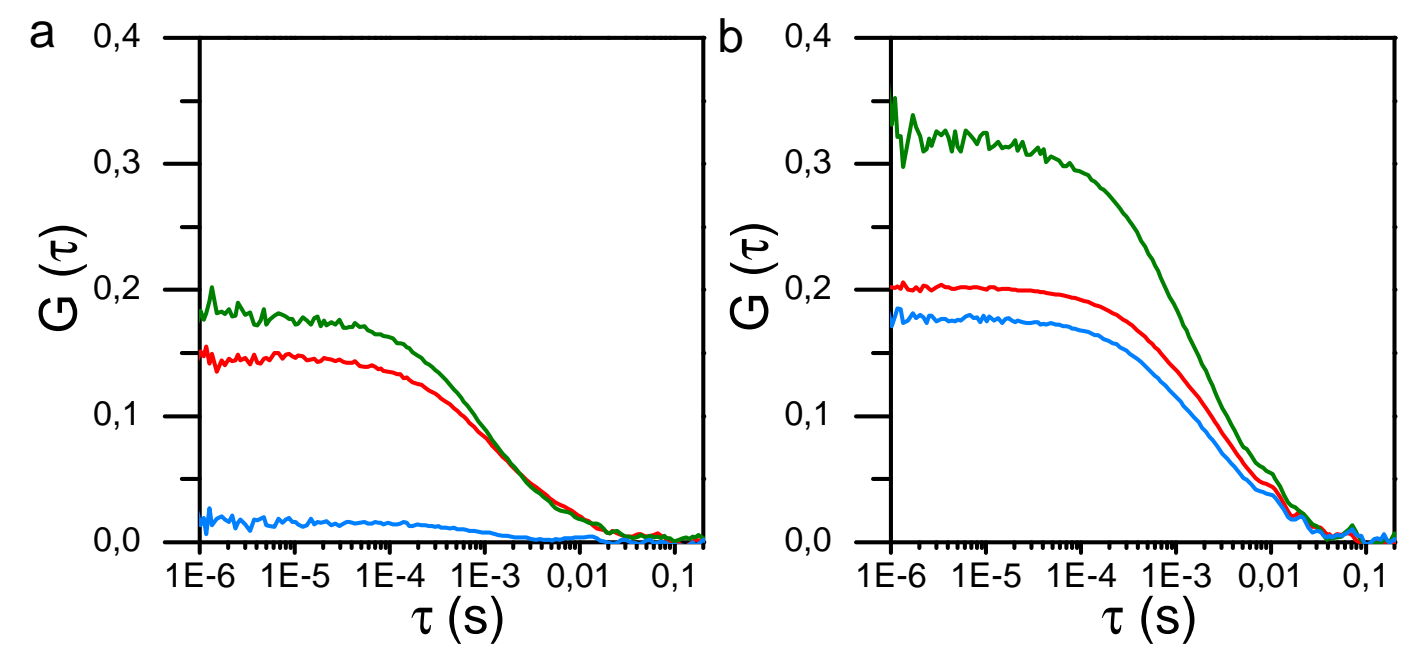

Figure 3.2: Example FCCS data from the tethering experiments. a. Control experiment with OGPS and TR liposomes without Syt in EGTA buffer (no-tethering conditions). b. Tethering experiment with OGPS and Syt1-TR liposomes in calcium buffer (fulltethering conditions). 


\subsubsection{Cis- and trans-interaction of Syt1 to the acidic lipid membrane}

To investigate the membrane interaction of Syt1 (WT), full-length Syt1 and its mutants $\left(\mathrm{a}^{*} \mathrm{~B}, \mathrm{Ab}^{*}, \mathrm{a}^{*} \mathrm{~b}^{*}\right.$ and KAKA) were reconstituted into red TR liposomes in a protein:lipid ratio of 1:1000. Tethering was measured with the protein-free green OG liposomes using FCCS under two-photon excitation. In the $\mathrm{a}^{*} \mathrm{~B}$ and $\mathrm{Ab} \mathrm{b}^{*}$ mutants, either one or both C2 domains $\left(a^{*}, b^{*}\right)$ were disrupted, so that one or both C2 domains cannot bind calcium and thus to the acidic phospholipids. In the KAKA mutant, the polybasic stretch of the C2B domain was mutated by two of the lysine residues, and the mutant cannot bind to PiP2 or PS without presence of calcium. Figure 3.3 shows the domain structures of Syt1 and the mutants used.

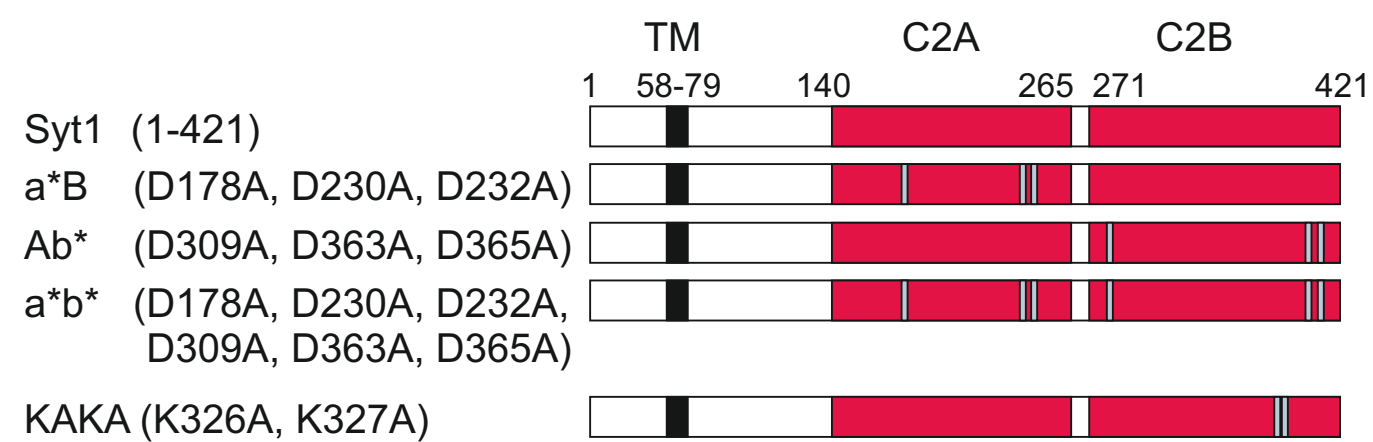

Figure 3.3: Domain structures of Syt1 and its mutants used in this experiment.

The TR liposomes contain $1 \mathrm{~mol} \%$ TRPE and either $0 \mathrm{~mol} \%$ (TR) or $20 \mathrm{~mol} \%$ PS (TRPS) whereas all the OG liposomes contain $1.5 \mathrm{~mol} \%$ OGPE and $20 \mathrm{~mol} \%$ PS (OGPS). One population of the OG liposomes was reconstituted with an additional $1 \mathrm{~mol} \%$ PiP2 (OGPiP). All of the liposome compositions are listed in Table 2.6, on page 38

The first set of experiments was tethering between the Syt1 (WT) bearing TR liposomes and protein-free OGPS liposomes in either the absence or the presence of $100 \mu \mathrm{M} \mathrm{CaCl}_{2}$ [111]. A tethering efficiency of $73 \%$ was observed with calcium whereas the tethering was reduced to $20 \%$ without calcium (Figure 3.4 , WT). The experiments were repeated with mutants of Syt1. $\mathrm{a}^{*} \mathrm{~B}$ and $\mathrm{Ab}^{*}$ showed similar results as the WT, 
i.e. calcium almost doubled the tethering to more than $85 \%$. The double mutant $a^{*} b^{*}$ did not show a calcium dependence, whereas the KAKA mutant tethered the OGPS liposome exclusively in the presence of calcium, as expected [3, 60, 141] (Figure $3.4 a, a^{*} B, A b^{*}, a^{*} b^{*}$ and KAKA). In agreement with previous observations, the calcium-independent tethering is mediated by the polybasic lysine stretch of the $\mathrm{C} 2 \mathrm{~B}$ domain [3, 141]. In all experiments with mutants, a small increase in tethering was observed compared to the respective WT experiment under the same conditions. One possible explanation for this increase is an enhanced membrane interaction due to the removal of charges.

The KAKA mutant possesses only calcium-dependent binding sites and the binding was inhibited when calcium was chelated with $500 \mu \mathrm{M}$ EGTA. To check the binding specifity of the calcium, the tethering experiment with the KAKA mutant was repeated with an extra $1 \mathrm{mM}$ ATP $/ \mathrm{MgCl}^{2}$. $\mathrm{Mg}^{2+}$ did not influence membrane tethering. A control experiment was performed with protein-free TR liposome. No tethering was observed in both the absence and presence of calcium, which indicated that the tethering was dependent on Syt1 only (Figure 3.4a, Control). Fusion was monitored by lifetime analysis of OG. Because TR and OG form a FRET pair (page 17, subsection 1.5.4][23]), a reduction of the OG lifetime indicates lipid mixing of the liposomes. No major change in lifetime was observed and thus it was concluded that fusion did not occur.

To analyze whether PiP2 enhances membrane tethering, the trans-tethering experiments described above were repeated using OGPiP liposome as target liposome (Figure 3.4 ). No major differences were observed in all these tethering experiments. According to a previous study, a high percentage of PS could reduce the PiP2 effect in the binding assay between the labeled Syt1 and the labeled liposomes [95].

To investigate whether cis-binding of Syt1 to its own membrane affects its tethering activity, the entire tethering experiments in Figure $3.4 \mathrm{a}$ and b were repeated using Syt1 bearing the TRPS liposome instead of the TR liposome. Most strikingly, the presence of $20 \%$ PS almost completely inhibited the membrane tethering under all conditions (Figure 3.4 . and $\mathrm{d}$ ). 
To examine the the lower boundary of this cis-binding effect, the PS percentage in the host liposomes was lowered to $12 \%$ (TRPS12) and 5\% (TRPS5). $12 \%$ PS is the typical PS composition in a native rat synaptic vesicle [119]. Similarly, cis-preventing was observed in the tethering experiment with Syt1 WT (Figure 3.5a). The cis-binding could finally be inhibited using 5\% PS in TR liposome in the presence of calcium or PiP2 (Figure 3.5b). Here, the calcium-dependent trans-binding was recovered to the maximum binding rate, whereas the calcium independent tethering only appeared if the target membrane contained $1 \%$ PiP2.

These unexpected results suggested a different binding model between Syt1 and liposomes compared with the findings of previous studies, which had shown that soluble $\mathrm{C} 2 \mathrm{AB}$ domains were able to cluster liposomes containing acidic phospholipids in the presence of calcium $[3,60,141]$. When comparing these results, it is conceivable that membrane anchorage reduces the mobility of the $\mathrm{C} 2$ domains in such a way that cisbinding alone already exhausts both binding sites of $\mathrm{C} 2 \mathrm{~A}$ and $\mathrm{C} 2 \mathrm{~B}$, and that therefore no $\mathrm{C} 2$ domain is available for trans-binding. To shed light on this issue, we carried out further tethering experiments using a soluble $\mathrm{C} 2 \mathrm{AB}$ domain (97-421). These results are laid out in the next subsection.

Figure 3.4: Liposome tethering mediated by membrane-anchored Syt1. Tethering was measured using TP-FCCS in the absence (black) or the presence (red) of $100 \mu \mathrm{M} \mathrm{CaCl}_{2}$. The donor liposomes contain either $0 \%$ or $20 \%$ PS, while the acceptor liposomes contain either 20\% PS or both 20\% PS and 1\% PiP2. a. Tethering experiment using wild-type Syt1 (WT) and its mutants. Except for the $\mathrm{a}^{*} \mathrm{~b}^{*}$ mutant, calcium drastically increases the tethering rate in all the other cases. The KAKA mutants bind acceptor vesicles only in the presence of calcium. b. Tethering experiment in the presence of PiP2 in the acceptor vesicle confirming the results in the first set of the experiments (a). c. Cis-binding mediated by PS in the donor vesicle. Tethering was observed neither for Syt1 nor for any of its mutants. d. Cis-binding in the presence of PiP2 in the acceptor vesicle could still be observed. All control experiments were performed with protein-free donor vesicles. 

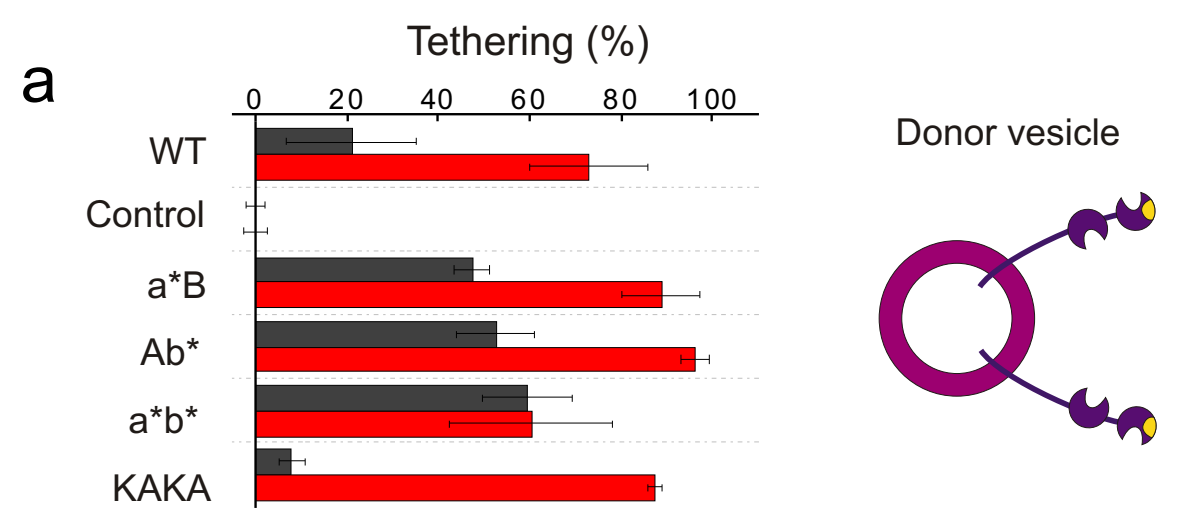

Acceptor vesicle

b
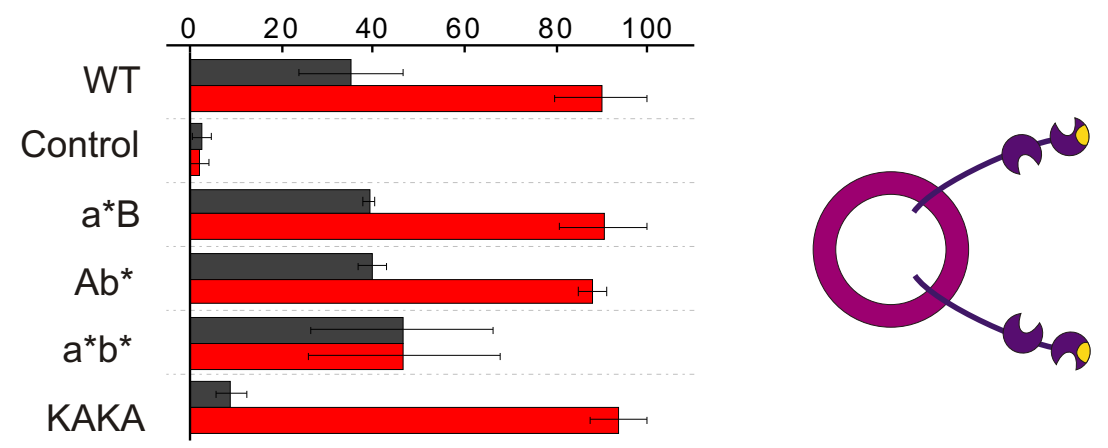

C

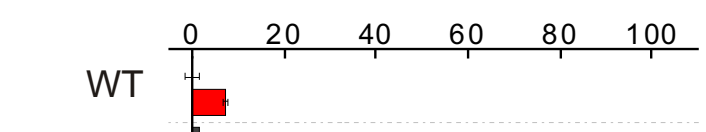

Control
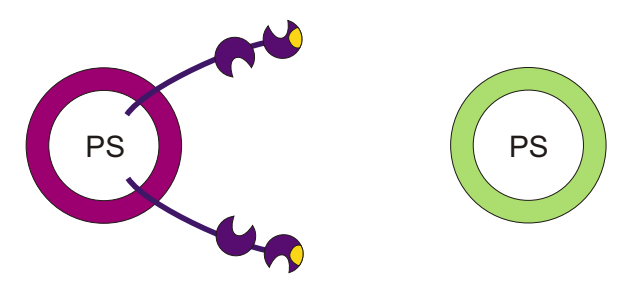

d

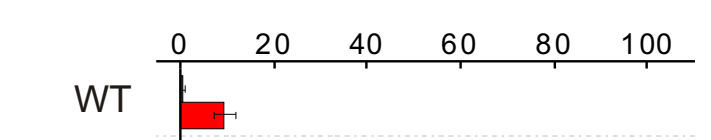

Control
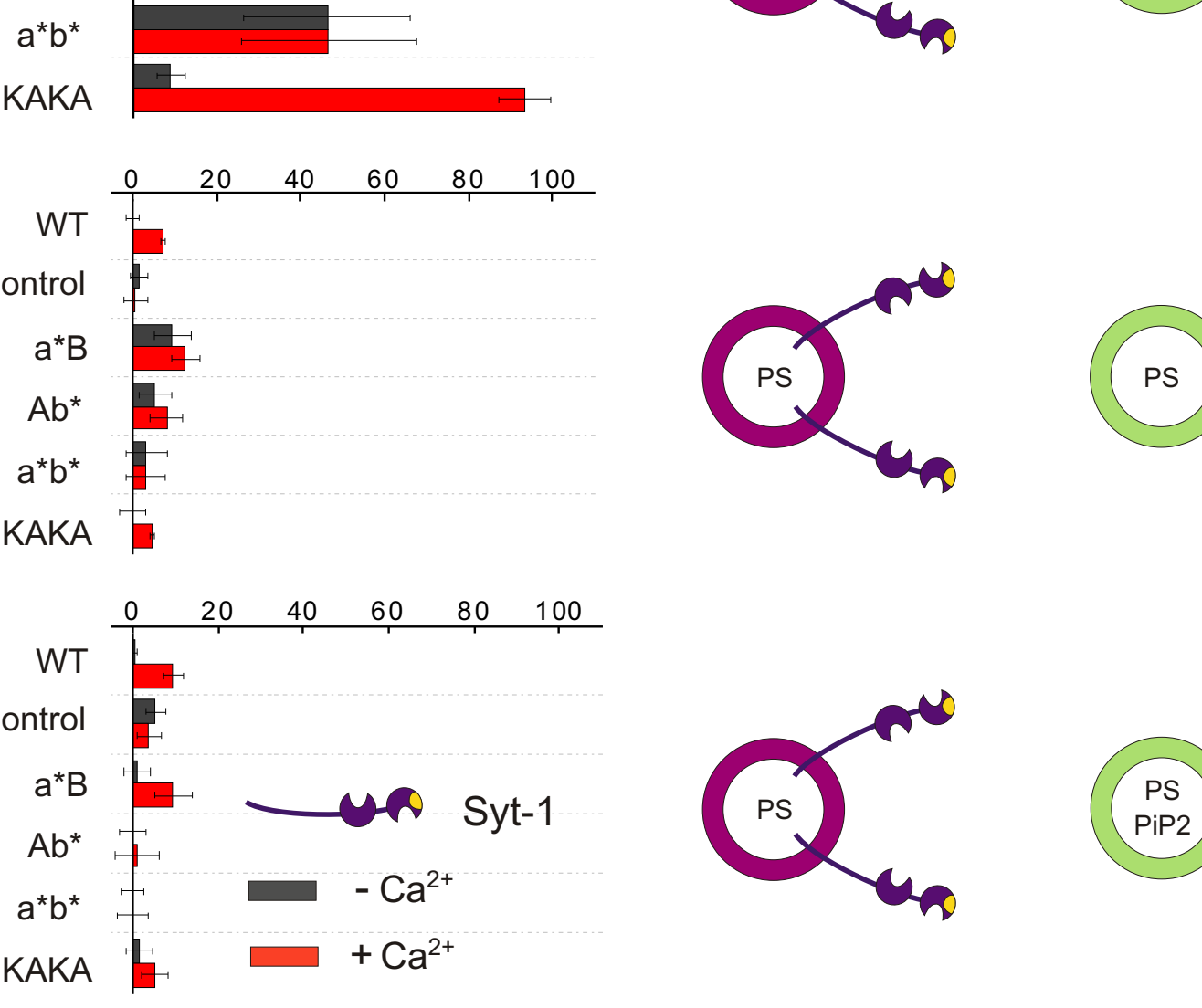

Control: No Synaptotagmin in donor vesicle 

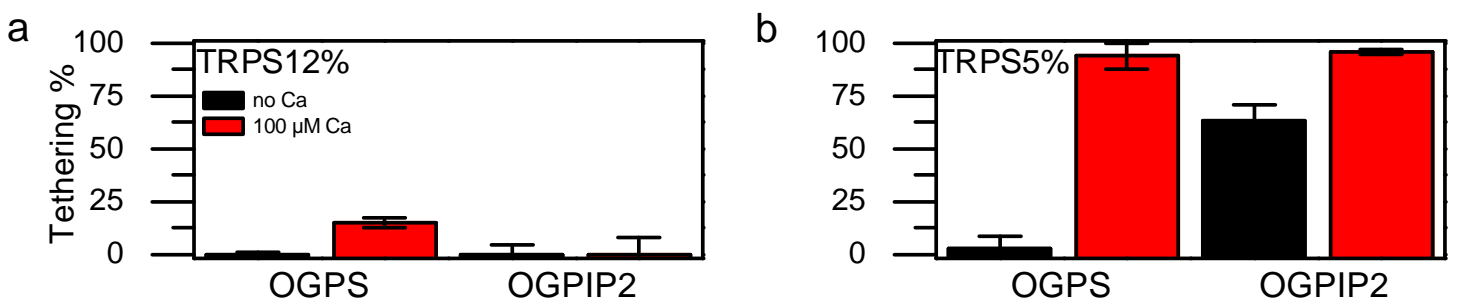

Figure 3.5: Syt1 mediated membrane tethering in the presence of $12 \%$ and of $5 \%$ PS in the cis-membrane. Tethering was measured in the absence (black) and the presence (red) of $100 \mu \mathrm{M} \mathrm{CaCl}_{2}$. a. $12 \%$ PS prevented tethering under all conditions. b. With 5\% PS in the Syt1 liposome membrane, tethering required either the presence of calcium or PiP2 in the target membrane.

\subsubsection{The soluble C2AB domain of Syt1 is able to cluster liposomes at a saturating concentration}

The C2AB (97-421) fragment and its single cysteine mutant AF-C2AB labeled with alexa fluor 488 were used to cross-link the TR and OG liposomes containing 20\% PS. The domain structures are shown in Figure 3.6 .

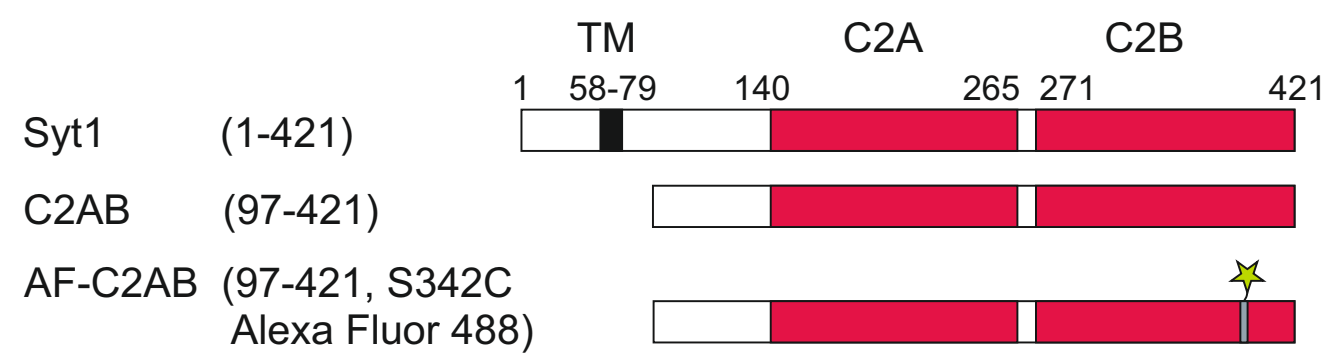

Figure 3.6: Domain structures of the soluble C2AB domain and its Alexa Fluor 488 labeled mutant.

Different $\mathrm{C} 2 \mathrm{AB}$ dilutions ranging from $50 \mathrm{nM}$ to $860 \mathrm{nM}$ were used to cluster the green and red liposomes under $100 \mu \mathrm{M}$ calcium. All experiments were started by adding the $\mathrm{C} 2 \mathrm{AB}$ domain into the liposome mixture. Clustering could be observed only upon addition of elevated $\mathrm{C} 2 \mathrm{AB}$ concentrations. The first evident clustering was determined with $215 \mathrm{nM}$ C2AB (Figure 3.7). No clustering was observed below this 
concentration even after $30 \mathrm{~min}$ of incubation. The clustering was reversible by adding 2 mM EGTA after clustering. The experiments with either EGTA or calcium alone, as well as with C2AB in EGTA buffer are shown as negative controls (Figure 3.7).

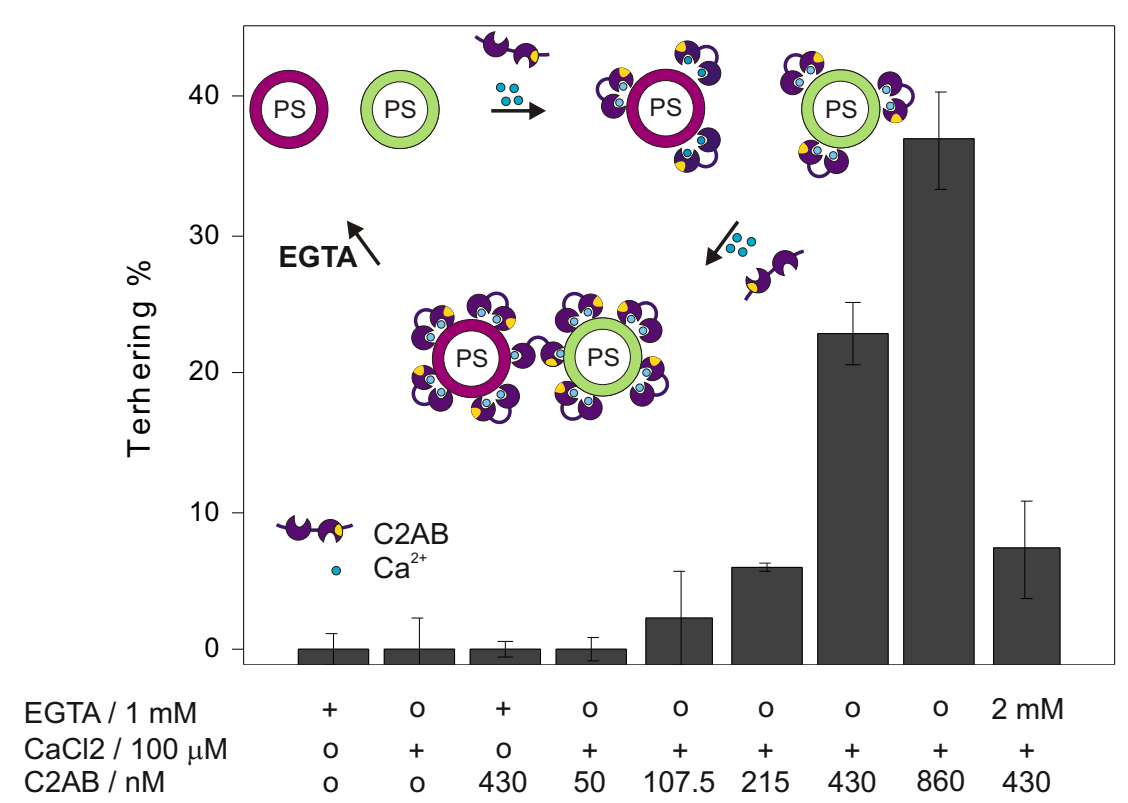

Figure 3.7: Clustering of liposomes mediated by soluble C2AB domain. Columns 1-3: negative controls. Columns 4-8: clustering experiment using soluble $\mathrm{C} 2 \mathrm{AB}$ domain of Syt1 of the concentration from $50 \mathrm{nM}$ to $860 \mathrm{nM}$. Column 9: clustering mediated by $430 \mathrm{nM}$ C2AB could be reverted using 2 mM EGTA.

It is essential to determine the concentration of soluble $\mathrm{C} 2 \mathrm{AB}$ relative to the liposome required to cross-link the liposomes and to find the reason for the absence of clustering at low concentrations. A binding experiment was performed in which Alexa Fluor 488 labeled $\mathrm{C} 2 \mathrm{AB}$ fragments (AF-C2AB) were added to the TRPS liposome containing 20\% PS (Figure 3.8 ) and the binding was measured with FCCS, which is capable of monitoring free and bound liposomes separately under the same condition. The lowest non-clustering concentration $(50 \mathrm{nM})$ and the lowest clustering concentration (215 nM) of AF-C2AB were added to the TRPS liposome (Figure 3.8 $)$ ). Both experiments resulted in a high efficiency of soluble AF-C2AB binding to the liposome. 
All the results were calculated as the ratio of bound liposome relative to the entire liposome. At $215 \mathrm{nM}$, when clustering began to be observable, all the liposomes were bound with AF-C2AB. The binding could be reversed by adding 1 mM EGTA.

a
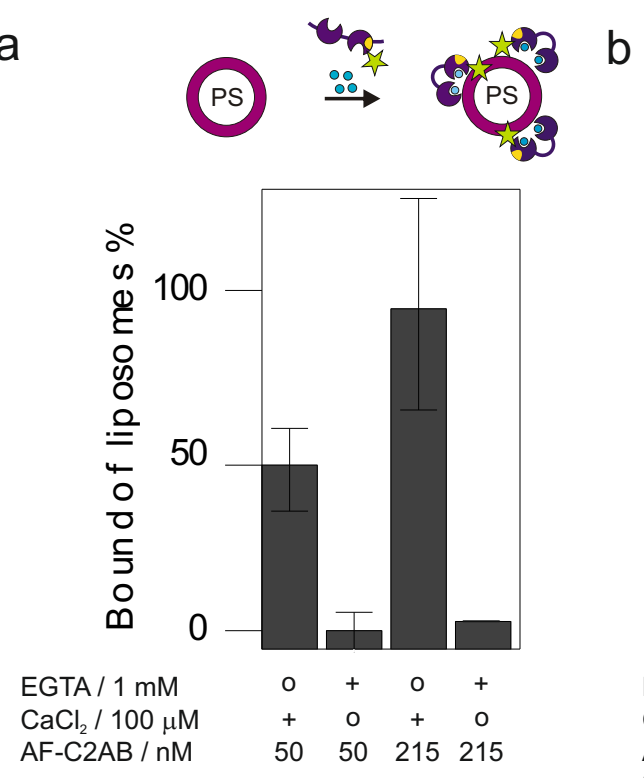

b
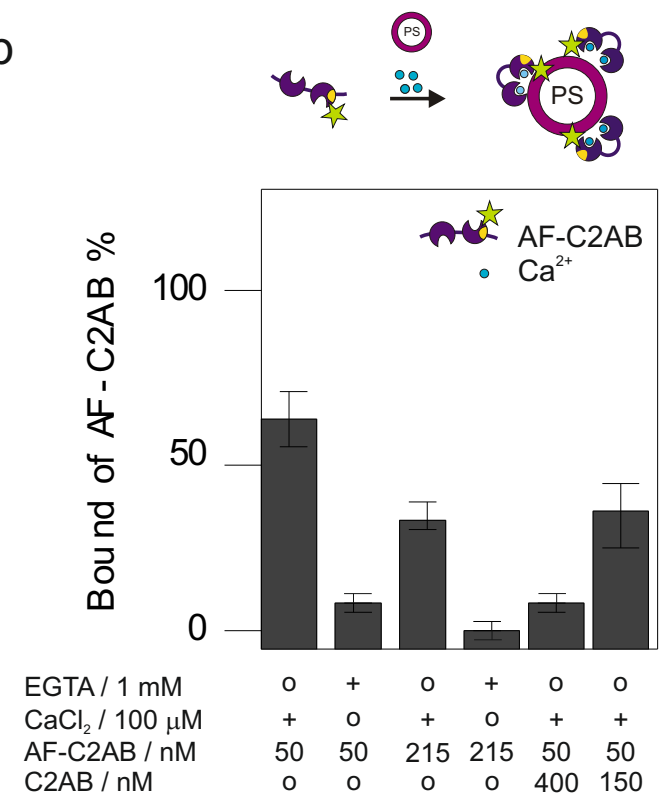

Figure 3.8: Binding assay of soluble C2AB labeled with alexa fluor 488 to TRPS liposome containing $\mathbf{2 0} \%$ PS. Binding was measured by TP-FCCS. a. Calculated as fraction of the bound liposomes with AF-C2AB. b. Columns 1-4, calculated as fraction of bound AF-C2AB fragments with liposome. b. Columns 5-6, binding was competed by unlabeled $\mathrm{C} 2 \mathrm{AB}$ fragments in two different concentrations.

The difference between calcium-dependent clustering and binding indicates that a saturation of binding could be necessary to achieve clustering. To verify this finding, the data from the very same experiments were used to calculate the fraction of bound $\mathrm{AF}-\mathrm{C} 2 \mathrm{AB}$ fragments among the total number of AF-C2AB fragments (Figure 3.8p). Contrary to the liposome binding rate, more than $60 \%$ of the fragments were bound to the liposomes at $50 \mathrm{nM}$ AF-C2AB whereas only $30 \%$ binding rate was achieved at $215 \mathrm{nM}$. The binding was calcium-dependent and could be inhibited with $1 \mathrm{mM}$ EGTA (Figure 3.8p, columns 1-4).

From this set of FCCS data, the fraction of bound liposomes and AF-C2AB, as 
well as the fraction of free components can be read out easily. With these, the binding constant $K_{D}$ between liposomes $([A])$ an AF-C2AB $([B])$ can be calculated. The binding kinetics can be described by:

$$
A+B \leftrightharpoons A B
$$

and the binding constant $K_{D}$ is then calculated as:

$$
K_{D}=\frac{[A]_{\text {free }} \cdot[B]_{\text {free }}}{[A B]}
$$

The total number of liposomes $[A]_{0}$ is a sum of the bound liposomes $[A B]$ and the free liposomes $[A]_{\text {free }}$. The fraction of bound liposomes can be calculated as:

$$
\frac{[A B]}{[A]_{0}}=\frac{[B]_{\text {free }}}{K_{D}+[B]_{\text {free }}}
$$

In this equation, $\frac{[A B]}{[A]_{0}}$ can be taken directly from the fraction of bound liposomes in Figure 3.8 ("Bound \% of liposome"), and $[B]_{\text {free }}$ can be calculated with the AF-C2AB concentration and the fraction bound AF-C2AB in the Figure $3.8 \mathrm{p}$ ("Bound \% of AF$\left.\mathrm{C} 2 \mathrm{AB}^{\prime \prime}\right)$. Using these data, the binding constant $K_{D}$ can be calculated as in Table 3.1 .

\begin{tabular}{l|l|l|l|l}
\hline $\begin{array}{l}{[B]_{0}} \\
(\mathrm{nM})\end{array}$ & $\frac{[A B]}{[A]_{0}}$ & $\frac{[A B]}{[B]_{0}}$ & $\begin{array}{l}{[B]_{\text {free }}} \\
(\mathrm{nM})\end{array}$ & $\begin{array}{l}K_{D} \\
(\mathrm{nM})\end{array}$ \\
\hline 50 & 0.5 & 0.6 & 20 & 20 \\
215 & 0.9 & 0.3 & 150.5 & 16.7 \\
\hline
\end{tabular}

Table 3.1: Binding constant between liposomes and AF-C2AB fragments.

The average $K_{D}$ is about $18 \mathrm{nM}$. With Equation 3.4 , the binding kinetics between liposomes and AF-C2AB can be computed (Figure 3.9).

Addition of $215 \mathrm{nM}$ AF-C2AB, which is 10-fold over the $K_{D}$ value, lead to a concentration of $150.5 \mathrm{nM}$ free AF-C2AB fragments after binding to the liposomes. According to the binding kinetics, this high concentration is already within the saturation range of the AF-C2AB. This was also confirmed by the binding experiments using extra amounts of the unlabeled $\mathrm{C} 2 \mathrm{AB}$ fragments, in addition to the $50 \mathrm{nM}$ AF-C2AB. 


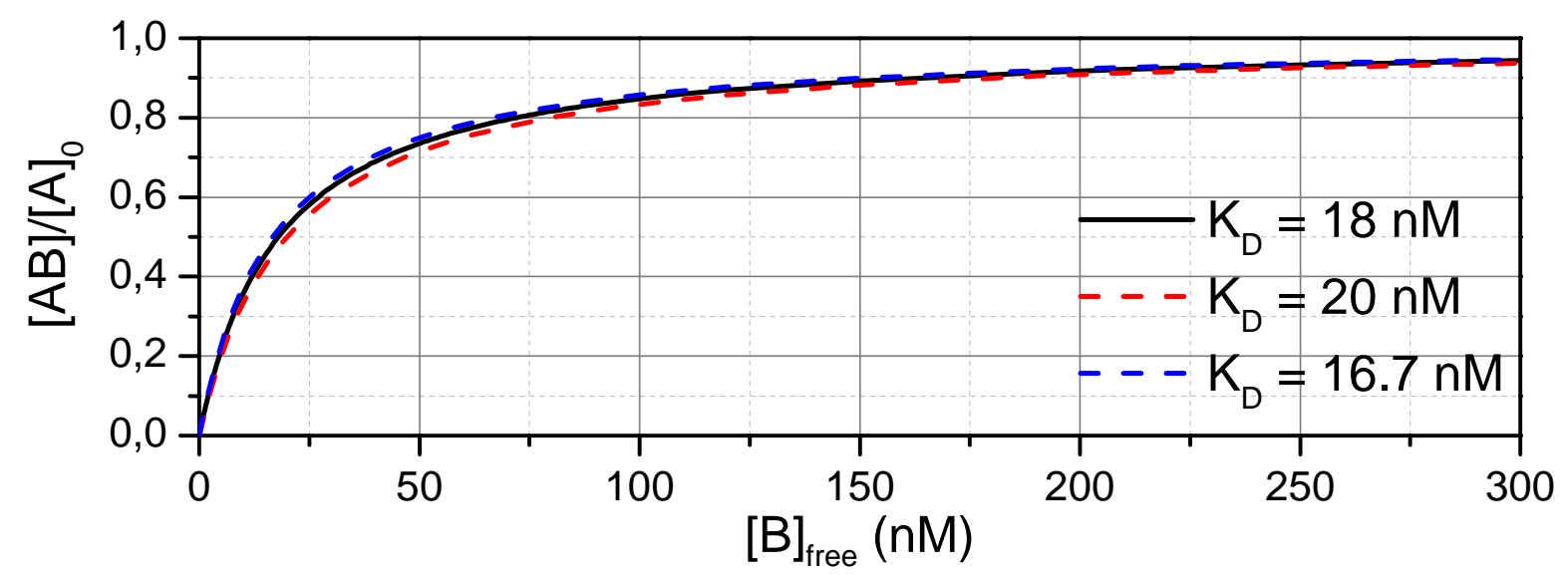

Figure 3.9: Binding kinetics between liposomes (A) and AF-C2AB (B). $[A B]$ is the concentration of bound liposomes with AF-C2AB fragments. $[A]_{0}$ is the total concentration of free and bound liposomes. The fraction of bound liposomes $\frac{[A B]}{[A]_{0}}$ can be read off from Figure 3.8 a. $[B]_{\text {free }}$ is the concentration of free AF-C2AB fragments, which can be calculated as in the Table 3.1 . $K_{D}$ is the binding constant between liposomes and AF-C2AB fragments.

$400 \mathrm{nM}$ C2AB substantially competed with AF-C2AB and resulted in a binding rate below $10 \%$, whereas additional $150 \mathrm{nM} \mathrm{C} 2 \mathrm{AB}$ reduced the binding rate to $35 \%$, similar as the binding rate using $215 \mathrm{nM}$ AF-C2AB alone (Figure 3.8p, columns 5-6). In conclusion, soluble $\mathrm{C} 2 \mathrm{AB}$ fragment clusters liposomes in a calcium-dependent manner and requires the saturation of the binding sites on the membrane surface.

\subsubsection{Syt1-SNARE interaction mediated membrane tethering}

To investigate the membrane tethering mediated by Syt1-SNARE interaction [7, 19, 21.125] Syt1 and SNARE proteins were reconstituted separately into TR and OG liposomes. One TR liposome population contained 20\% PS to check whether back binding is preferred compared to the Syt1-SNARE interaction. All the SNAREs bearing OG liposomes contained no acidic lipids, so that the trans-tethering of Syt1 to PS or PiP2 was ruled out. Three different SNARE combinations were used in this set of experi- 
ments: Sx1A (syntaxin-1A, 183-288) alone, a binary 2:1 complex Sx1A-SN25 (syntaxin 1A, 183-288 and SNAP-25, 1-206) and a fully assembled ternary complex Sx1A-SN25Sb2 (synaptobrevin 2, 1-96, SNAP-25, 1-206 and syntaxin 1A, 183-288). The ternary complex was reconstituted by incubating 2:1 complex and $\mathrm{Sb} 1-96$ in a ratio of $1: 2$ at $4^{\circ} \mathrm{C}$ over night, and the mixture was furthermore used for liposome reconstitution. Thus the liposomes of the ternary complex also contained single Sx1A. The protein:lipid ratio for Syt1 was 1:750 and for SNAREs it was 1:1000. All the experiments were performed in either EGTA or $100 \mu \mathrm{M}$ calcium buffers.

Efficient tethering of about $40 \%$ was observed when Syt1 bearing liposome lacked PS. The tethering was calcium independent except when using Sx1A alone. This result agrees with the findings of previous studies [7, 19, 21, 125], showing that the interaction between Syt1 and Sx1A is enhanced by calcium (Figure 3.10 a). Two negative controls were measured using protein-free OG liposomes under the same conditions and with an additional $1 \mu \mathrm{M}$ free SNAP25 in the buffer, respectively, to exclude the unspecific tethering caused by SNAP25 (Figure 3.10a, control).

The background tethering measured using TR-liposomes lacking Syt1 was significantly lower than in the tethering experiment (Figure 3.10p), indicating that tethering is mediated by Syt1-SNAREs interaction. The tethering was Syt1-SNARE specific, since the presence of $10 \mathrm{mgl}^{-1} \mathrm{BSA}$ could not prevent it. Similarly to subsection 3.1.2. tethering was missing completely when $20 \%$ PS was present in the Syt1 liposome (Figure 3.10 c).

Since the Syt1-SNARE mediated membrane tethering mainly proceeded in a calcium-independent manner, the question arises whether this tethering is caused by the polybasic stretch of the $\mathrm{C} 2 \mathrm{~B}$ domain of Syt1. Therefore, all the tethering experiments were repeated using KAKA in which only the calcium-dependent binding sites C2A and $\mathrm{C} 2 \mathrm{~B}$ are active (Figure $3.10 \mathrm{~d}$ and e). Both tetherings mediated by SNARE complexes were reduced to the same levels as the non-tethering Syt1 (Figure 3.10 d). The calcium enhancement of the tethering mediated by single Sx1A was preserved, but the tethering rate was slightly reduced as compared to the Syt1 WT experiment (Fig- 
Tethering (\%)

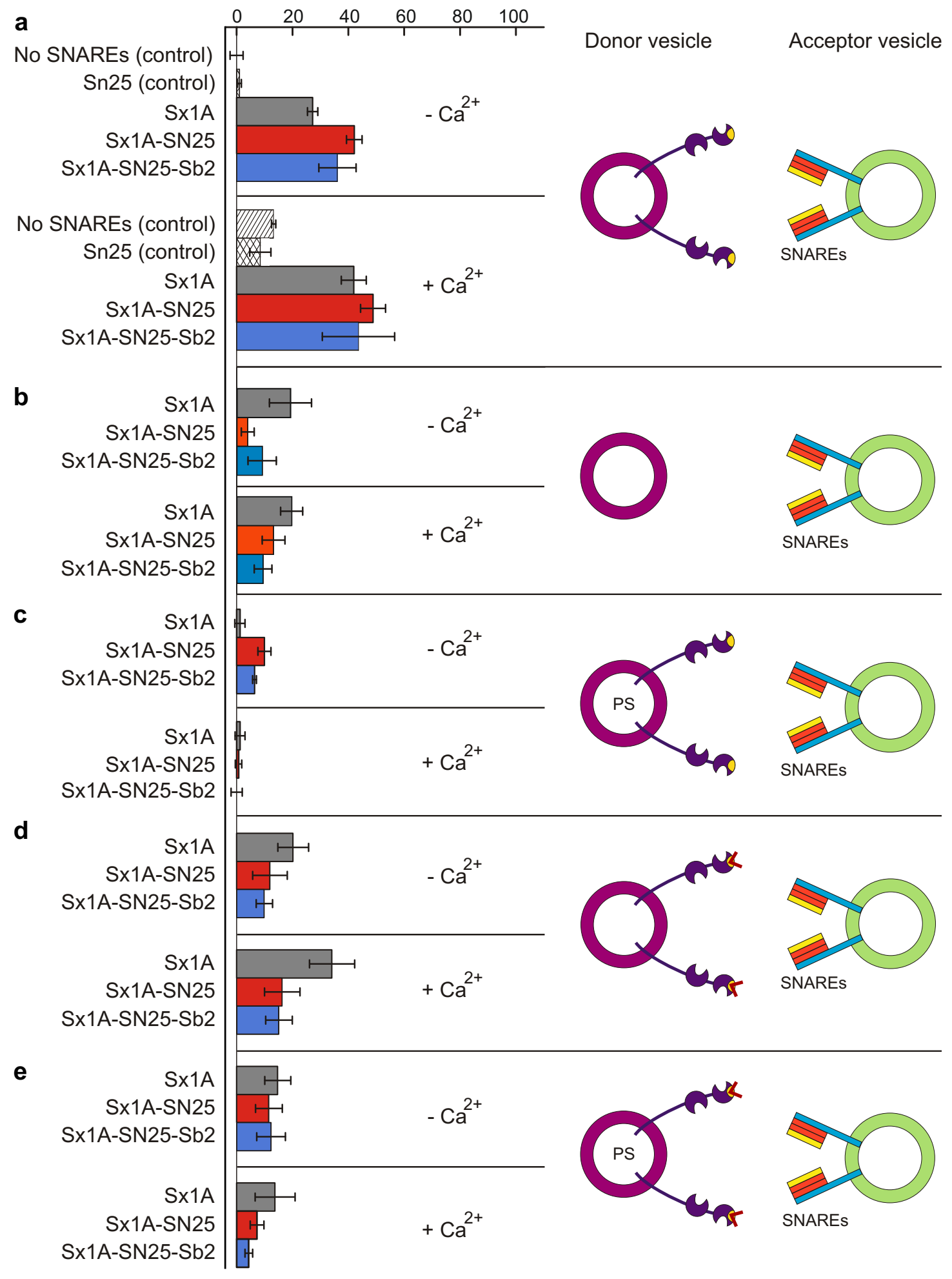


Figure 3.10: Tethering of liposomes mediated by membrane-anchored Syt1-SNAREs interactions. Donor vesicles containing either $0 \%$ or $20 \%$ PS were reconstituted with Syt1 WT or its KAKA mutant $(P: L=1: 750)$. Acceptor vesicles free of PS were reconstituted with purified recombinant Sx1A (gray), a binary 2:1 complex Sx1ASN25 (red), or a ternary SNARE complex Sx1A-SN25-Sb2 (blue, P:L = 1:1000). Control measurements were performed either without SNARE or with $1 \mu \mathrm{M}$ soluble SNAP-25. Tetherings were carried out in the absence $\left(-\mathrm{Ca}^{2+}\right)$ or presence $\left(+\mathrm{Ca}^{2+}\right)$ of $100 \mu \mathrm{M} \mathrm{Ca} 2+$. a. Tethering was observed under no PS condition. $\mathrm{Ca}^{2+}$ dependency was observed only by acceptor vesicle with Sx1A. b. Background tethering without Syt1 as a control. c. No tethering was determined when Syt1 liposomes contained 20\% PS which indicated back binding of Syt1. $\mathrm{d}$ and e. Repeated tethering and back binding measurements with KAKA mutant and the determined tetherings reduced to the background level except with Sx1A liposomes which still showed a $\mathrm{Ca}^{2+}$ dependency.

ure $3.10 \mathrm{~d})$. Presence of PS in the Syt1 liposome significantly reduced the tethering with single Sx1A to the level of the Syt1-lacking background (Figure 3.10). This KAKA mutant experiment was performed by Sabrina Schroeder and Chao-Chen Lin.

All the results reported in this section were summarized in a recent publication [123].

\subsection{Characterization of mouse synaptic vesicles by aver- age protein mass}

Neuronal exocytosis requires fusion of synaptic vesicles (SVs) with the presynaptic membrane. Characterization of the SVs in terms of protein contents and lipid composition as well as SNARE-mediated membrane fusion and neurotransmitter uptake is essential to understand the fusion process and the storage of neurotransmitters. Biological and physical characterization of the rat SVs was done by Takamori et al. in 
2006 [119]. A detailed picture of the SVs including the quantified mass, composition and copy number of the respective proteins and lipids as well as the size and density per single vesicle was obtained (Figure 3.11).

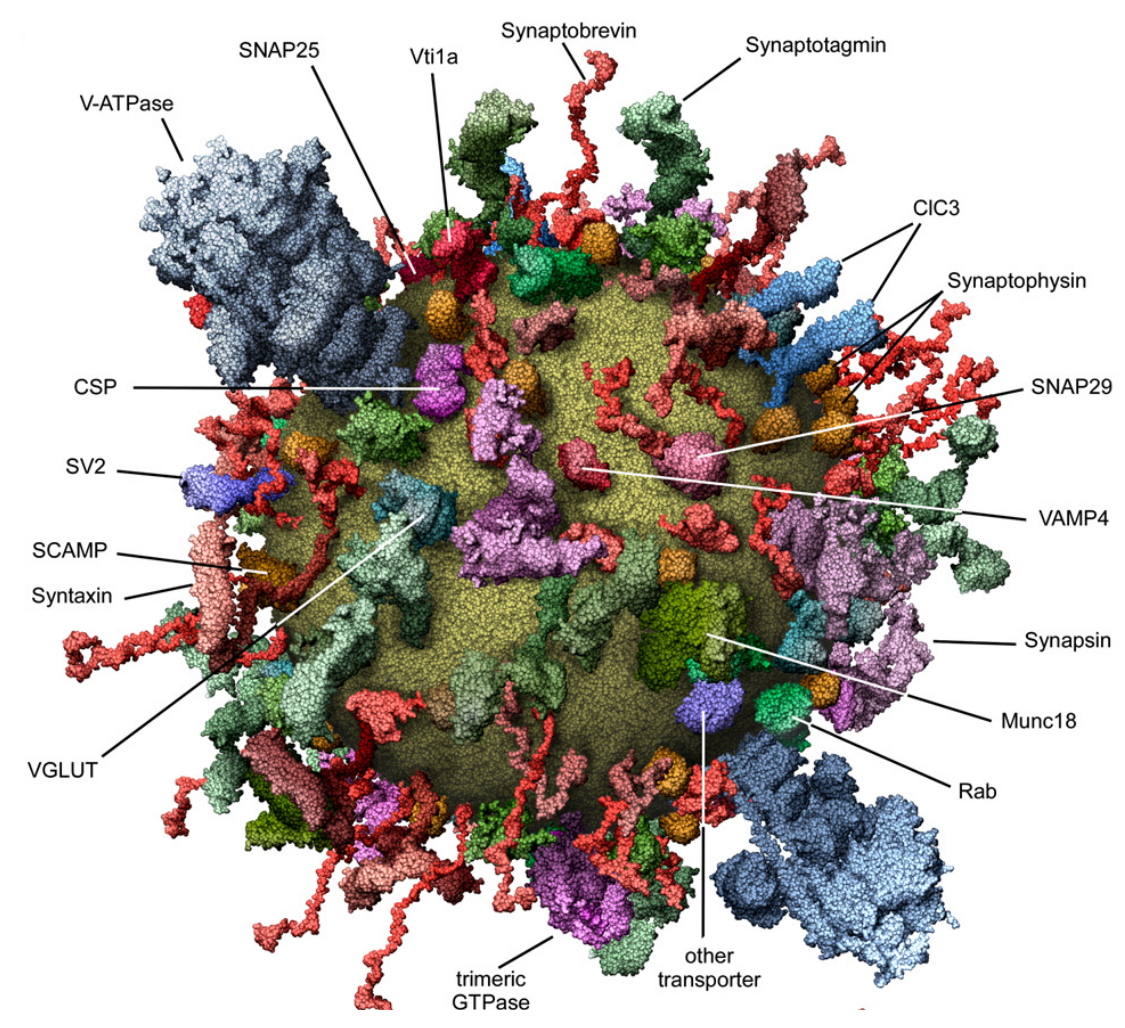

Figure 3.11: Molecular model of an average SV. [119]

The mouse is one of the standard species of animal experiments and shares common ancestry with the rat. Thus, it is a natural extension of the previous study, to characterize the protein and lipid structures of the mouse SVs at a single vesicle level. In the following subsections, a vesicle counting assay based on TP-FCS is described to determine the SV particle concentration, which is essential for characterizing all the biological properties of a single vesicle. Furthermore, combining the protein concentrations determined by means of a modified Lowry-Peterson method, an average mass of proteins per mouse SV compared to that of the rat could be calculated. 


\subsubsection{Synaptic vesicle labeling with FM 1-43 dye}

FM dyes are widely used as outer membrane labeling dyes in monitoring endoand exocytosis, vesicle trafficking, and vesicle fusion in various systems [2, 12, 16, 54, 124]. FM 1-43 and its derivatives harbor a water-soluble cationic head group and a membrane permeable hydrophobic tail, while their double bond central region carries the fluorescent properties. The membrane association of the FM dyes is reversible.

FM 1-43 (N-(3-triethylammoniumpropyl)-4-(4-(dibutylamino) styryl) pyridinium dibromide) is almost non-fluorescent in an aqueous solution but shows an increased fluorescence of more than 40-fold intensity upon binding to liposomes [97].

To characterize the mouse SVs, they were tagged in the FCS measurement using the FM 1-43 dye. The labeling protocol was modified from the previous paper [119]. $10 \mu \mathrm{M}$ FM 1-43 dye was mixed with the same volume of purified SV solution, which was collected directly from size-exclusion chromatography, so that the final concentration of the FM dye was $5 \mu \mathrm{M}$. Incubation was performed for at least $2 \mathrm{~min}$ on ice. Figure 3.12 shows two fluorescence traces taken from two measurements of $10 \mathrm{~s}$ each, with either $5 \mu \mathrm{M}$ FM 1-43 alone or FM-SV-mixture, in which FM dye had a final concentration of $5 \mu \mathrm{M}$ and SV sample was 1:1 diluted by mixing with the FM dye solution. The SVs were clearly labeled with the FM dye.

\subsubsection{Concentration determination of SVs using TP-FCS}

The concentration of the SVs was determined using two photon confocal microscopy at $40 \mathrm{~mW}$ and calculated with fluorescence correlation technique (FCS). The experimental setup was as described previously (page 39, subsection 2.2.8) with a few modifications. Only one APD detector (APD 2) was used to collect either RG or FM 143 fluorescence. Hence, a second dichroic mirror (DC 2) was not included for this measurement, but instead Bandpass filters HQ535/50 as well as HQ645/75 were used to select the emission of either RG or FM dye. 


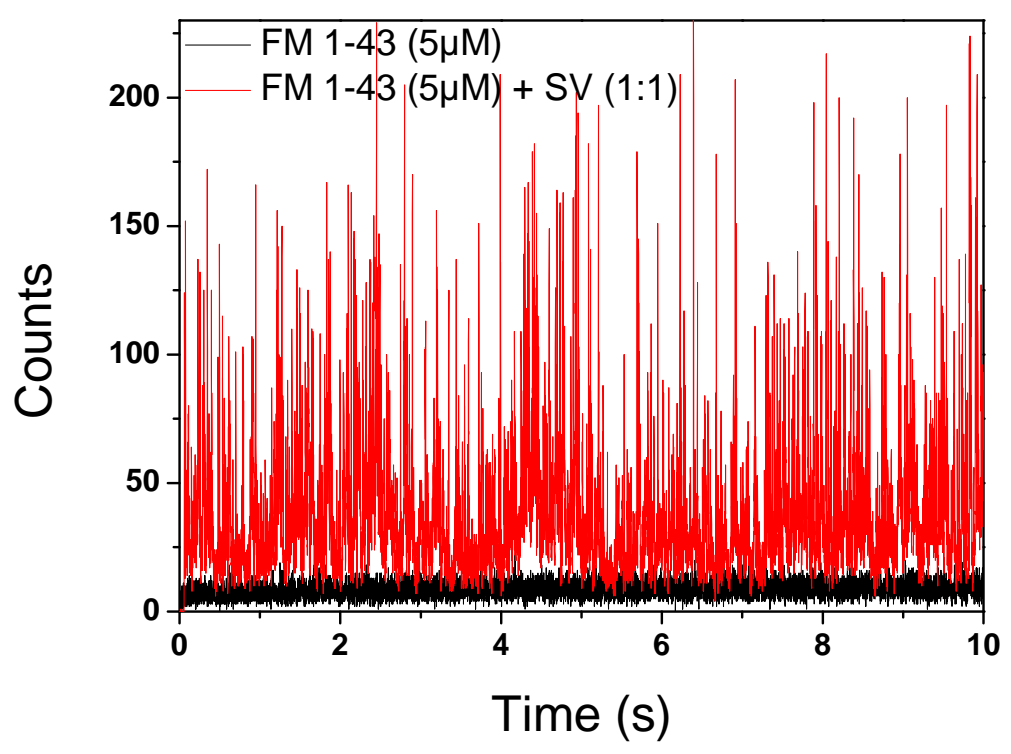

Figure 3.12: SV labeling with FM 1-43 dye. SVs were incubated with the FM 1-43 dye for 2 min on ice with a final concentration of $5 \mu \mathrm{M}$ FM dye (red); a background trace of $5 \mu \mathrm{M}$ FM 1-43 is shown as comparison (black).

The most concentrated SV fraction collected from the size exclusion chromatography (see page 38, subsection 2.2.6 was freshly labeled with FM 1-43 membrane dye (see page 59, subsection 3.2.1). The homogeneity of the SVs was controlled by measuring diffusion time. At least two SV dilutions with a final FM concentration of $5 \mu \mathrm{M}$ were measured (Figure 3.13), and each dilution was measured at least twice with fresh droplets. Every measurement took $6 \times 10 \mathrm{~s}$. The particle number of SVs in the effective volume was calculated with equation 1.6 , by $N_{S V}=1 / G(0)$.

A standard RG fluorescence dye solution with a concentration of $10 \mathrm{nM}$ (page 35 , subsection 2.2.3 was used to calculate the concentration of the SVs. With the particle number $N_{R G}$ of RG the concentration of SVs could be calculated as $c_{S V}=10 \mathrm{nM} \cdot \frac{N_{S V}}{N_{R G}}$. The final concentration was an average of all the dilutions and the error bar was taken to be the standard deviation of all the measurement fragments combined. The data set will be shown in subsection 3.2.4 in combination with the protein concentration. 


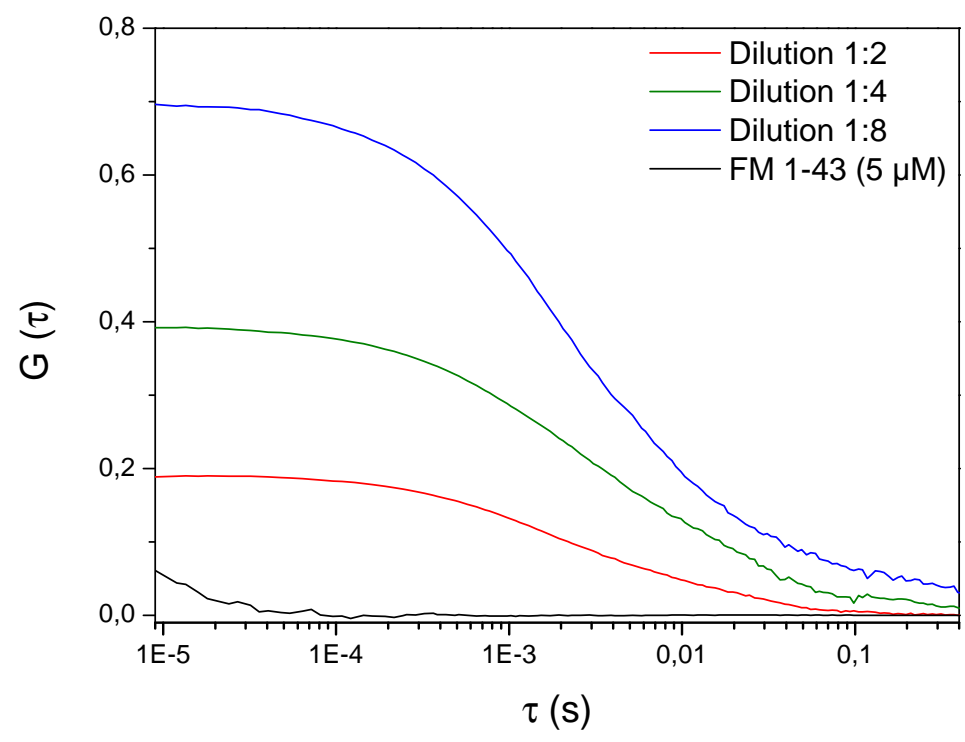

Figure 3.13: FCS measurement of rat SVs in three dilutions compared to FM 1-43 background. All three dilutions were measured in the presence of $5 \mu \mathrm{M}$ FM 1-43 (red, green and blue line). $5 \mu \mathrm{M}$ FM 1-43 is shown as background (black).

\subsubsection{Background correction due to the dark FM fluorescence}

Although the free FM 1-43 dye is almost non-fluorescent [97], its emission at the high concentration used in this experiment was still detectable. To eliminate this "dark" background, a new calculation of the correlation function considering the experimentally observed fluorescence $I^{\exp }(t)$ was developed.

The "dark" emission of $5 \mu$ M FM 1-43 was approximately a constant $f$ independent of time. Assuming that only a small part of the excess FM 1-43 dye was attached to SVs, the experimentally observed fluorescence intensity $I^{\exp }(t)$ could be calculated as the sum of the labeled SV fluorescence $I(t)$ and the FM's near constant emission $f$. The average of the experimentally obtained fluorescence could then be written in the form 
of a sum of the average fluorescence of labeled SVs $\langle I(t)\rangle$ and the FM constant $f$ :

$$
\begin{gathered}
I^{\exp }(t)=I(t)+f \\
\left\langle I^{\exp }(t)\right\rangle=\langle I(t)\rangle+f
\end{gathered}
$$

According to equations 1.1 and 1.2 , the fluorescence correlation function can be written as:

$$
G(\tau)=\frac{\langle(I(t)-\langle I(t)\rangle) \cdot(I(t+\tau)-\langle I(t)\rangle)\rangle}{\langle I(t)\rangle^{2}}
$$

Combining all three preceding equations, the experimentally determined correlation is related to the pure labeled SV signal by the following equation:

$$
\begin{aligned}
G^{\exp }(\tau) & =\frac{\left\langle\left(I^{\exp }(t)-\left\langle I^{\exp }(t)\right\rangle\right) \cdot\left(I^{\exp }(t+\tau)-\left\langle I^{\exp }(t)\right\rangle\right)\right\rangle}{\left\langle I^{\exp }(t)\right\rangle^{2}} \\
& =\frac{\langle((I(t)+f)-(\langle I(t)\rangle+f)) \cdot((I(t+\tau)+f)-(\langle I(t)\rangle+f))\rangle}{(\langle I(t)\rangle+f)^{2}}
\end{aligned}
$$

The numerator of the function results in the same as in equation 3.7 , and $G^{\exp }(\tau)$ can be cast into a new form of $G(\tau)$ multiplied with a constant factor:

$$
G^{\exp }(\tau)=G(\tau) \cdot \frac{\langle I(t)\rangle^{2}}{(\langle I(t)\rangle+f)^{2}}=G(\tau) \cdot \frac{\left(\left\langle I^{\exp }(t)\right\rangle-f\right)^{2}}{\left\langle I^{\exp }(t)\right\rangle^{2}}
$$

Thus, the autocorrelation $G(\tau)$ of labeled SVs can be calculated with the experimentally obtained correlation $G^{\exp }(\tau)$ and a correction factor

$$
G(\tau)=G^{\exp }(\tau) \cdot \frac{\left\langle I^{\exp }(t)\right\rangle^{2}}{\left(\left\langle I^{\exp }(t)\right\rangle-f\right)^{2}}
$$

In the experiment, the average fluorescence intensity $\left\langle I^{\exp }(t)\right\rangle$ is measured in units of photon counts per second. $5 \mu \mathrm{M}$ FM 1-43 was measured separately to obtain the background constant $f$. The calculated correction factor to $G^{\exp }(\tau)$ was determined to be approximately 1.5 .

\subsubsection{Comparing the mass of proteins per vesicle of mouse and rat SVs}

If the particle concentrations of SVs were known, many biological properties could be calculated at the level of a single vesicle. Combining the protein concentration of 
the same SV sample determined by using a modified Lowry-Peterson method (page 35 , subsection 2.2.2.3, the average protein mass per SV could be calculated. Figure 3.14 shows the plotted data of the vesicle concentration versus the protein concentration of the same sample. Besides the measurements for mouse SVs (Figure 3.14, black), two preparations of rat SVs purified by the same method were also measured (Figure 3.14 , red) for a direct comparison with the literature [119]. The mass of proteins per vesicle was calculated as

$$
m^{\text {Pro }}=\frac{c^{\text {Pro }}}{c^{S V} \cdot N_{L}}
$$

in which $c^{\text {Pro }}$ is the protein concentration, $c^{S V}$ is the vesicle concentration, and $N_{L}$ is Avogadro's number.

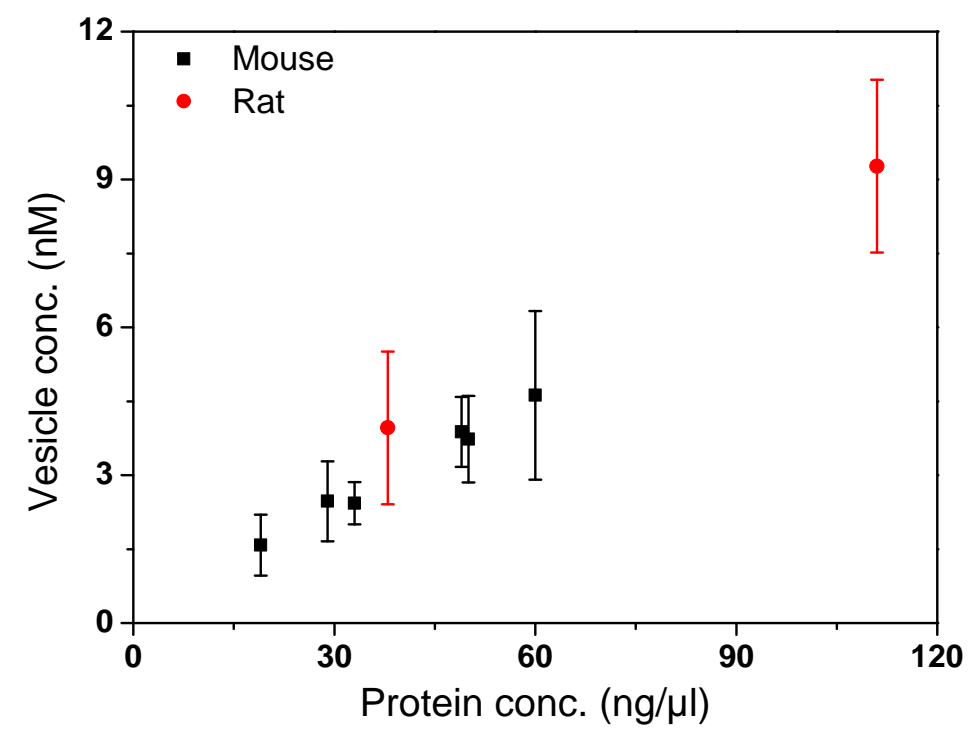

Figure 3.14: SV concentration plotted versus the protein concentration of the same sample. Besides mouse SVs (black), two rat SV samples purified using the same method (red) were plotted as a direct comparison.

The average vesicular protein mass of mouse SVs was calculated as the weighted average of all the single purifications: $m^{\text {Pro }}=(21.4 \pm 2.1) \times 10^{-18} \mathrm{~g} /$ vesicle, corresponding to $12.9 \pm 1.3 \mathrm{MDa}$. The average mass for rat SVs obtained from two purifications was $(18.8 \pm 3.2) \times 10^{-18} \mathrm{~g} /$ vesicle, corresponding to $11.3 \pm 1.9 \mathrm{MDa}$ (Figure 3.15) 
which reflected the previous study measured with scanning transmission electron microscopy (STEM) [119].

Figure 3.15: Calculated protein mass per SV for mouse compared to rat. For one single $\mathrm{SV}$, the average protein mass for mouse was $m^{\text {Pro }}=$ $(21.4 \pm 2.1) \times 10^{-18} \mathrm{~g} /$ vesicle (black), whereas for rat it was $(18.8 \pm 3.2) \times 10^{-18} \mathrm{~g} /$ vesicle $(\mathrm{red})$

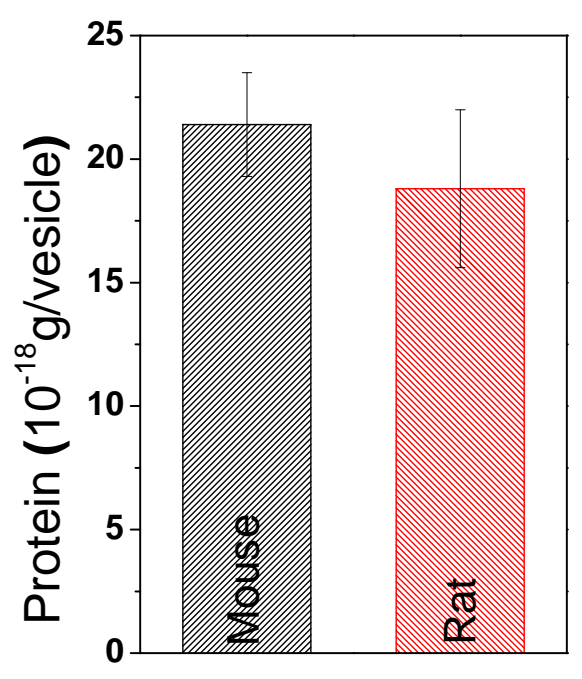

\section{3 $\alpha$-SNAP inhibits SNARE-mediated membrane fusion but not partial SNARE-zippering}

Neuronal SNARE proteins synaptobrevin $2(\mathrm{Sb})$, syntaxin 1A (Sx) and SNAP 25A (SNAP25) assemble from their N-termini toward their C-termini, pull the synaptic vesicle membrane and the presynaptic membrane together and and provide free energy, which is essential to open the fusion pore [63, 79, 91, 116]. The disassembling of the SNAREs requires AAA+ ATPase NSF ( $N$-ethylmaleimide-sensitive factor) [81, 115] and its co-factors SNAPs (soluble NSF attachment proteins), which attach NSF to the SNARE complex, and proceeds via an enzymatic activity of NSF [22].

$\alpha$-SNAP is one of the most abundant isoforms of SNAP proteins [94]. It possesses an $\alpha 1$ region at its $\mathrm{N}$-terminus which is essential for binding to the Q-SNARE complex, so that deletion of the N-terminus completely disrupts the SNARE association [52]. Furthermore, $\alpha$-SNAP consists of a hydrophobic loop at the N-terminus which is responsible for membrane attachment [132] and shows SNARE-independent membrane lipid binding [110]. This membrane attachment also facilitates the disassembly of the 
SNARE complex [132].

Besides its regulatory function during membrane priming and fusion in cooperation with NSF [18, 83, 120, 133], free $\alpha$-SNAP inhibits vesicle fusion and thus the exocytosis in various biological systems [4, 9, 103, 120, 127]. However, the details of this effect are yet unclear.

In a previous study of Y. Park and coworkers (unpublished data), fusion between purified chromaffin granules (CGs) and reconstituted large liposomes in the present of $\alpha$-SNAP was investigated. CGs are one of the different kinds of secretory vesicles isolated from the chromaffin cells of bovine adrenal medullae and are also classified as large dense-core vesicles (LDCVs), since they have an average size of about $200 \mathrm{~nm}$ and consist of an electron-dense core [26]. For their fusion process, CGs contain the same synaptobrevin and synaptotagmin as synaptic vesicles and are used as a model to study SNARE-mediated exocytosis [86]. The large liposomes had an average diameter of about $100 \mathrm{~nm}$ and were reconstituted with either 2:1 complexes (2 Sx:1 SNAP25) [35] or $\Delta \mathrm{N}$ complexes (Sx, SNAP25 and Sb ${ }^{49-96}$; see page 24. Table 2.2). The $\Delta \mathrm{N}$ complex is a stabilized Q-SNARE complex using a small $\mathrm{Sb}^{49-96}$ fragment which can be displaced during assembly with the $\mathrm{Sb}$ of the target membrane and thus promotes rapid membrane fusion in vitro [91]. Besides $\alpha$-SNAP, two N-terminal mutants were also used to determine the functional requirements. The mutants were $\alpha$-SNAP $33-295$ with deleted N-terminus and $\alpha$-SNAP $F 27,28 S$, which has two point mutations on hydrophobic loop between the $\alpha 1$ and $\alpha 2$ helices at the $\mathrm{N}$-terminus.

The fluorescence dequenching spectroscopy and the fluorescence anisotropy data confirmed that $1 \mu \mathrm{M} \alpha$-SNAP clearly inhibited CG fusion by blocking the SNARE assembly. Surprisingly, deletion of the Q-SNARE binding N-terminus $\left(\alpha-\mathrm{SNAP}^{33-295}\right)$ or the membrane binding loop $\left(\alpha-\mathrm{SNAP}{ }^{F 27,28 S}\right)$ both revoked the inhibition, suggesting that the fusion inhibition of $\alpha$-SNAP requires Q-SNARE binding and membrane attachment. Furthermore, the inhibition function of the $\alpha$-SNAP is dependent on membrane curvature, so that an inhibition of fusion was not observed with liposomes with a diameter below $100 \mathrm{~nm}$. In addition, the SNARE assembly during the CG fusion 
was checked using SDS-PAGE in the presence of $\alpha$-SNAP and its mutants. Using the neurotoxin TeNT, which cleaves only the free membrane anchored $\mathrm{Sb}$, the detectable $\mathrm{Sb}$ on the SDS gel indicated the SNARE assembly [51,87]. The SNARE assembly was not fully interrupted by $\alpha$-SNAP and was recovered in the presence of $\alpha$-SNAP ${ }^{F 27,28 S}$ which is in agreement with the results of the fusion experiment.

To answer the question whether the $\alpha$-SNAP blocks the CG fusion but not the SNARE assembly, a set of SNARE-dependent docking experiments using FCS was performed under the same conditions as the CG fusion experiment described above. A diffusion time change [5] of the liposomes upon binding with CGs indicated that $\alpha$-SNAP did not interrupt the CG docking and thus the SNARE zippering. In conclusion, $\alpha$-SNAP inhibits the CG fusion via binding to SNARE complexes with the $\mathrm{N}$-terminus and attaching membranes using the hydrophobic loop, thereby clamping the full SNARE assembly (Figure 3.16).

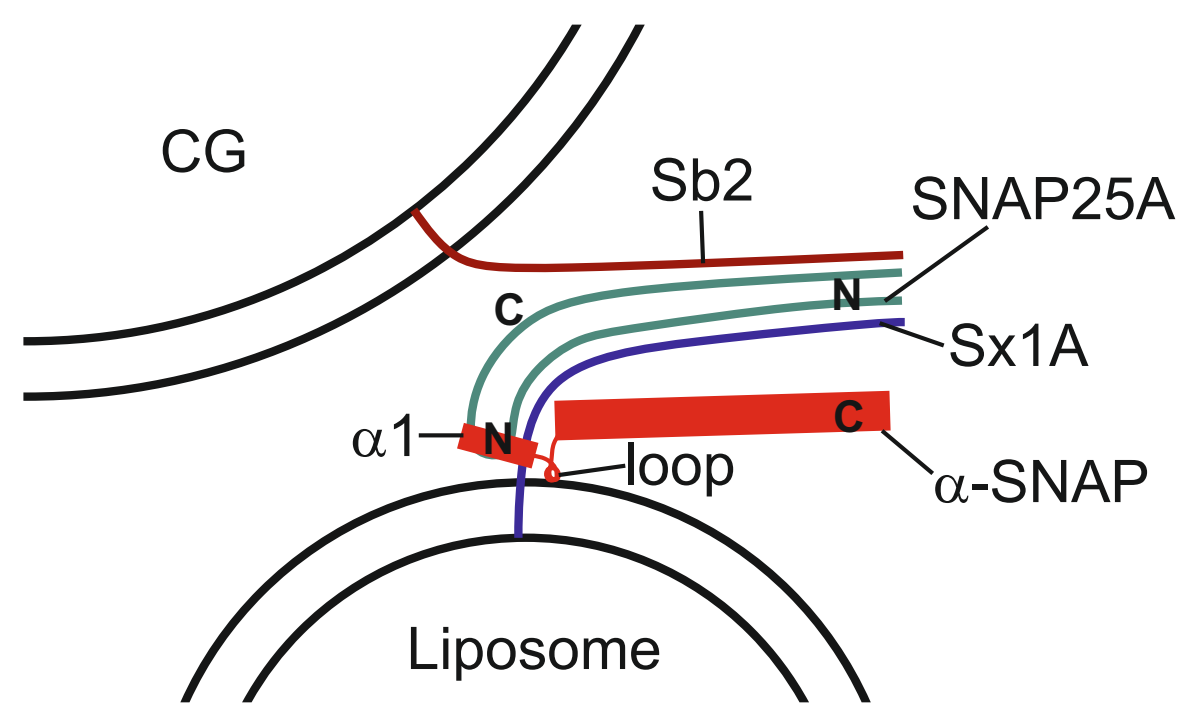

Figure 3.16: Schmatic of CG docking with $100 \mathrm{~nm}$ liposomes in the presence of $\alpha$-SNAP. $\alpha$-SNAP binds to the SNARE complex with its $\mathrm{N}$-terminus and attaches to the membrane via its hydrophobic loop. $\alpha$-SNAP inhibits CG fusion but not the partial SNARE assembly. 


\subsubsection{Determination of CG docking by observing variations in diffu- sion time}

The docking experiments were performed between $\Delta \mathrm{N}$ complex anchored $100 \mathrm{~nm}$ large liposomes (page 37, subsection 2.2.5.2) and purified CGs (page 39, subsection 2.2.7. Here, docking and fusion could not be distinguished, since only the liposome was labeled with both NBD and rhodamine dye, and only the red detection channel was used. Therefore, docking mentioned in this subsection means all the cases where liposome and CG are "gluing" together.

Docking is mediated by SNARE assembly. Using FCS measurements, the changes in liposome diffusion time caused by a size increase upon docking could be determined. The FCS setup was described in a previous section (page 59, subsection 3.2.2) using an HQ645/75 bandpass filter. The average liposome number in the effective volume was about 0.4 , so that single particle docking events could be observed. For the measurement, both $50 \mu \mathrm{g}$ CGs and $10 \mu \mathrm{l}$ liposome were diluted in $1 \mathrm{ml} \mathrm{CG}$ buffer containing K-glutamate and $\mathrm{MgCl}_{2}$ (page 25, Table 2.3). $\alpha$-SNAP was added with a final concentration of $1 \mu \mathrm{M}$. The mixture was incubated for $5 \mathrm{~min}$ at $37^{\circ} \mathrm{C}$. As a negative control, $2 \mu \mathrm{M}$ soluble $\mathrm{Sb}_{1-96}$ was pre-incubated with liposomes at $37^{\circ} \mathrm{C}$ for $30 \mathrm{~min}$, then mixed with CGs, and incubated for another $5 \mathrm{~min}$. $\mathrm{Sb}_{1-96}$ blocks the full SNARE assembly of the $\Delta \mathrm{N}$ complex with the full-length $\mathrm{Sb}$.

Figure 3.17 shows the FCS data of selected docking experiments. Three background diffusion time measurements of each $\Delta \mathrm{N}$ liposomes, protein-free empty liposomes, and empty liposomes in the presence of CG yielded similar diffusion times of about $10 \mathrm{~ms}$ in every case. The negative control experiment with soluble $\mathrm{Sb}_{1-96}$ in the mixture of liposome and CG did not show any changes in diffusion time, as expected (Figure 3.17 first legend session, bottom left). An increased liposome diffusion time of about $50 \mathrm{~ms}$ was observed in both docking experiments in the presence and absence of $\alpha$-SNAP (Figure 3.17 second legend session, top right).

Each docking experiment was measured in $30 \times 10 \mathrm{~s}$ fragments, and the results 


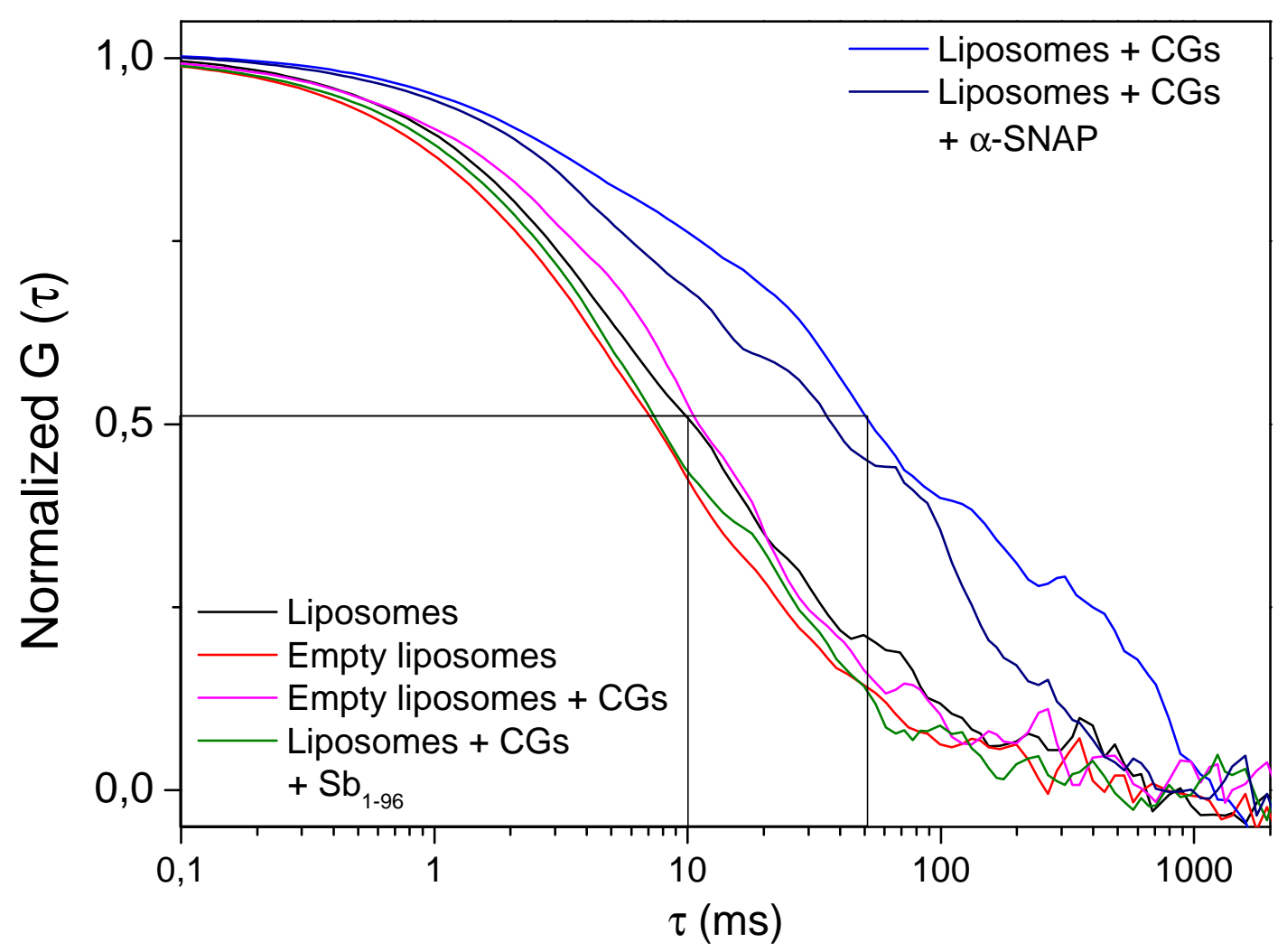

Figure 3.17: Diffusion time determination of the liposome docking experiments with CGs using FCS. Liposomes were reconstituted with $\Delta \mathrm{N}$ complexes. Empty liposomes contained no proteins. The first data set with four FCS curves shows two liposome measurements (black and red), and two negative control measurements, in which either the empty liposomes were mixed with CGs (pink), or the liposomes were mixed with CGs in the presence of soluble $\mathrm{Sb}_{1-96}$ (green). All these no-docking measurements resulted in a diffusion time of about $10 \mathrm{~ms}$. For the positive control measurement, in which $\Delta \mathrm{N}$ liposomes were mixed with CGs (blue), a diffusion time of $50 \mathrm{~ms}$ was measured, indicating that liposomes were docked with CGs. Addition of $\alpha$-SNAP nearly did not change the diffusion time of liposomes (dark blue), suggesting that $\alpha$-SNAP cannot block CG-docking. Except for the two "liposome only" measurements, all the Liposome- CG mixtures were incubated at $37^{\circ} \mathrm{C}$ for $20 \mathrm{~min} . \mathrm{Sb}_{1-96}$ was pre-incubated with liposomes at $37^{\circ} \mathrm{C}$ for $30 \mathrm{~min}$. 
are presented as a histogram of the diffusion time (Figure 3.18). In the cases of the empty liposomes and the negative control experiments, almost all of the 30 measurement fragments showed a diffusion time within $15 \mathrm{~ms}$, which indicated that there was no docking. The docking experiment with $\Delta \mathrm{N}$ liposomes and CGs showed a clearly broader distribution of the diffusion time (Figure 3.18).

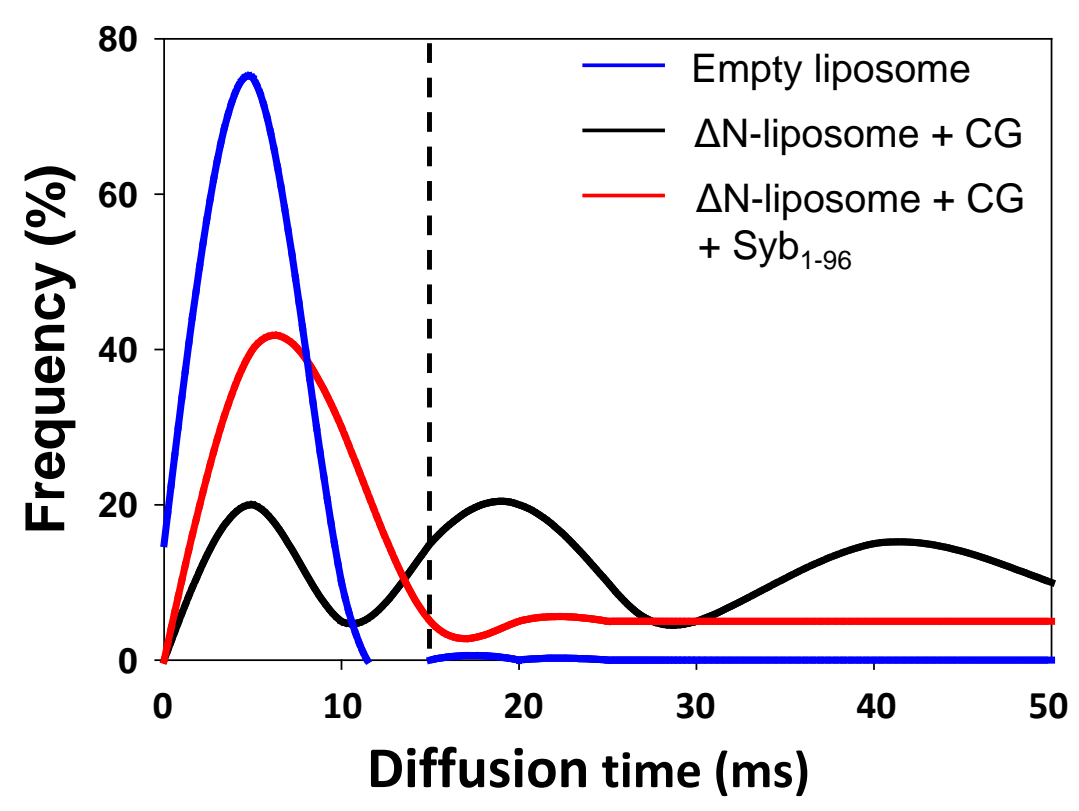

Figure 3.18: Histogram of the docking experiment using liposome and CG. $30 \times 10 \mathrm{~s}$ measurement fragments are plotted as a histogram of diffusion time. Protein-free empty liposomes showed a narrow distribution of the diffusion time within $15 \mathrm{~ms}$ (blue). Repeated fusion experiments with $\Delta \mathrm{N}$ liposomes and CGs showed several occurrences of diffusion times of up tp $50 \mathrm{~ms}$ indicating that a large fraction of the liposomes was docked/fused (black). The negative control experiment performed through pre-incubating with soluble $\mathrm{Sb}_{1-96}$ of the $\Delta \mathrm{N}$ liposomes showed only little docking/fusion to CGs confirming that docking is mediated by SNARE assembly (red). 


\subsection{2 $\alpha$-SNAP does not fully block CG docking}

Following the FCS measurement, $15 \mathrm{~ms}$ were set as the criterion for discriminating docked and free liposomes (Figure 3.18, dash line). The docked liposome (> $15 \mathrm{~ms}$ ) were calculated as percentage of the total of 30 measured events. Each measurement was repeated at least five times using new preparations and the docking rate was obtained as the average and SEM (standard error of mean) of all the measurements (Figure 3.19).

About $65 \%$ of the liposomes were docked (fused) with CGs (Figure 3.19, no addition), and the deletion in the hydrophobic loop $\alpha$-SNAP ${ }^{F 27,28 S}$ did not affect the docking (fusion) which confirmed the previous fusion experiment with CGs (Figure 3.19, F27,28S). The negative control experiment with soluble $\mathrm{Sb}^{1-96}$ showed that docking is mediated by SNARE assembly (Figure 3.19, $\mathrm{Sb}_{1-96}$ ). Surprisingly, $\alpha$-SNAP did not block the docking (about 50\%, Figure 3.19. $\alpha$-SNAP), whereas in the fluorescence dequenching assay $\alpha$-SNAP prevented the CG fusion completely. Docking was reduced to background level using $\mathrm{Sb}_{1-96}$, indicating that docking in the presence of $\alpha$-SNAP is SNARE mediated.

In conclusion, $\alpha$-SNAP is able to inhibit CG fusion via its SNARE binding Nterminus and the membrane attaching loop, but $\alpha$-SNAP does not inhibit partial SNARE assembly and thus liposome docking. 


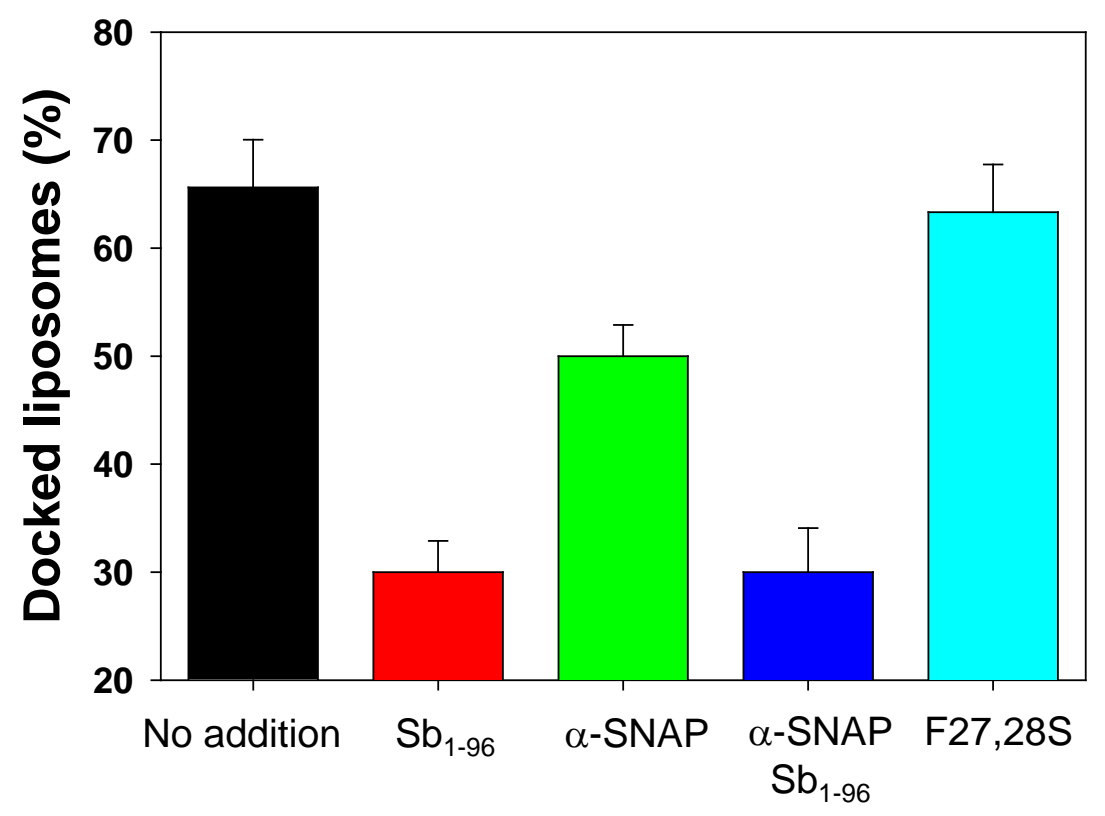

Figure 3.19: $\alpha$-SNAP does not block the SNARE-mediated CG docking. Docking was measured between $\Delta \mathrm{N}$ liposomes and CGs with $5 \mathrm{~min}$ incubation at $37^{\circ} \mathrm{C}$. The docking rate was calculated as percentage of all the 30 measurement fragments. The error is given by SEM of more than five independent measurements. The normal docking/fusion rate was about 65\% without adding $\alpha$-SNAP (black). Docking was found to be reversible using soluble $\mathrm{Sb}_{1-96}$. This indicates that docking is mediated by SNARE assembly (red). Docking was not fully disrupted by $\alpha$-SNAP (green) and was still reduced to the background level using soluble $\mathrm{Sb}_{1-96}$ (blue). Deletion of the hydrophobic loop of $\alpha$-SNAP had no effect on CG docking (light blue), confirming the dequenching fusion experiment. 


\section{Chapter 4}

\section{Discussion}

\subsection{The conflict of cis- and trans-membrane interaction of synaptotagmin-1}

Focusing on the two calcium dependent binding domains $\mathrm{C} 2 \mathrm{~A}$ and $\mathrm{C} 2 \mathrm{~B}$ of synaptotagmin-1, as well as its calcium independent binding site (polybasic lysine stretch) of $\mathrm{C} 2 \mathrm{~B}$, the contribution of each of these independent binding regions to the Syt1 mediated membrane tethering was investigated using a sensitive tethering assay based on FCCS.

In this study, all the three binding sites engage in the tethering process of the membrane anchored Syt1 to the target membrane containing $20 \%$ PS or both $20 \%$ PS and $1 \% \mathrm{PiP} 2$. Less than $50 \%$ tethering caused by the polybasic stretch occurs in the absence of calcium whereas $100 \mu \mathrm{M} \mathrm{CaCl}_{2}$ can almost double the tethering rate with at least one active $\mathrm{C} 2$ domain. This result confirms figures from the previous studies using isolated C2 domains [3, 6, 95]. Membrane anchorage does not affect the trans interaction of the C2 domains (Figure 4.1 a). Similarly, Syt1 also tethers the membrane anchored SNARE complexes. However, the binding is mainly based on the polybasic stretch of $\mathrm{C} 2 \mathrm{~B}$ and does not show a significant dependency on calcium (Figure $4.1 \mathrm{p}$ ). Particularly, binding to the membrane anchored single Sx1A is slightly enhanced by calcium, in agreement 
with previous reports [7, 19, 21, 125].

a

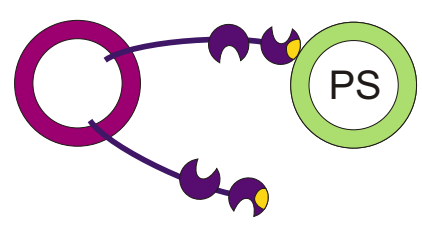

b

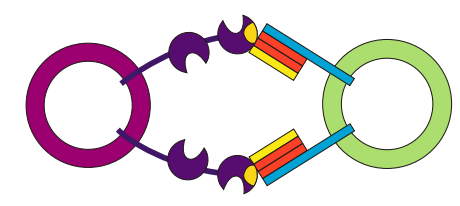

Figure 4.1: Model of the trans-tethering of the membrane anchored Syt1 to the target membrane containing either acidic phospholipids or SNAREs. a. Membrane anchored Syt1 tethers the target liposome in both calcium dependent and independent manners. Target membrane contains either $20 \%$ PS or both $20 \%$ PS and $1 \%$ PiP2. b. Syt1 binds the membrane anchored SNAREs without any calcium enhancement except the binding with the isolated Sx1A.

Surprisingly, 20\% PS in the host membrane of Syt1 completely inhibits the transtethering to the target membrane in all the cases, indicating that Syt1 interacts in cis to its host membrane (Figure 4.2). In contrast to this finding, both soluble C2AB fragment and single $\mathrm{C} 2 \mathrm{~B}$ domain were reported to be able to cluster the vesicles in several studies [3, 111, 141].

It is conceivable that clustering requires at least two independent binding sites. The experimental finding in this study using soluble $\mathrm{C} 2 \mathrm{AB}$ fragments confirms this finding. However, clustering is only observable if $\mathrm{C} 2 \mathrm{AB}$ fragments are saturated on the liposome surface (Figure 4.3), which reflects the case investigated in previous studies. Below the concentration at which binding is saturated, C2AB binds only to one liposome.

There is no direct explanation from the experimental results, why C2AB only clusters liposomes under saturating conditions, since all the liposomes in the solution are similarly occupied. Nevertheless, the space and orientation requirements for placing 
a
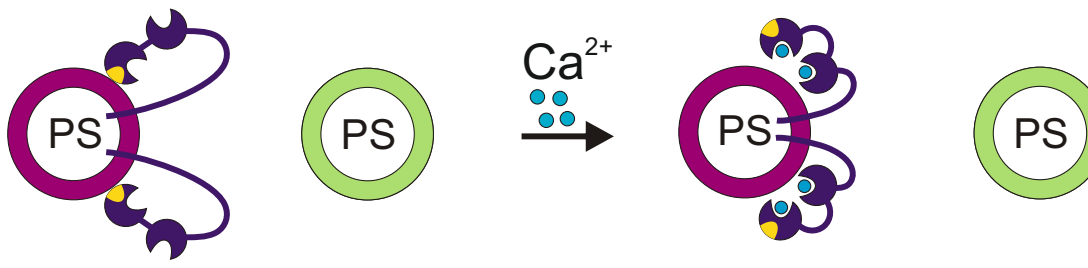

b
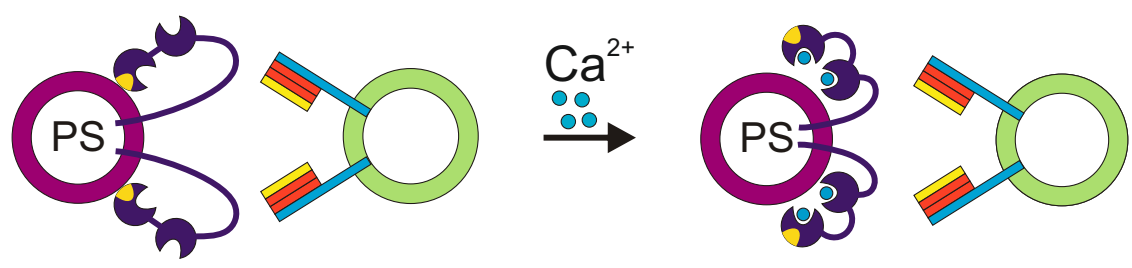

Figure 4.2: All trans-tethering is inhibited if the host liposome of Syt1 contains $20 \%$ PS. Target liposome contains either acidic phospholipids (a) or SNAREs (b). No tethering is observable even in the presence of $100 \mu \mathrm{M} \mathrm{CaCl}_{2}$.

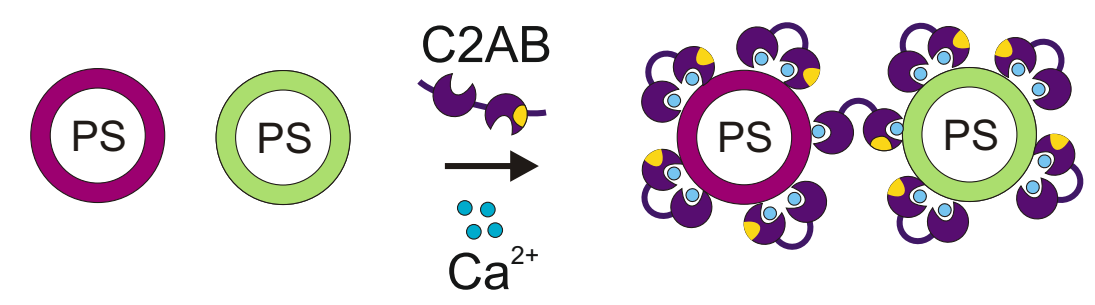

Figure 4.3: $\mathrm{C} 2 \mathrm{AB}$ fragment is able to cluster the liposome under a saturating concentration on the liposome surface. Clustering is reversible by adding 2 mM EGTA. 
both $\mathrm{C} 2$ domains of one $\mathrm{C} 2 \mathrm{AB}$ fragment on the membrane surface may still leave a smaller area which is barely sufficient to bind one C2 domain, and furthermore to cross-link a second liposome. Alternatively, cross-linking is only observable when the liposome surface is fully covered by $\mathrm{C} 2 \mathrm{AB}$ fragments, so that a maximum number of $\mathrm{C} 2 \mathrm{AB}$ bridges are achieved to stabilize the membrane tethering. With these findings, many seemingly contradictory reports in the literature could be harmonized [3,60].

In conclusion, the cis-binding model confirms the previous studies, in which membrane anchored Syt1 reduces the fusion efficiency in the in vitro experiments [71.111], and exhibits a declaration for this observation, that the $\mathrm{C} 2$ domains of Syt 1 bind in cis orientation to its host membrane containing acidic phospholipids and become inactive for cross-linking the plasma membrane. Some of the conflicting reports about Syt1 in artificial systems may be thus explained [67, 71, 111].

Along with this study, similar results have been reported [65] based on a related experimental design [23], which are largely in agreement with this study. Complementing data has also shown that SNAREs and Syt1 involved in membrane fusion is only calcium stimulated, if excess PS is present in the target membrane of Syt1 [70]; which is in accord with the tethering experiments using low percentage PS (5\%) in the host liposome of Syt1.

Most importantly, the results have emphasized the conflict of cis- vs. transmembrane interaction of Syt1. Cis binding of the membrane anchored Syt1 is still observable even if the PS composition is lowered to $12 \%$ in the host membrane, which is comparable to the natural synaptic vesicles containing more than $15 \%$ anionic phospholipids, suggesting that cis-interaction may occur under physiological conditions. This has been confirmed by several preliminary data using SVs and CGs (unpublished data and [58]). It is conceivable that in a docked intermediate, the vesicle membrane and the plasma membrane get close enough to compensate for the cis-binding, and one of the $\mathrm{C} 2$ domains can trans bind the plasma membrane - probably $\mathrm{C} 2 \mathrm{~B}$ due to its special ability to PiP2 [6, 73, 104, 134, 141] - and thus mediate the cross-linking [67, 141]. Alternatively, cis-binding may be prevented by some other factors, such as molecular 
crowding or charge screening. The latter has recently been observed [89], i.e. that $5 \mathrm{mM}$ polyvalent anions ATP can shield the cis-membrane interaction, since the calcium dependent membrane affinity of Syt1 is similar to the calcium affinity of ATP, and ATP competes with the lipid membrane for binding Syt1 in a calcium dependent manner. However ATP can only prevent the membrane binding of Syt1 if the PS concentration is not higher than $15 \%$ and no PiP2 is present. Again, $15 \%$ PS is comparable to the natural lipid composition in SVs and CGs. On the other hand, 1\% PiP2 enhances the calcium binding between Syt1 and the membrane by more than a factor of four, possibly due to the direct binding of the polybasic stretch of the C2B domain to PiP2 [89]. In conclusion, Syt1 binds the vesicular membrane in cis in the absence of ATP and becomes inactive for cross-linking the plasma membrane, whereas ATP interrupts the cis-binding of Syt1 and activates the C2 domains to bind the plasma membrane containing PiP2 [89]. This finding is important, because the shielding model of ATP may balance the cis- and trans-membrane interaction of Syt1 and thus modulate the membrane fusion. Cross-linking could be thought of as an "intermediate" of both cis-and trans-binding, so that the $\mathrm{C} 2 \mathrm{~B}$ domain interacts with PiP2 in the plasma membrane and C2A backbinds to the vesicular membrane [141]. If this is the case, it is still not clear whether the cross-linking proceeds in a single step or in a sequence of steps.

Moreover, the trans-binding of Syt1 already tethers the vesicle to the plasma membrane, since Syt1 is anchored in the vesicular membrane by the transmembrane domain, raising the question whether a calcium dependent cross-linking is still required for its function in membrane fusion.

The advantage of a cross-linking model is to lower the distance between the membranes and promote the SNARE assembly [141]. Finally, it cannot be ruled out that calcium dependent cis-binding is sufficient for triggering the membrane fusion, in such fashion as to induce change in the vesicular membrane curvature. 


\section{2 $\alpha$-SNAP inhibits SNARE-mediated CG fusion, but does not fully block CG docking}

Neuronal exocytosis is mediated by SNAREs assembly, termed "zippering" of the vesicular protein synaptobrevin and the Q-SNARE proteins syntaxin and SNAP-25 located in the target membrane [63]. The zippering proceeds from the $\mathrm{N}$-terminus to the C-terminus [91]. $\alpha$-SNAP has been reported to inhibit vesicle fusion [4,9, 103, 120, 127]. The preliminary fusion experiments between CGs and reconstituted liposome performed in the neurobiology department have shown that $\alpha$-SNAP completely prevents CG fusion and that the inhibition requires binding of the $\alpha 1$ region of the $\mathrm{N}$-terminus to the C-terminus of the Q-SNARE complex near the membrane and the membrane attachment of the hydrophobic loop of the N-terminus (unpublished data). As an extension of these results, docking between the purified CGs and the $100 \mathrm{~nm}$ liposome was investigated in the presence of $\alpha$-SNAP or its N-terminus mutants $\left(\alpha-\mathrm{SNAP}^{d e l}\right.$ and $\alpha$ SNAP ${ }^{F 27,28 S}$ ). Liposomes contained stabilized Q-SNARE complex. Docking was monitored via diffusion time changing using FCS. Surprisingly, docking was still observable under the same conditions, by which fusion is prevented. The negative control using soluble $\mathrm{Sb}^{1-96}$ restored the docking, indicating that docking is SNARE dependent. In conclusion, $\alpha$-SNAP blocks the CG fusion at the C-terminal side of the SNARE complex (unpublished data of the neurobiology department), but does not prevent the partial SNARE assembly starting from the SNARE N-terminal end, and preserves the vesicle in a docked intermediate.

This unexpected result has never been observed before and sheds light to the regulatory role of the $\alpha$-SNAP in membrane fusion. However it is necessary to question whether a partial assembly of SNARE proteins at the C-terminal side is conceivable. The ternary SNARE complex structures has highly conserved layers, in which the side chains of the amino acid interact in the "tube" of the four SNARE motives [36]. Furthermore, the X-ray study suggested that the SNARE helical bundle is extended from the SNARE motives to the linker region by the further side chain interaction and the 
linker region directly contributes to membrane fusion [112]. It has also been reported, that the transition region between the SNARE motives and the linker region plays an important role in membrane fusion [106, 109, 126]. Due to these, a single deletion mutant of synaptobrevin was introduced at position 84 ( $\mathrm{Sb} \Delta 84)$, which consists the +8 layer of the SNARE helical bundle and is directly followed by the linker region [55]. $\mathrm{Sb} \Delta 84$ bearing liposomes cannot fuse if the liposome contains stabilized Q-SNARE complex ( $\Delta \mathrm{N}$ complex) as long as at least one of the liposome populations is comprised of large liposomes (radius from 40 to $100 \mathrm{~nm}$ ), since the +8 layer is disrupted by deletion. However, the FCCS results combined with FRET lifetime analysis [23] have shown that $\mathrm{Sb} \Delta 84$ still docks the large liposome, indicating that partial SNARE assembly still proceeds under these conditions [55]. Although this result cannot be directly compared with the $\alpha$-SNAP mediated partial SNARE assembly, it suggests a scenario in which SNARE assembly can be halted at the C-terminus end, if the layer structure of the SNARE complex is disrupted. Thereby, it is conceivable that the interaction between the $\alpha 1$ region of $\alpha$-SNAP and the SNARE's C-terminal side near the membrane may be strong enough to deform one of the layers and dock the liposomes by partial SNARE assembly. Finally, it has to be pointed out, that the increased membrane curvature of the small liposome (diameter $45 \mathrm{~nm}$ ) overcomes the inhibition of $\alpha$-SNAP and promotes the membrane fusion (unpublished data from Dr. Yongsoo Park). 


\subsection{Quantitative characterization of synaptic vesicles}

Using a sensitive vesicle counting assay based on TP-FCS, freshly isolated homogenous mouse synaptic vesicles with low density were characterized in terms of the particle number. The membrane labeling dye FM 1-43 was used to visualize the vesicles. Cooperating with the neurobiology department, the total protein mass of the same vesicle sample was determined using a modified Lowry-Peterson method. Combining both results, an averaged protein mass per single synaptic vesicle was obtained.

The result is very well related to the previous report of rat synaptic vesicle [119], indicating that the two different species have a similar property of the synaptic vesicles despite of their morphological differences in terms of body - and brain size. In particular, mouse synaptic vesicles have a larger protein mass than those of obtained from rats $(\sim 20 \%)$. With the known vesicle number as a starting point, many biological parameters can be identified at a single vesicle level, for instance protein composition and lipid composition. 


\section{Chapter 5}

\section{Conclusions}

Neurotransmission requires rapid fusion of synaptic vesicles with the presynaptic membrane. This fusion is triggered by calcium influx and promoted by SNARE assembly. The vesicular protein synaptotagmin- 1 acts as a calcium sensor and its transtethering to the plasma membrane is an essential step in the membrane fusion process. In this study, several details in membrane fusion have been investigated and discussed.

First, the molecular requirements of the trans- and cis-membrane interaction of synaptotagmin- 1 have been analyzed. All of the three binding sites of synaptotagmin-1 (the $\mathrm{C} 2 \mathrm{~A}$ domain, the $\mathrm{C} 2 \mathrm{~B}$ domain and the polylysine patch) are involved in both of these processes. Cis-binding to the host membrane containing 20\% phosphatidylserine seems to be a stable state and can be overcome by neither trans-binding between synaptotagmin- 1 and the target membrane containing acidic phospholipids, nor by the interaction between synaptotagmin-1 and SNAREs inserted into the acceptor membrane. Similarly, with $12 \%$ phosphatidylserine in the host membrane-a situation comparable to that in wild-type vesicles [119]-cis-binding is found nearly exclusively. With 5\% phosphatidylserine, however, the presence of either phosphatidylinositol-4,5bisphosphate in the target membrane or calcium in the solution can invoke conversion to trans-binding with great efficiency.

Second, liposome cross-linking can be mediated by soluble C2AB fragments, when 
all the binding sites for $\mathrm{C} 2 \mathrm{AB}$ are saturated on the liposome surface. To rationalize this result, it is assumed that binding with only the C2A- or the C2B domain might occur under these conditions. The other-unbound-domain of the same synaptotagmin molecule will eventually lead to cross-linking, if it attaches to a different liposome. This finding confirms the observation of cis-binding and explains some of the conflicts in the existing literature. Apparently, a subtle balance between cis- and trans-binding of synaptotagmin-1 may play an important role in the regulation of neuronal membrane fusion.

Moreover, first data for the characterization of mouse synaptic vesicles, in terms of average protein mass per vesicle, was measured in this study. The results obtained for mouse synaptic vesicles are similar to those of rat synaptic vesicles and offer a single vesicle basis for further investigating mouse synaptic vesicles as well as membrane fusion in a quantitative fashion.

Finally, details of the inhibiting effect of $\alpha$-SNAP on the fusion of chromaffin granules with larger liposomes were also investigated in the present study. The experimental findings suggest that $\alpha$-SNAP inhibits fusion of chromaffin granules and stops the SNARE assembly at the C-terminal site, so that partial SNARE zippering can still mediate docking of chromaffin granules. 


\section{Bibliography}

[1] Ahmed, S. Isolation and Characterization of Synaptic Vesicles from Mouse Brain. PhD thesis, Georg-August-University Göttingen, 2010.

[2] Angleson, J. K., Cochilla, A. J., Kilic, G., Nussinovitch, I., And BetZ, W. J. Regulation of dense core release from neuroendocrine cells revealed by imaging single exocytic events. Nat Neurosci 2, 5 (May 1999), 440-446.

[3] Araç, D., Chen, X., Khant, H. A., Ubach, J., LudtKe, S. J., KikKawa, M., Johnson, A. E., Chiu, W., SÜdhof, T. C., And Rizo, J. Close membrane-membrane proximity induced by $\mathrm{Ca}^{2+}$-dependent multivalent binding of synaptotagmin-1 to phospholipids. Nat Struct Mol Biol 13, 3 (Mar 2006), 209-217.

[4] BABCOCK, M., MACleod, G. T., Leither, J., AND PAllancK, L. Genetic analysis of soluble N-ethylmaleimide-sensitive factor attachment protein function in Drosophila reveals positive and negative secretory roles. J Neurosci 24, 16 (Apr 2004), 3964-3973.

[5] BACIA, K., AND SCHWILlE, P. Fluorescence correlation spectroscopy. Methods Mol Biol 398 (2007), 73-84.

[6] Bai, J., Tucker, W. C., And Chapman, E. R. PIP2 increases the speed of response of synaptotagmin and steers its membrane-penetration activity toward the plasma membrane. Nat Struct Mol Biol 11, 1 (Jan 2004), 36-44. 
[7] Bai, J., WANG, C.-T., Richards, D. A., JaCKson, M. B., AND Chapman, E. R. Fusion pore dynamics are regulated by synaptotagmin*t-SNARE interactions. Neuron 41, 6 (Mar 2004), 929-942.

[8] Barnard, R. J., Morgan, A., And Burgoyne, R. D. Stimulation of NSF ATPase activity by $\alpha$-SNAP is required for SNARE complex disassembly and exocytosis. J Cell Biol 139, 4 (Nov 1997), 875-883.

[9] Barszczewski, M., Chua, J. J., Stein, A., Winter, U., Heintzmann, R., ZILly, F. E., FASSHAUER, D., LANG, T., AND JAHN, R. A novel site of action for $\alpha$-SNAP in the SNARE conformational cycle controlling membrane fusion. Mol Biol Cell 19, 3 (Mar 2008), 776-784.

[10] Berland, K. M., So, P. T., And Gratton, E. Two-photon fluorescence correlation spectroscopy: method and application to the intracellular environment. Biophys J 68, 2 (Feb 1995), 694-701.

[11] Bernard, V. Molecular Fluorescence: Principles and Applications. Wiley-VCH, 2001.

[12] BetZ, W. J., MAO, F., AND BEwICK, G. S. Activity-dependent fluorescent staining and destaining of living vertebrate motor nerve terminals. J Neurosci 12, 2 (Feb 1992), 363-375.

[13] Bhalla, A., Chicka, M. C., Tucker, W. C., And Chapman, E. R. Ca ${ }^{2+}$ synaptotagmin directly regulates t-SNARE function during reconstituted membrane fusion. Nat Struct Mol Biol 13, 4 (Apr 2006), 323-330.

[14] Blom, H., Chmyrov, A., Hassler, K., Davis, L., And Widengren, J. Triplet-State Investigations of Fluorescent Dyes at Dielectric Interfaces Using Total Internal Reflection Fluorescence Correlation Spectroscopy. J Phys Chem A 113, 19 (2009), 5554-5566. 
[15] BRADFORD, M. M. A rapid and sensitive method for the quantitation of microgram quantities of protein utilizing the principle of protein-dye binding. Anal Biochem 72 (May 1976), 248-254.

[16] Brumback, A. C., Lieber, J. L., Angleson, J. K., And Betz, W. J. Using FM1-43 to study neuropeptide granule dynamics and exocytosis. Methods 33, 4 (Aug 2004), 287-294.

[17] BRUNGER, A. T. Structure of proteins involved in synaptic vesicle fusion in neurons. Annu Rev Biophys Biomol Struct 30 (2001), 157-171.

[18] Burgalossi, A., Jung, S., Meyer, G., JOCKusch, W. J., Jahn, O., TAschenBerger, H., O'CONNOR, V. M., Nishiki, T.-I., TAKAHASHI, M., Brose, N., AND RHEE, J.-S. SNARE protein recycling by $\alpha$ SNAP and $\beta$ SNAP supports synaptic vesicle priming. Neuron 68, 3 (Nov 2010), 473-487.

[19] Chapman, E. R. How does synaptotagmin trigger neurotransmitter release? Annu Rev Biochem 77 (2008), 615-641.

[20] ChEN, Y. A., AND SCHELlER, R. H. SNARE-mediated membrane fusion. Nat Rev Mol Cell Biol 2, 2 (Feb 2001), 98-106.

[21] Choi, U. B., Strop, P., Vrljic, M., Chu, S., Brunger, A. T., AND Weninger, K. R. Single-molecule FRET-derived model of the synaptotagmin 1-SNARE fusion complex. Nat Struct Mol Biol 17, 3 (Mar 2010), 318-324.

[22] Clary, D. O., GRIfF, I. C., AND Rothman, J. E. SNAPs, a family of NSF attachment proteins involved in intracellular membrane fusion in animals and yeast. Cell 61, 4 (May 1990), 709-721.

[23] Cypionka, A., Stein, A., Hernandez, J. M., Hippchen, H., Jahn, R., And WALLA, P. J. Discrimination between docking and fusion of liposomes reconstituted with neuronal SNARE-proteins using FCS. Proc Natl Acad Sci U S A 106, 44 (Nov 2009), 18575-18580. 
[24] DAuty, E., AND VERKMAN, A. S. Actin cytoskeleton as the principal determinant of size-dependent DNA mobility in cytoplasm: a new barrier for non-viral gene delivery. J Biol Chem 280, 9 (Mar 2005), 7823-7828.

[25] DAVIS, L. M., AND SHEN, G. Accounting for triplet and saturation effects in FCS measurements. Curr Pharm Biotechnol 7, 4 (Aug 2006), 287-301.

[26] De Camilli, P., AND JAhn, R. Pathways to regulated exocytosis in neurons. Annu Rev Physiol 52 (1990), 625-645.

[27] Denk, W., Strickler, J. H., And WebB, W. W. Two-photon laser scanning fluorescence microscopy. Science 248, 4951 (Apr 1990), 73-76.

[28] EDELHOCH, H. Spectroscopic determination of tryptophan and tyrosine in proteins. Biochemistry 6, 7 (Jul 1967), 1948-1954.

[29] EhrenberG, M., AND RigleR, R. Rotational brownian motion and fluorescence intensify fluctuations. Chemical Physics 4, 3 (1974), 390-401.

[30] EINSTEIN, A. Über die von der molekularkinetischen Theorie der Wärme geforderte Bewegung von in ruhenden Flüssigkeiten suspendierten Teilchen. Ann Phys 17 (1905), 549-560.

[31] Elson, E., AND MAGDE, D. Fluorescence correlation spectroscopy I: Conceptual basis and theory. Biopolymers 13 (1974), 1-27.

[32] EXPASY - $\quad$ BioINFORMATICS RESOURCE PORTAL. http:/ / web.expasy.org/protparam/. Accessed on September 25, 2012.

[33] Fasshauer, D., Antonin, W., Margittai, M., Pabst, S., and Jahn, R. Mixed and non-cognate SNARE complexes. Characterization of assembly and biophysical properties. J Biol Chem 274, 22 (May 1999), 15440-15446.

[34] Fasshauer, D., AND Margittai, M. A transient N-terminal interaction of SNAP-25 and syntaxin nucleates SNARE assembly. J Biol Chem 279, 9 (Feb 2004), 7613-7621. 
[35] Fasshauer, D., Otto, H., Eliason, W. K., JAhN, R., AND Brünger, A. T. Structural changes are associated with soluble N-ethylmaleimide-sensitive fusion protein attachment protein receptor complex formation. J Biol Chem 272, 44 (Oct 1997), 28036-28041.

[36] Fasshauer, D., Sutton, R. B., Brunger, A. T., And Jahn, R. Conserved structural features of the synaptic fusion complex: SNARE proteins reclassified as Q- and R-SNAREs. Proc Natl Acad Sci U S A 95, 26 (Dec 1998), 15781-15786.

[37] Fernandez, I., Araç, D., Ubach, J., Gerber, S. H., Shin, O., GaO, Y., AnDERSON, R. G., SÜDHOF, T. C., AND RIZO, J. Three-dimensional structure of the synaptotagmin $1 \mathrm{C} 2 \mathrm{~B}-$ domain: synaptotagmin 1 as a phospholipid binding machine. Neuron 32, 6 (Dec 2001), 1057-1069.

[38] Fiebig, K. M., Rice, L. M., POllock, E., And Brunger, A. T. Folding intermediates of SNARE complex assembly. Nat Struct Biol 6, 2 (Feb 1999), 117-123.

[39] Fleming, K. G., Hohl, T. M., YU, R. C., MÜller, S. A., Wolpensinger, B., Engel, A., Engelhardt, H., Brünger, A. T., SÖllner, T. H., ANd HaNSON, P. I. A revised model for the oligomeric state of the N-ethylmaleimidesensitive fusion protein, NSF. J Biol Chem 273, 25 (Jun 1998), 15675-15681.

[40] Furst, J., Sutton, R. B., Chen, J., Brunger, A. T., And Grigorieff, N. Electron cryomicroscopy structure of N-ethyl maleimide sensitive factor at $11 \AA$ resolution. EMBO J 22, 17 (Sep 2003), 4365-4374.

[41] FÖRSTER, T. Zwischenmolekulare Energiewanderung und Fluoreszenz. Ann Phys 6 (1948), 55-75.

[42] Gaffaney, J. D., Dunning, F. M., Wang, Z., Hui, E., And Chapman, E. R. Synaptotagmin $\mathrm{C} 2 \mathrm{~B}$ domain regulates $\mathrm{Ca}^{2+}$-triggered fusion in vitro: critical residues revealed by scanning alanine mutagenesis. J Biol Chem 283, 46 (Nov 2008), 31763-31775. 
[43] Gill, S. C., AND von Hippel, P. H. Calculation of protein extinction coefficients from amino acid sequence data. Anal Biochem 182, 2 (Nov 1989), 319-326.

[44] Goeppert-Mayer, M. Über Elementarakte mit zwei Quantensprüngen. Annalen der Physik 9 (1931), 273-294.

[45] Granseth, B., Odermatt, B., Royle, S. J., and Lagnado, L. Clathrinmediated endocytosis is the dominant mechanism of vesicle retrieval at hippocampal synapses. Neuron 51, 6 (Sep 2006), 773-786.

[46] Grünwald, D., Cardoso, M. C., Leonhardt, H., and Buschmann, V. Diffusion and binding properties investigated by Fluorescence Correlation Spectroscopy (FCS). Curr Pharm Biotechnol 6, 5 (Oct 2005), 381-386.

[47] GösCH, M., AND RIGLER, R. Fluorescence correlation spectroscopy of molecular motions and kinetics. Adv Drug Deliv Rev 57, 1 (Jan 2005), 169-190.

[48] Hanson, P. I., Heuser, J. E., AND Jahn, R. Neurotransmitter release - four years of SNARE complexes. Curr Opin Neurobiol 7, 3 (Jun 1997), 310-315.

[49] Hanson, P. I., Roth, R., Morisaki, H., Jahn, R., And Heuser, J. E. Structure and conformational changes in NSF and its membrane receptor complexes visualized by quick-freeze/deep-etch electron microscopy. Cell 90, 3 (Aug 1997), 523-535.

[50] HAustein, E., AND SCHWille, P. Fluorescence correlation spectroscopy: novel variations of an established technique. Annu Rev Biophys Biomol Struct 36 (2007), 151-169.

[51] Hayashi, T., McMahon, H., Yamasaki, S., Binz, T., Hata, Y., SÜdhof, T. C., AND NiemanN, H. Synaptic vesicle membrane fusion complex: action of clostridial neurotoxins on assembly. EMBO J 13, 21 (Nov 1994), 5051-5061. 
[52] Hayashi, T., Yamasaki, S., NauenburG, S., Binz, T., AND NiemanN, H. Disassembly of the reconstituted synaptic vesicle membrane fusion complex in vitro. EMBO J 14, 10 (May 1995), 2317-2325.

[53] Hell, J. W., Maycox, P. R., StAdleR, H., AND JAHN, R. Uptake of GABA by rat brain synaptic vesicles isolated by a new procedure. EMBO J 7, 10 (Oct 1988), 3023-3029.

[54] Henkel, A. W., LÜBKe, J., AND BetZ, W. J. FM1-43 dye ultrastructural localization in and release from frog motor nerve terminals. Proc Natl Acad Sci U S A 93, 5 (Mar 1996), 1918-1923.

[55] Hernandez, J. M., Stein, A., Behrmann, E., Riedel, D., CypionkA, A., Farsi, Z., Walla, P. J., RAunser, S., And JAhn, R. Membrane fusion intermediates via directional and full assembly of the SNARE complex. Science 336, 6088 (Jun 2012), 1581-1584.

[56] Herrick, D. Z., Sterbling, S., Rasch, K. A., Hinderliter, A., And CAfiso, D. S. Position of synaptotagmin I at the membrane interface: cooperative interactions of tandem C2 domains. Biochemistry 45, 32 (Aug 2006), 9668-9674.

[57] Hohl, T. M., Parlati, F., Wimmer, C., Rothman, J. E., SÖllner, T. H., AND ENGELHARDT, H. Arrangement of subunits in $20 \mathrm{~S}$ particles consisting of NSF, SNAPs, and SNARE complexes. Mol Cell 2, 5 (Nov 1998), 539-548.

[58] Holt, M., Riedel, D., Stein, A., Schuette, C., And Jahn, R. Synaptic vesicles are constitutively active fusion machines that function independently of $\mathrm{Ca}^{2+}$. Curr Biol 18, 10 (May 2008), 715-722.

[59] HUi, E., BAI, J., AND CHAPMAN, E. R. Ca ${ }^{2+}$-triggered simultaneous membrane penetration of the tandem C2-domains of synaptotagmin I. Biophys J 91, 5 (Sep 2006), 1767-1777. 
[60] Hui, E., Gaffaney, J. D., Wang, Z., Johnson, C. P., Evans, C. S., And CHAPMAN, E. R. Mechanism and function of synaptotagmin-mediated membrane apposition. Nat Struct Mol Biol 18, 7 (Jul 2011), 813-821.

[61] Hui, E., Johnson, C. P., YaO, J., Dunning, F. M., And Chapman, E. R. Synaptotagmin-mediated bending of the target membrane is a critical step in $\mathrm{Ca}^{2+}$-regulated fusion. Cell 138, 4 (Aug 2009), 709-721.

[62] Jahn, R., LANG, T., AND Südhof, T. C. Membrane fusion. Cell 112, 4 (Feb 2003), 519-533.

[63] JAHN, R., AND SCHELlER, R. H. SNAREs-engines for membrane fusion. Nat Rev Mol Cell Biol 7, 9 (Sep 2006), 631-643.

[64] Jahn, R., Schiebler, W., AND Greengard, P. A quantitative dotimmunobinding assay for proteins using nitrocellulose membrane filters. Proc Natl Acad Sci U S A 81, 6 (Mar 1984), 1684-1687.

[65] Kim, J.-Y., ChOI, B.-K., CHOI, M.-G., KIM, S.-A., LAI, Y., SHIN, Y.-K., AND LEE, N. K. Solution single-vesicle assay reveals PIP2-mediated sequential actions of synaptotagmin-1 on SNAREs. EMBO J 31, 9 (May 2012), 2144-2155.

[66] Kuner, T., Li, Y., Gee, K. R., Bonewald, L. F., And Augustine, G. J. Photolysis of a caged peptide reveals rapid action of $\mathrm{N}$-ethylmaleimide sensitive factor before neurotransmitter release. Proc Natl Acad Sci U S A 105, 1 (Jan 2008), 347352.

[67] Kyoung, M., Srivastava, A., Zhang, Y., Diao, J., Vrljic, M., Grob, P., Nogales, E., CHU, S., AND BRunger, A. T. In vitro system capable of differentiating fast $\mathrm{Ca}^{2+}$-triggered content mixing from lipid exchange for mechanistic studies of neurotransmitter release. Proc Natl Acad Sci U S A 108, 29 (Jul 2011), E304-E313.

[68] LAEMMLI, U. K. Cleavage of structural proteins during the assembly of the head of bacteriophage T4. Nature 227, 5259 (Aug 1970), 680-685. 
[69] Lai, A. L., Huang, H., Herrick, D. Z., Epp, N., AND CAfiso, D. S. Synaptotagmin 1 and SNAREs form a complex that is structurally heterogeneous. J Mol Biol 405, 3 (Jan 2011), 696-706.

[70] LAI, Y., AND SHIN, Y.-K. The importance of an asymmetric distribution of acidic lipids for synaptotagmin 1 function as a $\mathrm{Ca}^{2+}$ sensor. Biochem J 443, 1 (Apr 2012), 223-229.

[71] LeE, H.-K., YANG, Y., Su, Z., Hyeon, C., LeE, T.-S., LeE, H.-W., KWeON, D.-H., SHIN, Y.-K., AND YOON, T.-Y. Dynamic $\mathrm{Ca}^{2+}$-dependent stimulation of vesicle fusion by membrane-anchored synaptotagmin 1. Science 328, 5979 (May 2010), 760-763.

[72] LeUNG, K. Galactosyl serum albumin-rhodamine green. Molecular Imaging and Contrast Agent Database (MICAD): http://www.ncbi.nlm.nih.gov/books/NBK23508/, entry created: November 26, 2007 (Updated January 15, 2008).

[73] Li, L., Shin, O.-H., Rhee, J.-S., AraÇ, D., Rah, J.-C., Rizo, J., SÜDhof, T., AND Rosenmund, C. Phosphatidylinositol phosphates as co-activators of $\mathrm{Ca}^{2+}$ binding to C2 domains of synaptotagmin 1. J Biol Chem 281, 23 (Jun 2006), 1584515852.

[74] Madge, D., Elson, E., And Webb, W. Thermodynamic Fluctuations in a Reacting System - Measurement by Fluorescence Correlation Spectroscopy. Phys. Rev. Lett. 29 (1972), 705-708.

[75] Magde, D., And Elson, E. L. Fluorescence Correlation Spectroscopy. III. Uniform Translation and Laminar Flow. Biopolymers 17 (1978), 361-376.

[76] Magde, D., Elson, E. L., AND Webb, W. W. Fluorescence correlation spectroscopy. II. An experimental realization. Biopolymers 13, 1 (Jan 1974), 29-61.

[77] Malhotra, V., Orci, L., Glick, B. S., Block, M. R., and Rothman, J. E. Role of an N-ethylmaleimide-sensitive transport component in promoting fusion 
of transport vesicles with cisternae of the Golgi stack. Cell 54, 2 (Jul 1988), 221227.

[78] Martens, S., Kozlov, M. M., And McMahon, H. T. How synaptotagmin promotes membrane fusion. Science 316, 5828 (May 2007), 1205-1208.

[79] Martens, S., AND McMahon, H. T. Mechanisms of membrane fusion: disparate players and common principles. Nat Rev Mol Cell Biol 9, 7 (Jul 2008), 543-556.

[80] Marz, K. E., LAuer, J. M., And Hanson, P. I. Defining the SNARE complex binding surface of $\alpha$-SNAP: implications for SNARE complex disassembly. J Biol Chem 278, 29 (Jul 2003), 27000-27008.

[81] MAYER, A., WiCKNER, W., AND HAAS, A. Sec18p (NSF)-driven release of Sec17p ( $\alpha$-SNAP) can precede docking and fusion of yeast vacuoles. Cell 85,1 (Apr 1996), 83-94.

[82] McMahon, H. T., Kozlov, M. M., and Martens, S. Membrane curvature in synaptic vesicle fusion and beyond. Cell 140, 5 (Mar 2010), 601-605.

[83] MÜller, M., AND Davis, G. W. Vesicle priming in a SNAP. Neuron 68, 3 (Nov 2010), 324-326.

[84] Nagiec, E. E., Bernstein, A., And Whiteheart, S. W. Each domain of the N-ethylmaleimide-sensitive fusion protein contributes to its transport activity. $J$ Biol Chem 270, 49 (Dec 1995), 29182-29188.

[85] NAgY, A., BAKer, R. R., MorRis, S. J., AND WhitTAKer, V. P. The preparation and characterization of synaptic vesicles of high purity. Brain Res 109, 2 (Jun 1976), 285-309.

[86] NeHER, E. A comparison between exocytic control mechanisms in adrenal chromaffin cells and a glutamatergic synapse. Pflugers Arch 453, 3 (Dec 2006), 261268. 
[87] OtTo, H., HANSON, P. I., AND JAHN, R. Assembly and disassembly of a ternary complex of synaptobrevin, syntaxin, and SNAP-25 in the membrane of synaptic vesicles. Proc Natl Acad Sci U S A 94, 12 (Jun 1997), 6197-6201.

[88] Pace, C. N., Vajdos, F., Fee, L., Grimsley, G., And Gray, T. How to measure and predict the molar absorption coefficient of a protein. Protein Sci 4, 11 (Nov 1995), 2411-2423.

[89] Park, Y., Hernandez, J. M., van den Bogaart, G., Ahmed, S., Holt, M., RIEDEL, D., AND JAHN, R. Controlling synaptotagmin activity by electrostatic screening. Nat Struct Mol Biol (Sep 2012), http: / / dx.doi.org/10.1038/nsmb.2375.

[90] Peterson, G. L. A simplification of the protein assay method of Lowry et al. which is more generally applicable. Anal Biochem 83, 2 (Dec 1977), 346-356.

[91] Pobbati, A. V., Stein, A., And Fasshauer, D. N- to C-terminal SNARE complex assembly promotes rapid membrane fusion. Science 313, 5787 (Aug 2006), $673-676$.

[92] POHL, W. H. Entwicklung und Anwendung spektroskopischer Korrelationsverfahren zur Beantwortung biomolekularer Fragestellungen. PhD thesis, Georg-AugustUniversity Göttingen, 2009.

[93] Pohl, W. H., Hellmuth, H., Hilbert, M., Seibel, J., And Walla, P. J. A two-photon fluorescence-correlation study of lectins interacting with carbohydrated 20 nm beads. Chembiochem 7, 2 (Feb 2006), 268-274.

[94] Püschel, A. W., O'CONNOR, V., AND Betz, H. The N-ethylmaleimidesensitive fusion protein (NSF) is preferentially expressed in the nervous system. FEBS Lett 347, 1 (Jun 1994), 55-58.

[95] Radhakrishnan, A., Stein, A., Jahn, R., And Fasshauer, D. The Ca ${ }^{2+}$ affinity of synaptotagmin 1 is markedly increased by a specific interaction of its C2B domain with phosphatidylinositol 4,5-bisphosphate. J Biol Chem 284, 38 (Sep 2009), 25749-25760. 
[96] RheE, J.-S., Li, L. Y., SHIN, O.-H., RAH, J.-C., RizO, J., SüDHOF, T. C., AND RosenMund, C. Augmenting neurotransmitter release by enhancing the apparent $\mathrm{Ca}^{2+}$ affinity of synaptotagmin 1. Proc Natl Acad Sci U S A 102, 51 (Dec 2005), 18664-18669.

[97] Richards, D. A., BAI, J., AND CHAPMAN, E. R. Two modes of exocytosis at hippocampal synapses revealed by rate of FM1-43 efflux from individual vesicles. J Cell Biol 168, 6 (Mar 2005), 929-939.

[98] Rigler, R., AND ELSON, E. Fluorescence Correlation Spectroscopy: Theory and Applications. Springer, 2001.

[99] Rigler, R., Földes-Papp, Z., Meyer-Almes, F. J., SAmmet, C., VÖlCker, M., AND SCHNETZ, A. Fluorescence cross-correlation: a new concept for polymerase chain reaction. J Biotechnol 63, 2 (Aug 1998), 97-109.

[100] Rigler, R., Mets, U., Widengren, J., AND KASK, P. Fluorescence correlation spectroscopy with high count rate and low background: analysis of translational diffusion. Eur Biophys J 22 (1993), 169-175.

[101] RIZO, J., CHEN, X., AND ARAÇ, D. Unraveling the mechanisms of synaptotagmin and SNARE function in neurotransmitter release. Trends Cell Biol 16, 7 (Jul 2006), 339-350.

[102] RIZO, J., AND SÜDHOF, T. C. Snares and Munc18 in synaptic vesicle fusion. Nat Rev Neurosci 3, 8 (Aug 2002), 641-653.

[103] Rodríguez, F., Bustos, M. A., Zanetti, M. N., Ruete, M. C., Mayorga, L. S., AND TOMES, C. N. $\alpha$-SNAP prevents docking of the acrosome during sperm exocytosis because it sequesters monomeric syntaxin. PLoS One 6, 7 (2011), e21925.

[104] Schiavo, G., Gu, Q. M., Prestwich, G. D., SÖllner, T. H., And Rothman, J. E. Calcium-dependent switching of the specificity of phosphoinositide binding to synaptotagmin. Proc Natl Acad Sci U S A 93, 23 (Nov 1996), 13327-13332. 
[105] Schuette, C. G., Hatsuzawa, K., Margittai, M., Stein, A., Riedel, D., KÜster, P., KÖNIG, M., SeIdel, C., AND Jahn, R. Determinants of liposome fusion mediated by synaptic SNARE proteins. Proc Natl Acad Sci U S A 101, 9 (Mar 2004), 2858-2863.

[106] Schwartz, M. L., AND MERZ, A. J. Capture and release of partially zipped trans-SNARE complexes on intact organelles. J Cell Biol 185, 3 (May 2009), 535549.

[107] Schwille, P., Meyer-Almes, F. J., AND Rigler, R. Dual-color fluorescence cross-correlation spectroscopy for multicomponent diffusional analysis in solution. Biophys J 72, 4 (Apr 1997), 1878-1886.

[108] SCHÄGGER, H., AND VON JAGOW, G. Tricine-sodium dodecyl sulfatepolyacrylamide gel electrophoresis for the separation of proteins in the range from 1 to $100 \mathrm{kDa}$. Anal Biochem 166, 2 (Nov 1987), 368-379.

[109] Siddiqui, T. J., Vites, O., Stein, A., HeintzmanN, R., Jahn, R., ANd FASSHAUER, D. Determinants of synaptobrevin regulation in membranes. Mol Biol Cell 18, 6 (Jun 2007), 2037-2046.

[110] Steel, G. J., Buchheim, G., Edwardson, J. M., And Woodman, P. G. Evidence for interaction of the fusion protein $\alpha$-SNAP with membrane lipid. Biochem J 325 ( Pt 2) (Jul 1997), 511-518.

[111] Stein, A., RAdHAKRishnan, A., Riedel, D., FASSHAuer, D., AND JAhn, R. Synaptotagmin activates membrane fusion through a $\mathrm{Ca}^{2+}$-dependent trans interaction with phospholipids. Nat Struct Mol Biol 14, 10 (Oct 2007), 904-911.

[112] Stein, A., Weber, G., WAhl, M. C., And JAhn, R. Helical extension of the neuronal SNARE complex into the membrane. Nature 460, 7254 (Jul 2009), 525528.

[113] Sudhof, T. C. The synaptic vesicle cycle. Annu Rev Neurosci 27 (2004), 509-547. 
[114] Sutton, R. B., Fasshauer, D., Jahn, R., And Brunger, A. T. Crystal structure of a SNARE complex involved in synaptic exocytosis at 2.4 A resolution. Nature 395, 6700 (Sep 1998), 347-353.

[115] Söllner, T., Bennett, M. K., Whiteheart, S. W., Scheller, R. H., AND Rothman, J. E. A protein assembly-disassembly pathway in vitro that may correspond to sequential steps of synaptic vesicle docking, activation, and fusion. Cell 75, 3 (Nov 1993), 409-418.

[116] Sørensen, J. B., Wiederhold, K., Müller, E. M., Milosevic, I., Nagy, G., De Groot, B. L., Grubmüller, H., and Fasshauer, D. Sequential Nto C-terminal SNARE complex assembly drives priming and fusion of secretory vesicles. EMBO J 25, 5 (Mar 2006), 955-966.

[117] SüDHOF, T. C. The synaptic vesicle cycle: a cascade of protein-protein interactions. Nature 375, 6533 (Jun 1995), 645-653.

[118] Tagaya, M., Wilson, D. W., Brunner, M., Arango, N., And Rothman, J. E. Domain structure of an $\mathrm{N}$-ethylmaleimide-sensitive fusion protein involved in vesicular transport. J Biol Chem 268, 4 (Feb 1993), 2662-2666.

[119] TAKamori, S., Holt, M., Stenius, K., Lemke, E. A., Grønborg, M., Riedel, D., Urlaub, H., Schenck, S., BrÜgger, B., Ringler, P., MÜller, S. A., Rammner, B., Gräter, F., Hub, J. S., De Groot, B. L., Mieskes, G., Moriyama, Y., Klingauf, J., Grubmüller, H., Heuser, J., Wieland, F., AND JAHN, R. Molecular anatomy of a trafficking organelle. Cell 127, 4 (Nov 2006), 831-846.

[120] Tomes, C. N., De Blas, G. A., Michaut, M. A., Farré, E. V., Cherhitin, O., VISCONTI, P. E., AND MAYORGA, L. S. $\alpha$-SNAP and NSF are required in a priming step during the human sperm acrosome reaction. Mol Hum Reprod 11, 1 (Jan 2005), 43-51. 
[121] TORRES, T., AND LEVITUS, M. Measuring conformational dynamics: a new FCSFRET approach. J Phys Chem B 111, 25 (Jun 2007), 7392-7400.

[122] VAn Der MeER, W., COKer, G., AND Chen, S.-Y. Resonance Energy Transfer: Theory and Data. Wiley VCH, 1994.

[123] Vennekate, W., Schröder, S., Lin, C.-C., VAn den BogaArt, G., GrunWALD, M., JAHN, R., AND WALLA, P. J. Cis- and trans-membrane interactions of synaptotagmin-1. Proc Natl Acad Sci U S A 109, 27 (Jul 2012), 11037-11042.

[124] Verstreken, P., Ohyama, T., And Bellen, H. J. FM 1-43 labeling of synaptic vesicle pools at the Drosophila neuromuscular junction. Methods Mol Biol 440 (2008), 349-369.

[125] Vrljic, M., Strop, P., Ernst, J. A., Sutton, R. B., Chu, S., And Brunger, A. T. Molecular mechanism of the synaptotagmin-SNARE interaction in $\mathrm{Ca}^{2+}$ triggered vesicle fusion. Nat Struct Mol Biol 17, 3 (Mar 2010), 325-331.

[126] Walter, A. M., Wiederhold, K., Bruns, D., Fasshauer, D., AND SøRENSEN, J. B. Synaptobrevin N-terminally bound to syntaxin-SNAP-25 defines the primed vesicle state in regulated exocytosis. J Cell Biol 188, 3 (Feb 2010), 401-413.

[127] WANG, L., Ungermann, C., AND WiCKNER, W. The docking of primed vacuoles can be reversibly arrested by excess Sec17p ( $\alpha$-SNAP). J Biol Chem 275, 30 (Jul 2000), 22862-22867.

[128] Weber, T., Zemelman, B. V., McNew, J. A., Westermann, B., Gmachl, M., PARlati, F., SÖllner, T. H., And Rothman, J. E. SNAREpins: minimal machinery for membrane fusion. Cell 92, 6 (Mar 1998), 759-772.

[129] Widengren, J., Schweinberger, E., Berger, S., And SEidel, C. Two New Concepts to Measure Fluorescence Resonance Energy Transfer via Fluorescence Correlation Spectroscopy: Theory and Experimental Realizations. The Journal of Physical Chemistry A 105, 28 (2001), 6851-6866. 
[130] Widengren, J., AND SChwille, P. Characterization of Photoinduced Isomerization and Back-Isomerization of the Cyanine Dye Cy5 by Fluorescence Correlation Spectroscopy. The Journal of Physical Chemistry A 104, 27 (2000), 6416-6428.

[131] Wimmer, C., Hohl, T. M., Hughes, C. A., Müller, S. A., Söllner, T. H., ENGel, A., AND Rothman, J. E. Molecular mass, stoichiometry, and assembly of 20 S particles. J Biol Chem 276, 31 (Aug 2001), 29091-29097.

[132] Winter, U., Chen, X., AND Fasshauer, D. A conserved membrane attachment site in $\alpha$-SNAP facilitates $\mathrm{N}$-ethylmaleimide-sensitive factor (NSF)-driven SNARE complex disassembly. J Biol Chem 284, 46 (Nov 2009), 31817-31826.

[133] XU, T., Ashery, U., Burgoyne, R. D., AND NeHer, E. Early requirement for $\alpha$-SNAP and NSF in the secretory cascade in chromaffin cells. EMBO J 18, 12 (Jun 1999), 3293-3304.

[134] Xue, M., Ma, C., Craig, T. K., Rosenmund, C., And Rizo, J. The Janusfaced nature of the $\mathrm{C}(2) \mathrm{B}$ domain is fundamental for synaptotagmin-1 function. Nat Struct Mol Biol 15, 11 (Nov 2008), 1160-1168.

[135] YANG, X., Kaeser-WoO, Y. J., PANG, Z. P., XU, W., AND SÜDHOF, T. C. Complexin clamps asynchronous release by blocking a secondary $\mathrm{Ca}^{2+}$ sensor via its accessory $\alpha$ helix. Neuron 68, 5 (Dec 2010), 907-920.

[136] YU, R. C., JAHN, R., AND BRUnger, A. T. NSF N-terminal domain crystal structure: models of NSF function. Mol Cell 4, 1 (Jul 1999), 97-107.

[137] Zhao, C., SMith, E. C., AND Whiteheart, S. W. Requirements for the catalytic cycle of the N-ethylmaleimide-Sensitive Factor (NSF). Biochim Biophys Acta 1823, 1 (Jan 2012), 159-171.

[138] van den BogaArt, G., Holt, M. G., Bunt, G., Riedel, D., Wouters, F. S., AND JAHN, R. One SNARE complex is sufficient for membrane fusion. Nat Struct Mol Biol 17, 3 (Mar 2010), 358-364. 
[139] VAN DEN BOGAART, G., AND JAHN, R. Inside insight to membrane fusion. Proc Natl Acad Sci U S A 108, 29 (Jul 2011), 11729-11730.

[140] Van den BogaArt, G., Meyenberg, K., Diederichsen, U., And Jahn, R. Phosphatidylinositol 4,5-bisphosphate increases $\mathrm{Ca}^{2+}$ affinity of synaptotagmin1 by 40-fold. J Biol Chem 287, 20 (May 2012), 16447-16453.

[141] Van den BogaArt, G., Thutupalli, S., Risselada, J. H., MeyenberG, K., Holt, M., Riedel, D., Diederichsen, U., Herminghaus, S., GrubMÜller, H., AND JAHN, R. Synaptotagmin-1 may be a distance regulator acting upstream of SNARE nucleation. Nat Struct Mol Biol 18, 7 (Jul 2011), 805-812. 


\section{List of Figures}

1.1 Synaptic vesicle cycle. . . . . . . . . . . . . . . . . . . 2

1.2 Four-helix-bundle of the core SNARE complex. . . . . . . . . . . 3

1.3 Layer structure of the ternary SNARE complex. . . . . . . . . . . . 4

1.4 The SNARE conformational cycle during vesicle docking and fusion. . . 5

$1.5 \quad$ 3D structure of synaptotagmin-1. $\ldots \ldots \ldots \ldots \ldots \ldots \ldots$

1.6 Membrane attachment site of $\alpha$-SNAP in the presence of the SNARE complex. . . . . . . . . . . . . . . . . . . . . . . 10

1.7 Fluorescence autocorrelation spectroscopy. $\ldots \ldots \ldots \ldots \ldots \ldots$

$1.8 \quad$ Fluorescence cross-correlation-spectroscopy. $\ldots \ldots \ldots \ldots$. . . . . 15

1.9 OPE compared with TPE in terms of the excitation module and the effective volume. . . . . . . . . . . . . . . . . . . 17

1.10 Jablonski-diagram of FRET donor and acceptor. $\ldots \ldots \ldots \ldots$

1.11 Excitation and emission spectra of TR-DHPE and OG-DHPE. . . . . . . 19

$2.1 \quad$ Expression test of Syt1 under different conditions. . . . . . . . . . . . 31

2.2 Purification of Syt1 with immobilized metal ion affinity chromatography and ion exchange chromatography. . . . . . . . . . . . . . 33

$2.3 \quad$ Size exclusion chromatography using sephadex G50 econo column to reconstitute small liposomes. . . . . . . . . . . . . . . . . . . 37 
2.4 Confocal fluorescence microscopy setup for two-photon excitation. . . . 41

3.1 Schematic of typical FCCS curves in the tethering assay. . . . . . . . . . . 44

3.2 Example FCCS data from the tethering experiments. . . . . . . . . . . . . 45

3.3 Domain structures of Syt1 and its mutants used in this experiment. . . . 46

$3.4 \quad$ Liposome tethering mediated by membrane-anchored Syt1. . . . . . . . 48

3.5 Syt1 mediated membrane tethering in the presence of $12 \%$ and of $5 \%$ PS in the cis-membrane. . . . . . . . . . . . . . . . . . . . 50

3.6 Domain structures of the soluble C2AB domain and its Alexa Fluor 488 labeled mutant. . . . . . . . . . . . . . . . . . . . . . 50

3.7 Clustering of liposomes mediated by soluble C2AB domain. . . . . . . . 51

$3.8 \quad$ Binding assay of soluble C2AB labeled with alexa fluor 488 to TRPS liposome containing $20 \%$ PS. . . . . . . . . . . . . . . 52

3.9 Binding kinetics between liposomes (A) and AF-C2AB (B). . . . . . . . . 54

3.10 Tethering of liposomes mediated by membrane-anchored Syt1-SNAREs interactions. ....................... 57

3.11 Molecular model of an average SV. . . . . . . . . . . . . . . 58

3.12 SV labeling with FM 1-43 dye. . . . . . . . . . . . . . . . . . 60

3.13 FCS measurement of rat SVs in three dilutions compared to FM 1-43 background. ........................... 61

3.14 SV concentration plotted versus the protein concentration of the same sample. . . . . . . . . . . . . . . . . . 63

3.15 Calculated protein mass per SV for mouse compared to rat. . . . . . . . . 64

3.16 Schmatic of CG docking with $100 \mathrm{~nm}$ liposomes in the presence of $\alpha$-SNAP. 66

3.17 Diffusion time determination of the liposome docking experiments with CGs using FCS. . . . . . . . . . . . . . . . . . . 68 
3.18 Histogram of the docking experiment using liposome and CG. . . . . . . 69

$3.19 \alpha$-SNAP does not block the SNARE-mediated CG docking. . . . . . . . . 71

$4.1 \quad$ Model of the trans-tethering of the membrane anchored Syt1 to the target membrane containing either acidic phospholipids or SNAREs. . . . . . . 74

4.2 All trans-tethering is inhibited if the host liposome of Syt1 contains 20\% PS. 75

$4.3 \mathrm{C} 2 \mathrm{AB}$ fragment is able to cluster the liposome under a saturating concentration on the liposome surface. . . . . . . . . . . . . . . . 75 


\section{List of Tables}

2.1 Phospholipids used for reconstitution of liposomes. . . . . . . . . . . . . 22

2.2 Proteins used in this study . . . . . . . . . . . . . . . . . . . 24

2.3 Buffers used in this study $\ldots \ldots \ldots \ldots \ldots \ldots$

2.4 Instruments, filters, columns and miscellanea. . . . . . . . . . . . . . 29

2.5 Tricine (Schägger)-gels. . . . . . . . . . . . . . . . . 36

2.6 Lipid composition of the liposomes in the Syt1 tethering experiments. . 38

$3.1 \quad$ Binding constant between liposomes and AF-C2AB fragments. . . . . . . 53 


\section{Abbreviations and Symbols}

$\alpha$-SNAP

$A b s$

aa

AAA

APS

BSA

CG

CHAPS

Cys

$\mathrm{ddH}_{2} \mathrm{O}$

DNAse I

DOC

DTT

EDTA

f.c.

$\mathrm{fl}$
$N$-Ethylmaleimide-sensitive factor attachment protein, $\alpha$

Absorption

Amino acids

ATPases associated with a variety of cellular activities

Ammonium persulfate

Bovine serum albumin

Chromaffin granules

3-[(3-Cholamidopropyl)dimethylammonio]-1-propanesulfonate

Cysteine

Double distilled water

Deoxyribonuclease I

Deoxycholic acid

Dithiothreitol

Ethylenediaminetetraacetic acid

Final concentration

Femtoliter 
FRET

h

HEPES

IEXC

IMAC

IPTG

$\mathrm{KCl}$

LUVs

Milli-Q

$\min$

NA

NBD-DOPE

Ni-NTA

NSF

OD

OGPE

OPE

P:L

PC

PE
Förster Resonance Energy Transfer

Hours

4-(2-Hydroxyethyl)piperazine-1-ethanesulfonic acid

Ion Exchange Chromatography

Immobilized Metal ion Affinity Chromatography

Isopropyl- $\beta$-D-thiogalactopyranosid

Potassium chloride

Large unilamellar vesicles

Ultrapure water

Minutes

Numerical aperture

1,2-Dioleoyl-sn-glycero-3-phosphoethanolamine- $N$-(7-nitrobenz2-oxa-1,3-diazol-4-yl)

$\mathrm{Ni}^{2+}$-nitrilotriacetic acid beads

$\mathrm{N}$-Ethylmaleimide-sensitive factor

Optical density

Oregon Green-PE

One-photon excitation

Molar protein to lipid ratio

L- $\alpha$-Phosphatidylcholine

L- $\alpha$-Phosphatidylethanolamine 
PI

PiP2

PMSF

PS

RG

Rhodamine-DOPE

RPM

RT

SDS

SDS-PAGE

SEM

SNAP25A

SNAPs

SNARE

Sx1A

Syt1

TCA

TEMED

TPE

Tricine
L- $\alpha$-Phosphatidylinositol

Phosphatidylinositol-4,5-bisphosphate

Phenylmethanesulfonylfluoride

L- $\alpha$-Phosphatidylserine

Rhodamine Green

1,2-dioleoyl-sn-glycero-3-phosphoethanolamine- $N$-lissamine rhodamine B sulfonyl ammonium salt

Revolutions per minute

Room temperature

Sodium dodecyl sulfate

Sodium dodecyl sulfate polyacrylamide gel electrophoresis

Standard error of the mean

Synaptosomal-associated protein $25 \mathrm{~A}$

Soluble NSF attachment proteins

Soluble $N$-ethylmaleimide-sensitive-factor attachment receptor

Syntaxin $1 \mathrm{~A}$

Synaptotagmin-1

Trichloroacetic acid

$N, N, N^{\prime}, N^{\prime}$-Tetramethylethylendiamine

Two-photon excitation

$N$-(Tri(hydroxymethyl)methyl)glycine 
Trp Tryptophan

TRPE

Texas Red-PE

Tyr

Tyrosine

$\mathrm{v} / \mathrm{v}$

volume/volume

$\mathrm{w} / \mathrm{v}$

weight/volume 


\section{Publication}

Vennekate W, Schröder S, Lin CC, van den Bogaart G, Grunwald M, Jahn R, Walla PJ. "Cis- and trans-membrane interactions of synaptotagmin-1." Proc. Natl. Acad. Sci. USA 109, 11037-11042 (2012).

Another manuscript is in preparation. 


\section{Acknowledgement}

I would like to thank both of my supervisors Professor Peter Jomo Walla and Professor Reinhard Jahn, for supporting me to complete my PhD thesis with many pleasant discussions and many important suggestions. Also I would like to thank Professor Claudia Steinem for joining my thesis committee and giving me lots of useful suggestions.

My collaboration partners, Dr. Geert van den Bogaart, Dr. Yongsoo Park, Dr. Saheeb Ahmed and Dr. Matthew Holt, have to be named not only for offering an active working atmosphere, but also for the growing friendships with them during the past four years.

I would like to give special thanks to Dr. Anna Cypionka and Dr. Wiebke Hanna Pohl for getting me start in the work group and sharing a great time with me.

I would like to thank all my friends of the Walla group and the Jahn department for having an enjoyable time with them. I thank for the great help from Dr. Javier Matias Hernandez, Jianhua Chen, Matthias Grunwald, Sabrina Schröder, Chao-Chen Lin and Mrs. Ursel Ries during my pregnancy leave, which enabled me to continue my project during an important period.

Finally, I would like to thank Inge Dreger and Hendrik Vennekate for revising the manuscript. 


\section{Curriculum Vitae}

\section{Personal information}

Name: Wensi Vennekate, née Gao

Date of birth: December 23rd, 1982

City of birth: Tongliaoshi, China

Nationality: German

\section{Education}

2009/01-present Ph.D. student in Chemistry in the workgroup Biomolecular Spectroscopy and Single-Molecule Detection, Max-Planck-Institute for Biophysical Chemistry, Göttingen, Germany.

2008/11-2008/12 Practical training in the workgroup Biomolecular Spectroscopy and Single-Molecule Detection, Max-Planck-Institute for Biophysical Chemistry, Göttingen, Germany.

2003/10-2008/10 Diploma in Chemistry. Georg-August-University, Göttingen, Germany.

Thesis Title: Wechselwirkung neuer Cholinkinase-Inhibiroren mit den Enzymen von Malaria-Plasmodium (Interaction of Novel Cholinkinase Inhibitors with Malaria Plasmodium).

2002/12-2003/09 German language course (DSH), Goethe-Institute, Göttingen, Germany.

2001/09-2002/08 Student of industrial design, Beijing University of Technology, China.

1998/09-2001/08 Beijing No. 4 High School, China. 